UC-20d

Issued: March 1987

LA--10908-MS

DE87 007724

\title{
The Spheromak as a Compact Fusion Reactor
}

\author{
R. L. Hagenson* \\ R. A. Krakowski
}

\section{DISCLAIMER}

\begin{abstract}
This report was prepared as an account of work sponsored by an agency of the United States Government. Neither the United States Government nor any agency thereof, nor any of their employees, makes any warranty, express or inplied, or assumes any legal liability or responsibility for the accuracy, complcteness, or usefulness of any information, apparatus, product, or process disclosed, or represents that its use would not infringe privately owned rights. Reference herein to any specific commercial product, process, or service by trade name, trademark, manufactuier, or otherwise does not necessarily constitute or imply its endorsement, recommendation, or favoring by the United States Government or any agency thereof. The views and opinions of authors expressed herein do not necessarily state or reflect those of the United States Government or any agency thereof.
\end{abstract}


Abstract . . . . . . . . . . . . . . . . . . . . . . 1

1. Introduction . . . . . . . . . . . . . . . . . . . . . . 1

1.1. Background and Rationale for Compact Fusion Reactors . . . . . . 2

1.1.1. Cost-Based Issues . . . . . . . . . . . . . . . . . . . . 3

1.1.2. Utility-Based Issues for Improved Fusion Reactors . . . . 13

1.2. Approaches to Improved Fusion Reactors . . . . . . . . . . . . . 15

1.3. Overview of Spheromak Concept . . . . . . . . . . . . . . . . . . 18

2. Study Background and Approach . . . . . . . . . . . . . . . . . . . 21

2.1. Background . . . . . . . . . . . . . . . . . . . . . 21

2.2. Approach . . . . . . . . . . . . . . . . . . . . . 31

2.2.1. Overview . . . . . . . . . . . . . . . . 31

2.2.2. Physics..................... . 33

2.2.3. Magnetics... . . . . . . . . . . . . . . 36

2.2.4. Engineering . . . . . . . . . . . . . . . . . . . 37

2.2.5. Economics . . . . . . . . . . . . . . . . . 37

2.2.6. Systems . . . . . . . . . . . . . . . . . . . . . 38

3. Parametric Systems Model . . . . . . . . . . . . . . . . . . 45

3.1. Equilibrium Magnetics . . . . . . . . . . . . . . . . . 45

3.2. Plasma Model . . . . . . . . . . . . . . . . . . . 50

3.3. Current-Drive (Electrode/Gun) System . . . . . . . . . . . . 53

3.4. Engineering Power Balance . . . . . . . . . . . . . . . 60

3.5. Economics . . . . . . . . . . . . . . . . . 66

4. Results . . . . . . . . . . . . . . . . . . . . 71

4.1. Basecase Parameters . . . . . . . . . . . . . . . 71

4.2. Major Variable Sensitivities . . . . . . . . . . . . . 76

4.3. Preliminary Consideration for Fusion-Power-Core Layout . . . . . 78

5. Summary and Conclusions . . . . . . . . . . . . . . . . . . . 91

5.1. Design Summary and Comparisons . . . . . . . . . . . . . . 91

5.2. Conclusions . . . . . . . . . . . . . . . . . . . . 92 Acknowledgments . . . . . . . . . . . . . . . . . . . . . 97 Nomenclature . . . . . . . . . . . . . . . . . . . . . 99 References . . . . . . . . . . . . . . . . . . . . . 107 Appendix A: Estimate of Heat Loss From a "Cylindrical" Spheromak . . . . 115 
THE SPHEROMLK AS A COMPACT FUSION REACTOR

by

R. L. Hagenson and R. A. Krakowski

\title{
ABSTRACT
}

\begin{abstract}
After sumarizing the economic and utility-based rationale for compact, higher-pover-density fusion reactors, the gun-sustained spheromak concept is explored as one of a number of poloidal-field-dominated confinement configurations that might improve the prospects for economically attractive and operationally simplified fusion power plants. Using a comprehensive physics/engineering/costing model for the spheromak, guided by realistic engineering constraints and physics extrapolation, a range of cost-optimized reactor design points is presented, and the sensitivity of cost to key physics, engineering, and operational variables is reported. The results presented herein provide the basis for conceptual engineering designs of key fusion-power-core (FPC) subsystems and more detailed plasma modeling of this promising, high mass-power-density concept, which stresses single-piece FPC maintenance, steady-state current drive through electrostatic magnetic helicity injection, a simplified co-axial electrode-divertor, and efficient resistive-coil equilibrium-field coils. The optimal FPC size and cost estimates project a system that competes aggressively with the best offered by alternative energy sources while simplifying considerably the complexity that has generally been associated with most approaches to magnetic fusion energy.
\end{abstract}

\section{INTRODUCTION}

The results of a preliminary but comprehensive study of the commercial reactor potential of the spheromak Compact Torus (CT) are reported. The emphasis of this study is the compact, high-power-density approach which attempts to minimize the impact of the fusion power core (FPC) (i.e., the plasma chamber, first wall, blanket, shield, impurity control, current drive, confining coils, and associated structure) on the overall cost of fusion power. This introduction presents a general background and rationale for the compact, high- 
power-density approach (Sec. 1.1.), a range of approaches to such improved fusion schemes (Sec. 1.2.), and a brief overview of the spheromak concept (Sec. 1.3.). Section 2. gives the background for and an approach to the study of the Compact Spheromak Reactor (CSR), along with the details of the physics, engineering, and costing models that provide the basis of the parametric syatems model being given in Sec. 3. Parametric results and comparisons of costoptimized fusion reactors are summarized in Sec. 4., and Sec. 5. gives a summary of and conclusions from this preliminary scoping study of the CSR.

\subsection{Background and Rationale for Compact Fusion Reactors}

Ideally, a new energy source must demonstrate a capability of displacing old energy sources while providing both economic opportunities and enhanced environmental benefits. The attraction of an essentially unlimited fuel supply has generated the impetus to develop advanced fission breeders and, even more strongly, to exploit nuclear fusion. Both fission and fusion systems trade off a reduced fuel cost with a more capital-intensive plant needed to utilize a cheaper and more abundant fuel. Results from early conceptual designs of fusion power plants,1-10 however, indicaced that these systems could be so capital intensive as to override any inherent cost savings promised by an inexpensive fuel cycle. Early warnings of these problems appeared,11-13 but until recently specific solutions to this growing concern were few. Generalized rcutas have recently been suggested by which fusion could be made more economically attractive.14,15 Specific examples for improved fusion reactors alsc have recently been reported.16,17

The generally recognized problems of large size, technological complexiıy, and correspondingly high cost of a magnetic fusion power plant strorgly suggest directions of improvement. Although a reduction in the nass (and cost) of the FPC most directly reduces the cost of fusion power, with the mass power censity (MPD, ratio of net electric power to FPC mass, kWe/tonne) hing suggested as a good figure-of-merit in this respect, ${ }^{18}$ other technicai, safety/environin.ntal, and institutional issues also enter into the definition and dicecticn of improved fusion concepts. 
1.1.1. Cost-Based Issues. The large FPCs projected fo: sasly conceptual reactor designs are reflected in a high capital cost, usually expressed as a unit direct cost, UDC(\$/kWe), and a high cost of electricity, $\operatorname{coE}(\mathrm{mills} / \mathrm{kWeh})$. These $:=t$ estimates contain uncertainties both in the level of physical performan zeauired of the plasma and in the cost of individual FPC components that support that plasma. Investment cost or UDC uncertainties of 20-25\% have been estinated, with failure to achieve the design net power, plasma power density, neutron vall loading, and materials performance having dramatic influences on the calculated UDC and $\operatorname{COE} ;^{19}$ depending on the plant availability factor, $P_{f}$, the uncertainty in COE can exceed the uncertainty associated with the UDC. Although difficult to quantify at the present stage of fusion development, if the fusion-power-core MPD can be made sufficiently high, the impact of the FPC on $p_{f}$ can be reduced considerably through the adaptation of few- or single-piece-inaintenance procedures. On the other hand, however, extended FPC lifetime can be achieved in systems with larger, lower-MPD fusion power cores, although maintenance and repair of such systems may be unwieldy and require extensive downtime. Clearly, a tradeoff exists between the mean time to fail and the mean time to repair through both UDC and $p_{f}\left(C O E \propto U D C / p_{f}\right)$, and the required optimization will play an important part in the overall cost equation for fusion. With $p_{f}$ being nominally fixed or weakly dependent on power density, both UDC and COE remain as the most useful figures-of-merit by which to intercompare fusion concepts as well as for comparing fusion with alternative energy sources.

The impact of physics and technology on the cost of fusion power is illustrated graphically on Fig. 1.1.-1, which shows the major elements and influences that determine the UDC and COE. Figure 1.1.-1 is organized according to the cost accounting system used in the fission-power industry ${ }^{2}$ and adopted to characterize fusion-power systems.21,22 The major components of the total direct cost are conveniently divided into two major cost categories: Reactor Plant Equipment, RPE (Account 22. in Refs. 20-22, with the FPC being Account 22.1.1. under the Reactor Equipment, Account 22.1.) and the Balance of Plant (BOP, Accounts 21., 23., 24., 25., and 26., in the order listed in Fig. 1.1.-1). Fnr fusion power plants invoking more-or-less conventional BOPs (i.e., steambased conversion systems with gross conversion efficiencies of $\eta_{\mathrm{TH}}=0.35-0.40$ ), the RPE for the early fusion power plant designs ${ }^{-10}$ alone represented $\geq 50 \%$ of the total direct cost, with the FPC requiring $25-30 \%$ of all direct expenditures; 


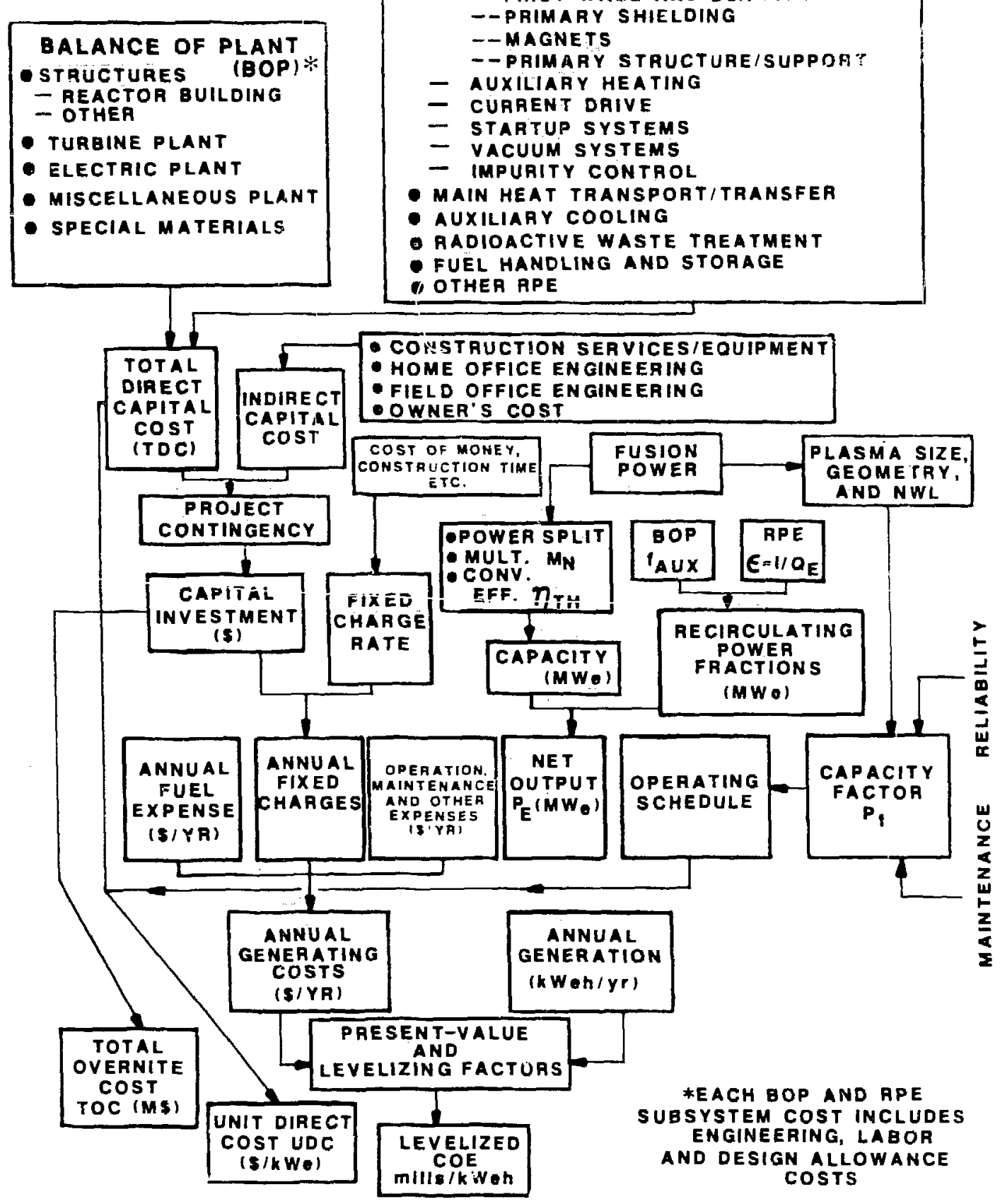

Reactor equifment

- fusion power core iffCi

- - FIRST WALL AND BLAF:

- PRIMARY SHIELOING

- MAGNETS

- - PRIMARY STRUCTURE/SUPPOAT

- AUXILIARY HEATING

- CURAENT DRIVE

- STARTUP SYSTEMS

- VACUUM SYSTEMS

IMPUAITY CONTAOL

WIAAY COOLING

RADIOACTIVE WASTE TREATMENT

FUEL HANDLING AND STORAGE

OTHER APE

Fig. 1.1.-1. Essential elements determining the cost of a fusion power plant as well as the major componants of the Reactor Plant Equipment (RPE, Account 22.) and the Balance of Plant (BOP). 
these percentages compare to $\sim 30 \%$ and $\leq 5 \%$, respectively, for identical accounts in a typical light-water fission reactor (LWR).23 Table 1.1.-I summarizes the major costs for a number of earlier fusion power-plant designs, as well as recently improved designs based either on innovative approaches to the tokamak ${ }^{4}$ or extensions from non-tokamak concepts ${ }^{2-27}$; a normalized comparison to the pressurized-water fission reactor, $\mathrm{PWR}^{23}$, is also included. Both the magnitude of and sensitivity to the RPE and (particularly) the FPC costs, as well as required (extrapolated) physics and materials performances related thereto, point to a key area where the economic prospects for fusion can be increased and the associated time and risks required for commercialization can be decreased: increased FPC power density and decreased FPC size (mass, cost, and complexity). This approach to an improved fusion reactor, however, is not without the technical and economic compromises illustrated on Fig. 1.1.-1 and discussed later. Specifically, increased MPD will have implications for safety (i.e., nuclear afterheat power density and degree of inherent safety ${ }^{29}$ ), environmental impact (character and quantity of radioactive waste), plasma performance [efficiencies of plasma energy confinement, $X_{E} \simeq r_{p}{ }^{2 / 4 \tau_{E}}$, and magnetic-field utilization, $\beta$, with $\left.\beta / X_{E}=3.11(B a)^{2} /\left(n \tau_{E} T / 10^{20}\right)\right]$, development cost and flexibility (both time and funding), as well as end-product cost (i.e., UDC and COE).

The target goal for acceptable COE values was achieved by increasing the MPD as more realism entered into the reactor design evolution shown on Table 1.1.-I. At the risk of oversimplifying the complex and interrelated interactions illustrated on Fig. 1.1.-1 for the COE determination, a simplified model for COE is described to show the relationships between COE, unit costs, and. MPD. If $\mathrm{f}_{1}$ is the fraction of the plant direct cost given to indirect charges, interest during construction, and escalation during construction, and if $\mathrm{f}_{2}$ is the fraction of annual charges given to operating and maintenance, spare parts, and fuel charges, then the COE can be simply expressed as follows in terms of an effective payout rate, $\lambda^{\prime} \equiv \lambda\left(1+\mathrm{f}_{1}\right)\left(1+\mathrm{f}_{2}\right) / \mathrm{p}_{\mathrm{f}}$, and the UDC: 
TABLE $1.1 .-1$

COMPARISON OF CONSTANT-DULLAR COSTS NORMALIZED AS PERCENTACE OF

TOTAL DIRECT COST FOR A RANGE OF CONCEPTUAL FUSION REACTORS

(1980 DOLLARS, a factor 1.348 takes these costs to 1986)

$\underline{\text { ACCOUNT }}$

20. Land and Land Rights

21. Structure and Site Facilities

22. Reactor Plant Equipment (RPE)

22.1.1. First Wall/Blanket

22.1.2. ShieId

22.1.3. Colls

FPC

23. Turbine Plant Equipment

24. Electric Plant Equipment

25. Hisc. Plant Equipment

26. Spectal Materials

90. Total Direct Costs (TDC)

99. Total Costs

Unit Direct Cost, UDC( $/ \mathrm{kVe})$

Cost of Elec., $\operatorname{CoE}(m i l l s / k$ Weh $)$

Unit FPC costs, $c_{F P C}(\$ / \mathrm{kg})$

Net Electric Power, $P_{E}$ (MWe)
UUMAK-I ${ }^{1}, 19$ STARFIRE

MARS $^{10}$

CRFPK $^{25,26}$

ATR/ST ${ }^{2}$

$\underline{\mathrm{CSR}}^{27} \quad \underline{\mathrm{PRH}}^{3}$

$\begin{array}{ccccccc}0.11 & 0.19 & 0.21 & 0.30 & 0.22 & 0.34 & -- \\ 13.11 & 20.09 & 10.56 & 24.41 & 18.58 & 27.00 & 22.34 \\ 53.82 & 56.00 & 64.15 & 37.31 & 51.29 & 30.43 & 34.01 \\ 6.95 & 4.77 & 3.01 & 0.95 & 2.98 & 1.90 & -- \\ 3.88 & 10.78 & 3.17 & 0.19 & 1.12 & -- & -- \\ 17.82 & 9.90 & 20.84 & 3.09 & 11.03 & 2.78 & - \\ 28.65 & 25.48 & 27.02 & 4.23 & 15.13 & 4.68 & -5 .-6 . \text { (c) } \\ 16.01 & 14.47 & 11.63 & 20.17 & 16.16 & 22.81 & 24.99 \\ 13.40 & 6.77 & 6.76 & 10.17 & 8.01 & 12.41 & 8.56 \\ 0.88 & 2.37 & 1.40 & 3.76 & 2.82 & 4.28 & 4.67 \\ 2.65 & 0.014 & 5.28 & 3.89 & 2.91 & 2.72 & 5.33 \\ 100 . & 100 . & 100 . & 100 . & 100 . & 100 . & 100 . \\ 154.25 & 185.23 & 138.10 & 136.28 & 138.87 & 136.28 & 158.93 \\ 150 . \text { (a) } & 1439 . & 1633 .(\text { b) } & 1112 . & 1485 . & 977.8 & 562 . \text { (d) } \\ 36.1 \text { (a) } & 35.1 & 38.3 \text { (b) } & 27.9 & 37.8 & 24.6 & - \\ 7 . & 19 . & 25 . & 42 . & 45 . & 53 . & 40-50 \\ 137 . & 1200 . & 1202 . & 1000 . & 1000 . & 1000 . & 1139 .\end{array}$

(6)Originally reported as $742 \$ / \mathrm{kWe}$ and $23.3 \mathrm{mills} / \mathrm{kWeh}$ in 1974 dollars; a factor of 1.55 converts to common-base $1980 \cos t s .20$

(b) Based on 1966 \$/kUe and $46 \mathrm{mills} / \mathrm{kHeh}$ in 1983 dollars; factor of 1.21 converts back to common-base $1980 \cos t s .21$

(c) Not explicitly reported in Ref. 23. but the $\sim 1000$-tonne pressure vessel (including heads) costed at - $50 \$ / \mathrm{kg}$ vould give the listed value. Reference 23 reports the Nuclear Steam Supply System (NSSS) cost is $20.49 x$ of the total direct $\cos t$.

(d)Originally reported in 1984 dollars, a factor of 1.25 converts to common-base 1980 costs.20 


$$
\begin{aligned}
\cdot C O E & \equiv \frac{A C+(O \& M+S C R+F)(1+E)^{P}}{8760 P_{E} P_{f}} \\
& =\lambda\left(1+f_{1}\right)\left(1+f_{2}\right)(U D C) / 8.76 p_{f} \\
& =\left(\lambda^{\prime} / 8.76\right)(\text { UDC }),
\end{aligned}
$$

where $p_{f}$ is the "plant factor" or fraction of the year that electrical power is generated at full capacity, $P_{E}$, and $\lambda(1 / y r)$ is the fixed charge rate. In the first statement of $\mathrm{COE}$ in Eq. (1.1.-1), AC is the annual investment charge, 0\&M is the annual operating and maintenance charge, SCR is the annual sparecomponent replacement charge, $\mathrm{E}$ is the escalation rate, and $\mathrm{P}(\mathrm{yr})$ is the construction period. Table 1.1.-II gives typical values for these economic parameters used to estimate the cost of both fusion and fission systems. For a plant factor of $p_{f}=0.75$, the effective pay rate, $\lambda^{\prime}$, equals $0.37 \mathrm{yr}^{-1}$, or the annual revenue generated by every unit of power installed must equal $37 \%$ of the initial cost to install that unic. It is for these reasons that reduced values of UDC are desirable. For many of the early fusion reactor designs, ${ }^{-10}$ the FPC and associated RPE items are major contributors to the direct cost (Table 1.1.-I) and, therefore, are areas where important reductions in the cost of fusion power can be made.

The FPC characteristics are related to the COE primarily through the plant factor, construction time (e.g., $\mathrm{f}_{1}, \mathrm{f}_{2}$ ), and most explicitly through the UDC. The unit direct cost can be approximated by the following simplified expression:

$$
\mathrm{UDC}(\$ / \mathrm{kWe})=\mathrm{FC}+10^{3} \mathrm{c}_{\mathrm{FPC}} / \mathrm{MPD}
$$

where the "fixed" costs, FC, are relatively independent of FPC and are generally a function of the level of electric power, thermal power, and recirculating power; the second term, $c_{F P C}(\$ / \mathrm{kg})$, gives the unit cost of the FPC, typical values of which are given on Table 1.1.-I for a range of conceptual reactor designs. For a fusion power plant of thermal-conversion efficiency $\eta_{T H}$, recirculating power fraction $\varepsilon$, and blanket neutron-energy multiplication $M_{N}$, the mass power density can be expressed in terms of the fusion-neutron wall loading, $I_{W}\left(M W / \mathrm{m}^{2}\right)$, as follows: 


\section{ECONOMIC PARAMETERS TYPICALLY USED IN COSTING FISSION}

\section{AND FUSION POWER PLANTS}

Fixed charge rate, $\lambda(1 / y r)$

0.15

Construction period (yr)

5 .

Ratio of indirect to direct costs

0.25

Ratio of interest-during-construction to direct costs

0.15

Ratio of escalation-during-construction to direct costs

0.0

(constant dollars)

Sum of last three fractions, $\mathrm{f}_{1}$

0.50

Ratio of annual operating/maintenance to annual charges

0.20

Ratio of annual spare-parts charges to annual charges

0.05

Ratio of annual fuel charges to annual charges

0.02

Sum of last three fractions, $\mathrm{f}_{2}$

0.27

Effective charge rate, $\lambda\left(1+f_{1}\right)\left(1+f_{2}\right)(1 / y r)$

Sample reactor conditions

- plant factor, $p_{\mathrm{f}}$

0.75

- effective pay rate, $\lambda^{\prime}=\lambda\left(1+f_{1}\right)\left(1+f_{2}\right) / p_{f}(1 / y r)$

0.36

- FPC/total cost goal

- thermal conversion efficiency, $n_{\mathrm{TH}}$

0.35

- recirculating power fraction, \&.

0.1 .5

- blanket energy multiplication, $M_{N}$

1.3

- thickness of FPC, $\Delta(\mathrm{m})$

1.5

- average FPC mass density, $\rho_{F P C}\left(\right.$ tonne $\left./ \mathrm{m}^{3}\right)$

3.

- average unit cost of FPC, $c_{F P C}(\$ / \mathrm{kg})$

$\geq 30$.

- self-consistent values

- unit direct cost, UDC(\$/kWe)

$\geq 1000$.

- mass power density, MPD(kWe/tonne)

$\geq 300$.

- neutron wall loading, $I_{W}\left(\mathrm{MW} / \mathrm{m}^{2}\right)$

$\geq 5.9 \mathrm{MW} / \mathrm{m}^{2}$

- cost of electricity, coE(mills/kWeh) 


$$
\operatorname{MPD}(k W e / \text { tonne })=\frac{1000 n_{\mathrm{TH}}(1-\varepsilon)\left(M_{\mathrm{N}}+1 / 4\right)}{2 \rho_{\mathrm{FPC}}{ }^{\Delta}} I_{W} \text {, }
$$

where the average ("smeared") mass density of the FPC is $\rho_{F P C}\left(\right.$ tonne/ $\left./ \mathrm{m}^{3}\right)$ and the thickness of all FPC engineering structure surrounding a plasma of radius a is $\Delta$; for maximum engineering power density $\Delta=r_{p}$. For example, $M P D=76.9 \mathrm{I}_{w} / \Delta$

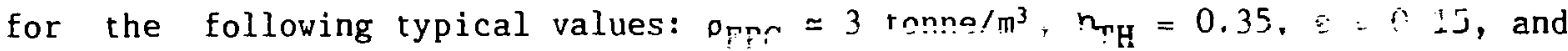
$M_{N}=1.3$. Taking as a goal UDC $\leq 1000 \$ / \mathrm{kWe}$ and the FPC contributing $\leq 10 \%$ to this cost, then MPD $\geq 10 \mathrm{c}_{\mathrm{FPC}}(\$ / \mathrm{kg})$. Typically (Table 1.1.- I), $\mathrm{c}_{\mathrm{FPC}} \geq 30 \mathrm{\$} / \mathrm{kg}$, which requires $M P D \geq 300 \mathrm{kHe} /$ tonne for this case; from Eq. (1.1.-3), $I_{W} \geq 5.9 \mathrm{MW} / \mathrm{m}^{2}$ for $\Delta \geq 1.5 \mathrm{~m}$, and $\mathrm{COE}=41 \mathrm{mills} / \mathrm{kWeh}$. This sample case is summarized in Table 1.1.-II. More detailed, but nonetheless approximate, generic models ${ }^{15}$ predict a minimum threshold of MPD $\geq 100-200 \mathrm{kWe} / t$ onne for competitiveness with fission power, beyond which little decrease in COE is expected from additional decreases in direct FPC costs; this prediction of an MPD threshold is substantiated by a number of specific fusion reactor designs, some of which are summarized in Table 1.1.-III, which includes the results from the present CSR study. As seen from Table 1.1.-I, a minimum decrease in the FPC mass of $\sim 2-3$ relative to the earlier fusion reactor designs will be required to achieve these improvements as measured in terms of reduced costs and sensitivity of these costs to the main physics requirements that in turn drive the FPC overall design. This behavior is shown graphically in Fig. 1.1.-2 using the results given on Tables 1.1.-I and 1.1.-III.

Increased MPD up to a point represents the most direct path for improvement in the cost position for fusion. For the geometrically optimal case [Eq. (1.1.-3), $r_{p} \simeq \Delta$, i.e., maximum first-wall area for minimum FPC volume], the MPD can be increased by increasing the plasma power density (i.e., $M P D \propto I_{w} / a \propto \beta^{2} B^{4}$ with the plasma power density being $P_{F} / V_{p} \simeq 1.2 \beta^{2} B^{4}$ ). Increasing MPD by this route requires increased efficiency of magnetic-field utilization (i.e., $\beta$ ), but increased magnetic field, $B$, will either increase the recirculating power (i.e., $\varepsilon$ ) if resistive coils are used or increase the magnet cost for either superconducting or resistive-coil FPCs, with both ultimately increasing $\mathrm{c}_{\mathrm{FPC}}(\mathrm{S} / \mathrm{kg})$. These tradeoffs, along with others to be mentioned, must be examined in the context of a specific confinement scheme and a selfconsistent reactor design. Designs that promote higher-power-density plasmas 
Net electric power, $P_{E}$ (MHe)

Plasma minor dimensions, $a / b(m)$

Plasma major toroidal radius, $\mathrm{R}_{\mathrm{T}}(\mathrm{m})$

Aspect ratio, $A=\mathrm{R}_{\mathrm{T}} / \mathrm{a}$

Plasma volume, $V_{p}\left(m^{3}\right)$

Sverage plasma density, $n\left(10^{20} / \mathrm{m}^{3}\right)$

Plasma temperature, T(keV)

PIasma energy, $w_{p}(G J)$

Field energy, $Y_{B}(G J)$

Total thermal power, $\mathrm{P}_{\mathrm{TH}}$ (MUt)

Recirculating pover fraction, $1 / 0_{E}$

Thermal conversion effictency, $n_{\mathrm{TH}}$

Net plant efficiency, $\eta_{p}=\eta_{T H}\left(1-1 / 0_{E}\right)$

Neutron flrst-vall loading, $I_{W}\left(H W / \mathrm{m}^{2}\right)$

Plasma power density, $\mathrm{P}_{\mathrm{F}} / \mathrm{V}_{\mathrm{p}}\left(\mathrm{HH} / \mathrm{m}^{3}\right)$

Average beta, $\theta$

Field at plasma, $B_{0}(t)(a)$

Field at coll, $B_{c}(T)$

Plasma thermal diffusivity, $\mathrm{x}_{E}\left(\mathrm{~m}^{2} / \mathrm{s}\right)$

Plasma current, $I_{\phi}(\mathrm{HA})$

Plasme current density, $j_{\phi}\left(M A / m^{2}\right)$

FPC volume, $V_{F P C}\left(m^{2}\right)$

FPC mass, $\mathrm{M}_{\mathrm{FPC}}$ (tonne)

FPC power density, $\mathrm{P}_{\mathrm{TH}} / \mathrm{N}_{\mathrm{FPC}}\left(\mathrm{HUt} / \mathrm{m}^{3}\right)$

Mass power density, $1000 \mathrm{P}_{\mathrm{E}} / \mathrm{H}_{\mathrm{FPC}}$ (kHe/tonne)

FPC unit $\cos t(\$ / \mathrm{kg})$

(FPC Cost)/TDC

Unti direct cost, UDC ( $\$ / k$ He $)^{(b)}$

Cost of electricity, $\operatorname{coE}(\mathrm{mill} / \mathrm{s} / \mathrm{kHeh})(b, c)$

\begin{tabular}{cc}
\multicolumn{2}{c}{ CRFPR } \\
\hline CRFPR(20) & CRFPR(5) \\
\hline 1000. & 1000. \\
0.71 & 1.42 \\
3.90 & 7.60 \\
5.5 & 5.5 \\
37.81 & 302.5 \\
6.55 & 2.3 \\
10. & 10. \\
0.12 & 0.34 \\
1.7 & -5. \\
$3,472$. & $3,609$. \\
0.20 & 0.22 \\
0.36 & 0.36 \\
0.29 & 0.28 \\
19.0 & 5.0 \\
70.4 & 9.6 \\
0.13 & 0.13 \\
5.2 & 3.0 \\
$3.0-4.0$ & $2.5-3.0$ \\
0.41 & 0.54 \\
18.4 & 21.6 \\
11.6 & 3.4 \\
359. & $1,042$. \\
$1,117$. & $-2,000$. \\
9.7 & 3.5 \\
895. & -500. \\
45. & 42. \\
0.042 & 0.05 \\
$1,111$. & $31.0 / 55.5$ \\
$27.9 / 49.9$ & \\
& \\
10.4 \\
0.4
\end{tabular}

\begin{tabular}{|c|c|}
\hline \multicolumn{2}{|c|}{ ATR/ST } \\
\hline ATR/ST & ATR/ST \\
\hline 500. & 1000 \\
\hline $1.50 / 4.50$ & $1.50 / 4.50$ \\
\hline 2.70 & 2.70 \\
\hline 1.8 & 1.8 \\
\hline 358. & 358. \\
\hline 1.21 & 1.63 \\
\hline 15. & 15. \\
\hline 0.32 & 0.43 \\
\hline 12. & 16. \\
\hline 2,047 & 3,710 \\
\hline 0.32 & 0.25 \\
\hline 0.36 & 0.36 \\
\hline 0.24 & 0.27 \\
\hline 3.24 & 5.87 \\
\hline 4.7 & 8.5 \\
\hline 0.291 & 0.291 \\
\hline $4.10(2.30)$ & $4.77(2.67)$ \\
\hline 6.9 & 8.0 \\
\hline 0.53 & 0.72 \\
\hline 39.8 & 46.2 \\
\hline 1.9 & 2.2 \\
\hline 2,102 & 2,120 \\
\hline 6,344 & 6,492 . \\
\hline 1.0 & 1.8 \\
\hline 79. & 154. \\
\hline 45. & 45. \\
\hline 0.24 & 0.20 \\
\hline 2,441 & 3.485 \\
\hline $61.4 / 116.0$ & $37.8 / 71.1$ \\
\hline
\end{tabular}

\begin{tabular}{|c|c|}
\hline \multicolumn{2}{|c|}{$\operatorname{csR}$} \\
\hline 500. & 1000 \\
\hline $0.83 / 2.77$ & $1.12 / 3.72$ \\
\hline 1.39 & 1.89 \\
\hline 42.9 & 105.2 \\
\hline 2.5 & 2.3 \\
\hline 20. & 20. \\
\hline 0.10 & 0.23 \\
\hline 0.7 & 1.5 \\
\hline 1,803 & 3,416 \\
\hline 0.21 & 0.16 \\
\hline 0.36 & 0.36 \\
\hline 0.28 & 0.30 \\
\hline 18.7 & 19.8 \\
\hline 33.6 & 26.0 \\
\hline 0.10 & 0.10 \\
\hline 5.4 & 5.0 \\
\hline 2.8 & 2.6 \\
\hline 0.45 & 0.73 \\
\hline 37.5 & 47.3 \\
\hline 6.1 & 4.3 \\
\hline 177. & 321. \\
\hline 521. & 820. \\
\hline 10.2 & 10.7 \\
\hline 960. & 1,200 \\
\hline $\begin{array}{l}53 . \\
0.039\end{array}$ & $\begin{array}{l}53 . \\
0.047\end{array}$ \\
\hline $\begin{array}{l}1,481 . \\
37.5 / 67.0\end{array}$ & $\begin{array}{l}978 . \\
25.0 / 44.7\end{array}$ \\
\hline
\end{tabular}

(a) Values in parentheses are on-axis vacuum fields, values for CRFPR and CSR correspond to plasma edge, outboard equatorial plane.

(b) 19 go dollars

(c) Constant/then-current dollars based on a 6-year construction time and a nominal $76 \%$ plant avallability, which

la actualiy determined from neutron wall loading and assumed integrated radiation $11 \mathrm{fe}\left(20 \mathrm{MWyr} / \mathrm{m}^{2}\right)$. 


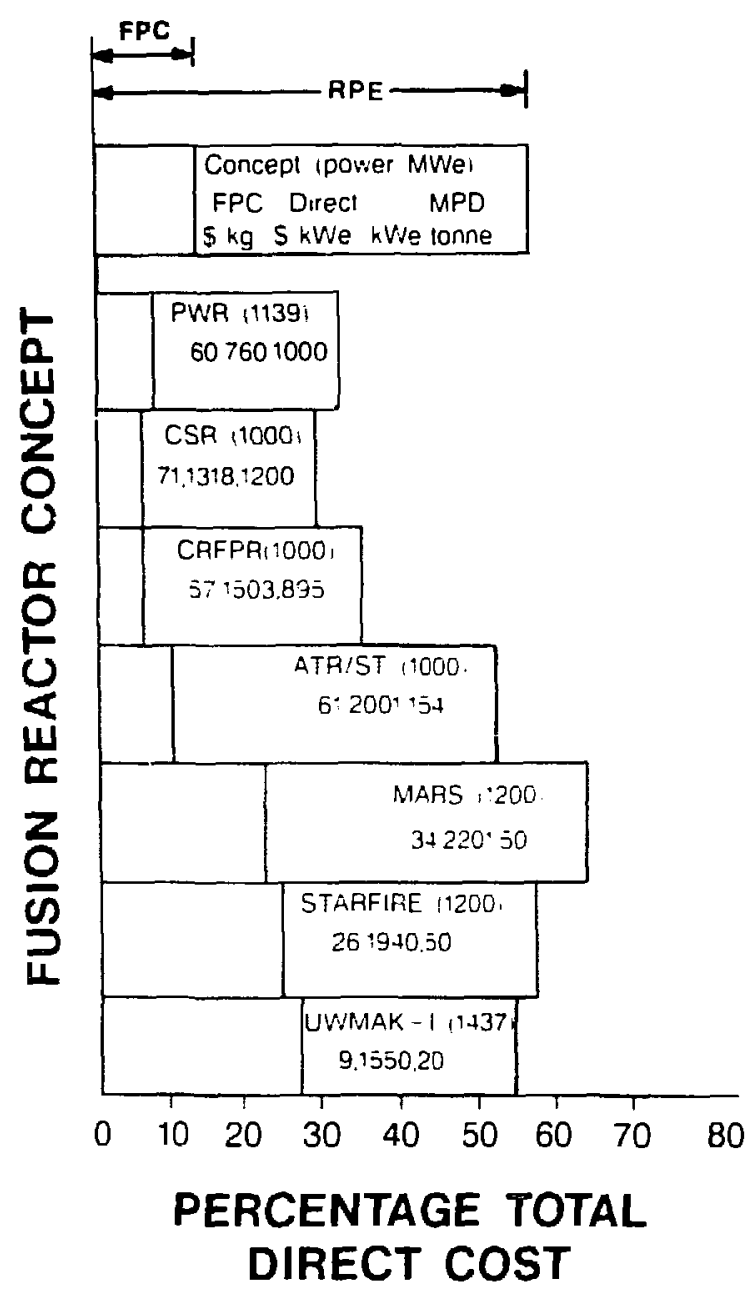

Fig. 1.1.-2. Percentage of total direct cost devoted to the fusion power core (FPC) and Reactor Plant Equipment (RPE, Account 22.) for the range of older and more recent fusion power plant designs summarized on Tables 1.1.-I and 1.1.-III. The results of the present CSR study are also showr.

while limiting the total power will require better plasma confinement efficiency, $X_{E}$, in plasmas of smaller dimensions (total fusion power, $P_{F} \propto X_{E}^{2} R_{T} / r_{p}$ for $n \tau_{E} T \propto 6 B^{2} r_{p} / X_{E}$ nominally constant). In addition to placing more demands on physics through increased $\beta$ and decreased $X_{E}$, the achievement of direct cost reductions and insensitivity to FPC physics and technology through increased MPD can also have impact on costs in areas other than those noted above. These impacts are summarized as follows: 
- Increased $I_{W}$ leads to increased nuclear-afterheat power density, decreasing the degree of assured safety and possibly adding costs associated with added plant safety systems.

- Increased $I_{w}$ may be accoinpanied by increased heat flux, perhaps requiring special high-heat-flux materials, adding to FPC unit cost and possibly limiting materials choices, particularly as related to the desire to reduce long-term radioactivity.

- Increased $I_{w}$ may require separate surface (first wall, limiter) and bulk-heating (blanket) coolants, decreasing $\eta_{\mathrm{TH}}$ and adding to FPC, RPE, and BOP unit costs.

- Already noted is the possibility for increased recirculating power fraction, $\varepsilon$, if thin blankets and/or resistive coils are utilized to increase MPD; the tradeoff associated with FPC versus $\varepsilon$ is strongly dependent upon concept. Increased $\varepsilon$ will also lead to increased BOP thermal ratings and associated costs.

On the other side of the ledger, however, smaller higher-power-density :FPCs offer the potential for a number of improvements beyond the reduction of direct cost. 位provements envisaged for MPD beyond the COE-based threshold are listed as follows:

- Increasea FPC operational flexibility

- single-(or few-) piece maintenance of the reactor torus

- ability to sustain and recover from significant FPC breakdowns

- ability to conduct significant testing on a fully assembled FPC prior to nuclear service to increase operational reliability

- ability to incorporate innovation and improve FPC throughout plant life 
- Reduced impact of physics and technology uncertainties on overall cost of fusion power

- More rapid development of "learning curves," more closely coupled feedback tr, developing experience base, early assembly of reliability database

These advantages, although not directly reflected in present models used to compute COE, nevertheless combine to promise a generally less expensive, bolder, and faster development path towards a competitive fusion end-product.

1.1.2. Utility-Based Issues for Improved Fusion Reactors. The main emphasis in assessing the prospects for and means to improve fusion has been placed on quantitative cost estimates as well as less-quantitative assessments of complexity as related to plant availability and overall operational risk. In addition to capital and life-cycle energy cost, however, the attractiveness of a new energy source depends strongly on construction lead-times and financial risks related both to protracted construction and licensing periods and to capital-cost overruns. Although more difficult to quantify, the impact of these important but highly variable (in space and time) forces can be important.

A present-day U.S. : utility contemplating a new energy source will generally try to minimize risks to itself by transferring these risks to the constrgers in the form of high fuel charges or to the reactor vendor in the form of "turn-key" operation. The optimal size of a power plant from the utility perspective depends largely on the utility struc:.re (and, hence, locale), with incremental supplies totaling not more than $10 \%$ of the total grid being desirable. The appeal of the fusion reactor, like that of fission today, 30 is expected to be sensitive to properties like the optimal unit size or capacity, predictability of direct costs, construction lead-time, plant reliability, and risks of long-Lerm outages. If a new plant were available to the utility at low overall cost, substitution of new capacity for aged and uneconomic units would be encouraged, and this new capacity would create forces to decrease the cost of energy, increasing both demand and the capacity to fulfill it.30

On the other hand, if large capital outlays combine with long lead-time, as is the case presently in the U.S., the utility will minimize financial risks by constructing short-lead-time, low-capacity ( $\leq 300-\mathrm{MWe}$ ) plants, or more likely emphasize conservation, better load-management, extension of existing (aged) 
plant life, and use of short-term, high-fuel-cost generation options; in the U.S., at least, these fuel costs are passed through to the consumer and immediately impact the rate base, unlike the time-related costs of delayed construction.

It is informative to interpret the recent misfortunes of the nuclear power industry in the U.S. in terms of the fusion goal. These problems have recently been summarized 31 and can be related to a lack of radwaste disposal, performance that is below expectations, and, most importantly, increases in capital costs that far offset lower fuel costs. Fusion may very well have to deal with similar problems of high capital cost, and the causes for and means to solve these problems are worthy of note. Increased capital cost of fission power in the $19 \mathrm{Tn}_{\mathrm{S}}$ resulted from: a) increased construction lead-time in a period of high inflation and interest rates, and b) direct capital costs that increased at an annual rate of $\sim 8 \% .{ }^{31}$ Both a decrease in productivity and the unique technological/organizational/managerial/regulatory reìtionships for the nuclear industry in the U.S. contributed to this increased cost. The concern over safety of nuclear plants results in more subsystems and components, increased design and component reviews, extensive documentation and records, and more retrofitting of completed systems. This situation coupled with the somewhat disjointed U.S. nuclear structure (i.e., utility, reactor vendor, architectengineering, primary contractor, regulatory agencies) creates a situation where the regulatory agency rather than the user leads the process, innovation and improvement (i.e., change) are discouraged, technology never bicomes standardized, and crucial experience is not allowed either to build up or to be transferred. A number of possible cures for these problems have been suggested:31 a) reduce the capacity of each unit to counter "diseconomies of scale" related both to extended lead-time and pcor plant performance of large nuclear power plants; b) achieve better quality control through increased factory fabrication and decreased plant-site construction; c) adopt passive, inherent, or "walk-away" safety philosophies to reduce the need to demonstiate the effectiveness of active safety systems; or d) dismantle institutional barriers while continuing to build on an extensive construction and operational experience with LWRs; e) buy foreign, qualified systems.

These utility-based and institutional issues are expected to shape strongly, but less-quantitatively, the direction for improved fusion systems. The present fashion in the U.S. towards small, short-lead-time power systems 
may be short-termed relative to the time scale for the commerrialization of fusion, but nevertheless these trends shape a window for fusion that may be difficult to meet by present concepts as more advanced, economic fission systems are proposed. Although the long-range nature of fusion power makes reasonable the focus on improvements in UDC and COE, rather than the issues of small capacity and utility acceptance based on present-day financial pressures and energy demands, nevertheless, the role of fusion eventually will be more strongly shaped by and must be cognizant of these utility-related issues, particularly as they relate to capacity, complexity, reliability, and licensibility.

\subsection{Approaches to Improved Fusion Reactors}

Figure 1.2.-1 depicts the main classes of magnetic confinement systems presently under worldwide study. This diagram emphasizes approximate relationships between concepts, with systems supporting large plasma currents positioned on the left and those containing little or no plasma current being positioned on the right. The latter systems, including the present vision of the tokamak and the tandem mirror, are dominated by externally imposed axial or toroidal magnetic fields and, therefore, considerations of overall plant efficiency generally lead to the use of large superconducting coils. Confinement systems located on the left side of Fig. 1.2.-1 support more of the plasma pressure by iriternal plasma currents, are to varying degrees poloidalfield dominated (PFD), have reduced requirements for externally imposed magnetic fields, and to varying degrees can operate with efficient resistive (copper or aluminum alloy) coils; these PFD concepts require minimal blanket and shield thicknesses compared to superconducting systems, and a considerable reduction in the FPC mass, size, and complexity is envisaged. The possible disadvantages of the PFD systems are the need to sustain plasma currents, the need for a conducting shell near the generally high-beta plasma, and a physics database that is not as well developed as for the "conventional" tokamak.

To varying degrees, the advanced tokamaks (i.e., ST and ET in Fig. 1.2.-1) exhibit PFD-like characteristics, with the efficient use of resistive copper coils to confine higher-beta, higher-power-density plasmas also promising reductions in FPC size, mass, complexity, and cost. Although the tokamak physics database far exceeds that for many of the other approaches listed on Fig. 1.2.-2, the advanced tokamak embodiments (including SSR variants) must 


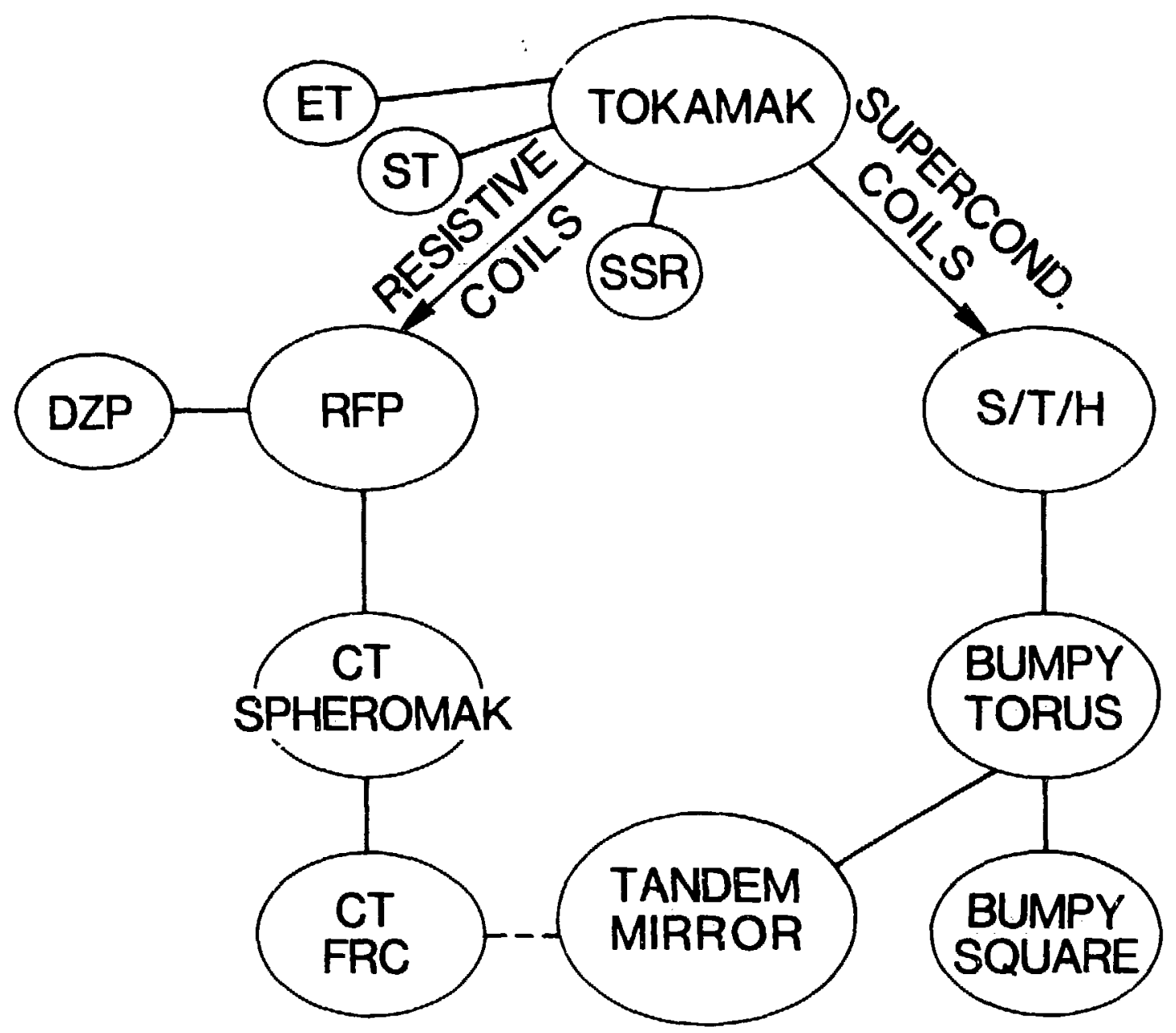

Fig. 1.2.-1. Options for magnetic fusion. The higher-beta options for the tokamak include the spherical torus, $\mathrm{ST}^{24,32}$; the elongated torus, $\mathrm{ET}^{33}$; and operation in the second stability region, SSR. ${ }^{4}$ The stellarator, torsatron, and heliotron systems are grouped as $\mathrm{S} / \mathrm{T} / \mathrm{H} .7,8,35,36$ As for the $\mathrm{S} / \mathrm{T} / \mathrm{H}$, the bumpy torus ${ }^{9}$ can be viewed in terms of plasma confinement on drift surfaces, this usually large system projecting compactness when formed into a sauare or high-order polyhedron. ${ }^{37}$ The reversed-field pinch, RFP $2,26,41,42$ is the first significant step away from the "conventional" tokamak as a PFD system. The Dense Z-Pinch, DZP3 8 and compact toroid (CT) spheromak $^{27}$ have no toroidal or axial field outside the plasma. The field-reversed configuration, FRC, ${ }^{39}$ is a CT with no toroidal field, either inside or outside the plasma. The tandem mirrorlo,40 embodies characteristics of both FRCs, S/T/Hsd, and bumpy tori/squares, including the use of high-field superconducting and resistive coils, drift surfaces, energetic electron rings, and linear central geometry. 

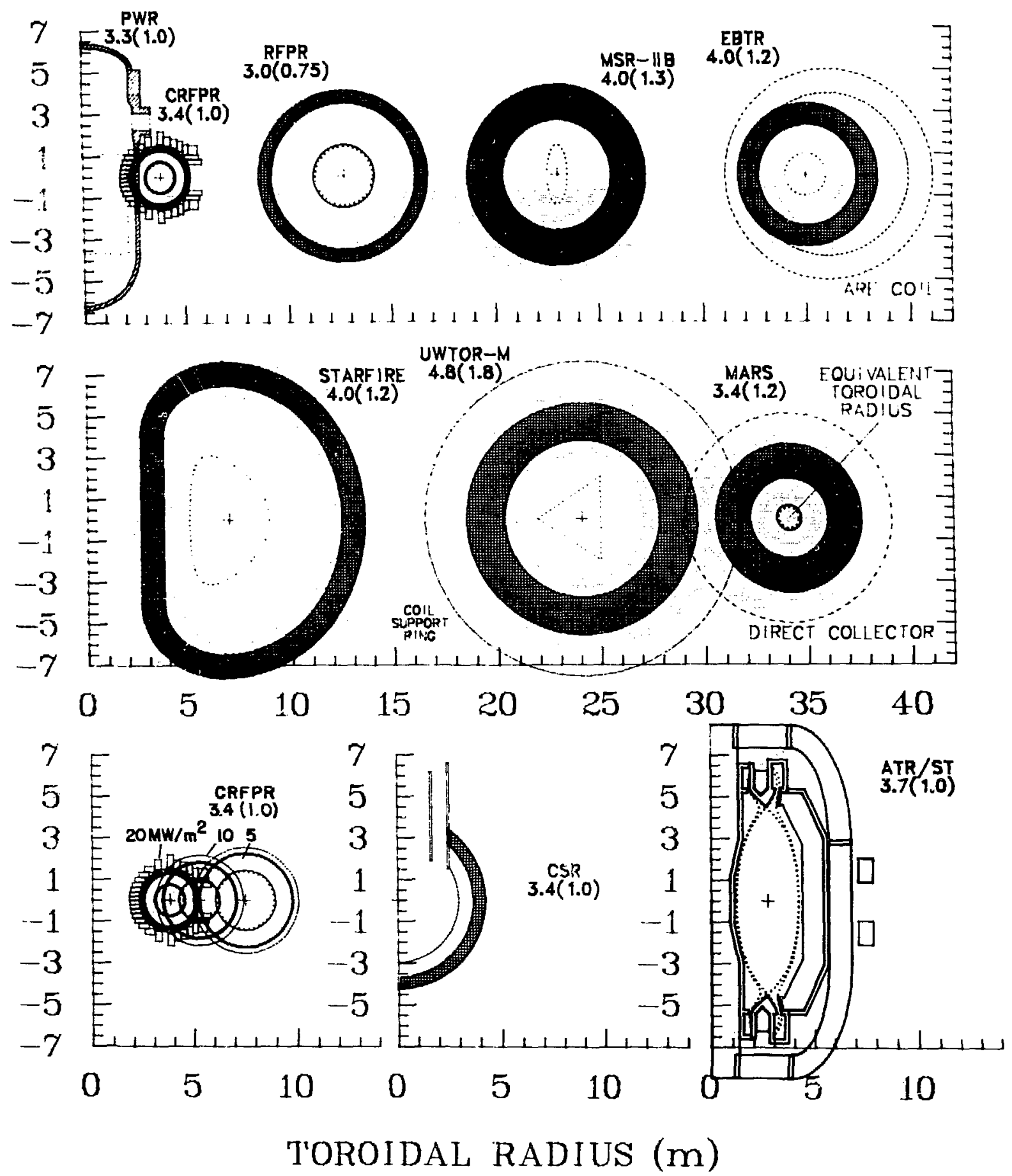

Fig. 1.2.-2. Cross-section of FPCs For a range of fusion reactors, including the RFP, spheromak, and ST concepts summarized on Table 1.1.-III. 
extrapolate significantly from present understanding into regions where other concepts have equal if not stronger databases. This situation couples with: a) the transferability of physics understanding across concept boundaries; b) strong experimental successes, particularly for RFPs, spheromak CTs, and FRC CTs; and c) the possibility to extend these latter concepts to viable commercial end-products without ever-increasing plasma sizes to project significant improvements in systems located on the PFD (left) side of Fig. 1.2.-1. For these reasons, the prognoses for improved reactors have focused most recently on systems like the RFP, spheromak, and the ST tokamak. Fig. 1.2.-2 gives crosssections of the RFP, spheromak, and ATR/ST reactors, along with comparisons to past reactor designs (Tables 1.1.-I and 1.1.-III). The view of the compact spheromak reactor (CSR) given in Fig. 1.2.-2, is a result of the preliminary scoping study reported herein.

\subsection{Overview of Spheromak Concept}

A CT is an axisymmetric torus that has no magnet coils, conducting walls, or vacuum surfaces linking the torus. With only poloidal field and in an elongated (prolate) form required for stability, the high-beta (0.8-1.0) FRC results. The spheromak is a CT with both poloidal, $\mathrm{B}_{\theta}$, and toroidal, $\mathrm{B}_{\phi}$, fields, and, like the RFP, both field components in the oblate spheromak are comparable in magnitude and are generally configured into a near-minimum-energy state.43,44 Spheromaks have been generated using magnetized co-axial plasma guns $\left[C T X, 45\right.$ BETA-II $\left.{ }^{46}\right]$, combined fast-pulsed $Z$ - and $\Theta$-pinch techniques (PS-1), 7 and Electrodeless flux-core formation techniques (S-1).48 Reactor projections have been made for spheromaks formed by flux-core, 49 with formation by magnetized$\operatorname{gun}^{27}$ techniques being a focus of the present study.

In addition to the attributes of strong ohmic heating, high plasma and even higher engineering beta (i.e., plasma pressure relative to magnetic-field pressure at the confining coils), and the efficient use of resistive (equilibrium) coils to give a high MPD, the simply connected CT magnetic geometry (i.e., no conductors passing through the torus) further reduces the impact of the FPC on the overall cost equation for fusion. Formation techniques based on a magnetized co-axial electrode also promise an exo-reactor divertor lor impurity control as well as the proper arrangement of electrodes to inject linked magnetic fluxes (i.e., magnetic helicity) with an externally applied dc voltage; dc current drive through divertor-plate-like electrodes immersed only 
in the plasma scrapeoff may be possible. Hence, toroidal flux emerging from the magnetized-gun electrodes links a small fraction of poloidal flux at the outer flux surfaces and magnetic helicity is injected at a rate required to sustain the plasma against resistive decay of the magnetir ronfiguration as well as supplying power losses incurred in the divertor and the edge-plasma regions. Experimental evidence has been reported for such sustainment over ten magneticenergy decay times. ${ }^{45}$ Generally, picsent-day spheromaks sustained by electrodes have a higher impurit $y$ content and poorer confinement than spheromaks operated in the detached or separated mode. The development of cleaner and more energy efficient electrode systems represents key areas of research. Generally, the spheromak represents a logical and attractive extension from the already promising reactor improvement projected for the RFP and represents the focus of this study. The CSR parameters listed in Tables-1.1.-I and 1.1.-IIT, as well as the geometry illustrated in Fig. 1.1.-2, correspond to the minimum-COE, $P_{E}=1,000-M W e(n e t)$ results from the study report sd herein. 


\section{STUDY BACKGROUND AND APPROACH}

\subsection{Background}

Encouraging experimental results from the smaller, less capital-intensive fusion confinement schemes are summarized in Table 2.1.-I, which also gives comparisons with key stellarator and tokamak devices. Figure 2.1.-1 illustrates this progress on the Lawson diagram. The relationship between these experiments is illustrated graphically in Fig. 2.1.-2, which shows the toroidal fusion concepts arranged according to the magnitude of plasma self-current, $I_{\phi^{*}}$. Specific profile information is qualitatively compared in Fig. 2.1.-3, which depicts toroidal and poloidal fields, $B_{\phi}$ and $B_{\theta}$, respectively, along with characteristic aspect ratios, $A \equiv \mathrm{R}_{\mathrm{T}} / \mathrm{r}_{\mathrm{p}}$, of each concept. Stellarators operate

TABLE 2.1.-I

RESULTS FROM THE KEY TOROIDAL FUSION EXPERIMENTS(a)

\begin{tabular}{|c|c|c|}
\hline $\begin{array}{c}\beta \\
\text { Peak (ave) } \\
\end{array}$ & $\begin{array}{c}n \tau_{E} \\
\left(10^{16} \mathrm{~s} / \mathrm{m}^{3}\right) \\
\end{array}$ & $\begin{array}{l}\mathrm{T}_{\mathrm{i}} \\
(\mathrm{keV})\end{array}$ \\
\hline 0.01 & $240^{(b)}$ & 1.0 \\
\hline 0.01 & 250 & 1.0 \\
\hline
\end{tabular}

TOKAMAK

Alcator C (MIT) $)^{52}$

$\begin{array}{llcc}0.015 & 8,000 & 1.5 & 0.8 \\ 0.064(0.02) & 400 & 5.0 & 0.9 \\ 0.005^{5} 5 & 1,900 & 10 . & 2.5 \\ \leq 0.005^{55} & 1,250 & 8 . & 4.8\end{array}$

Doublet III $(\mathrm{GA})^{53}$

TFTR (PPPL) 54

JET $(\text { EEC })^{54}$

0.25

10

0.5

0.5

ZT-40 (LANL) ${ }^{57}$

0.17

6

0.3

0.34

SPHEROMAK

CTX (LANL) 58

0.14

0.4

0.30

1.1

S-1 (PPPL) $)^{59}$

FIELD-REVERSED CONFIGURATION
FRX-C (LANL) 60
0.92
40
0.12
1.3
TRX-2 (MSNW) $)^{61}$
0.92
27
0.45
0.8

\footnotetext{
(a) The results presented in this table reflect conditions primarily in 1984; progress in the near term for certain experiments, particularly for the TFTR and JET devices (re: Fig. 2.1.-1), is also given.

(b) Excludes radiation
} 


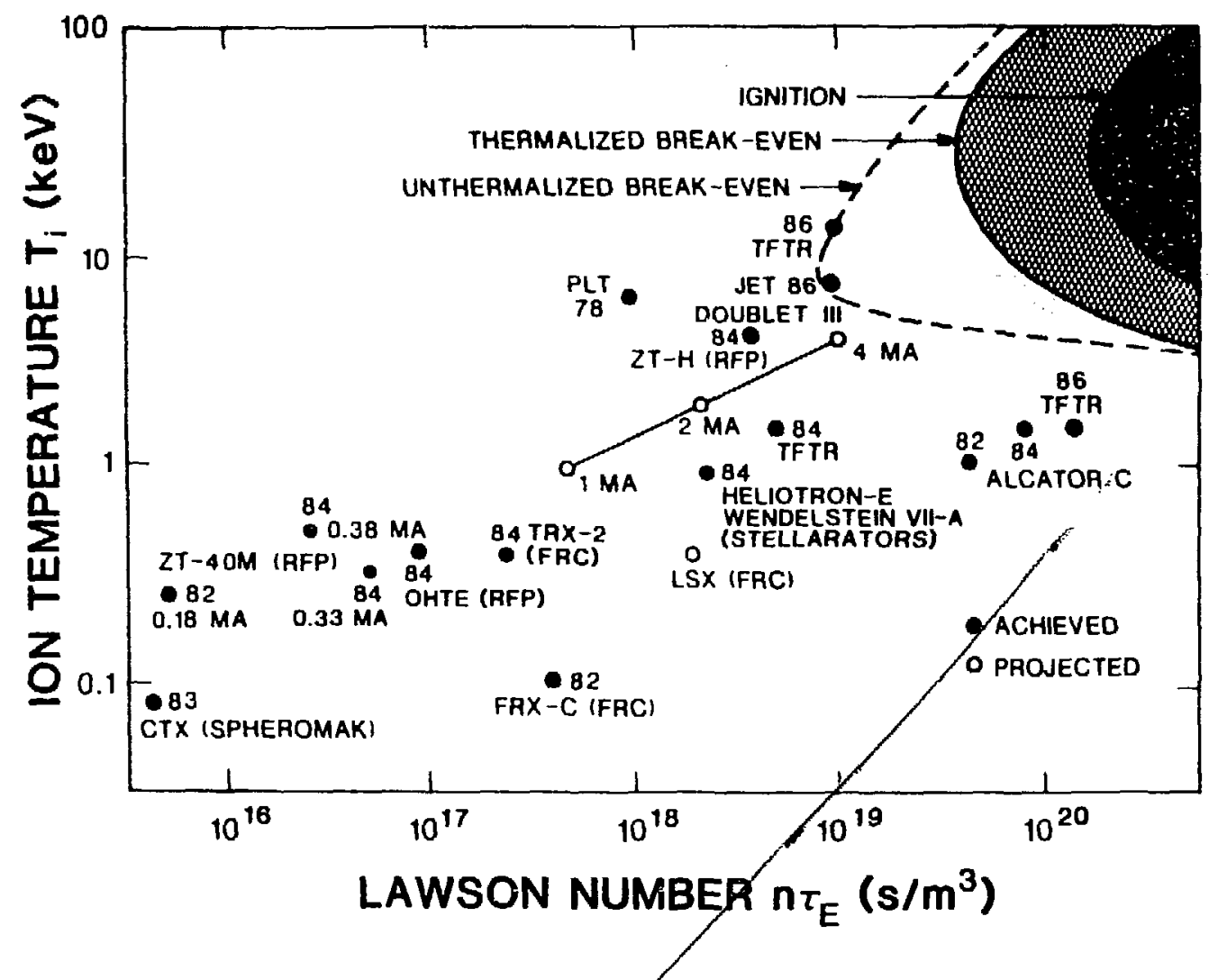

Fig. 2.1.-1. Lawson plot of confinement parameter $n \tau_{E}$, versus ion temperature for recent experiments. Shown also are recent (1986) TFTR results as well as projected performance for FRC(LSX) and RFP(ZT-H) devices.

with strong toroidal magnet cgrl fields, $B_{\phi}$, and no net toroidal current, $I_{\phi}$, while tokamaks have similar/strong magnet fields and a nominal toroidal current. Both concepts have difficulty operating with resistive magnet coils because of a) the high magnet felds and currents external to the plasma and b) typically low plasma betas. Fhen projected to a reactor using resistive coils, the power consumption in the coils is large, and superconducting coils are invoked to avoid the correspondingly high-recirculating power. Thick structures $(\sim 2 \mathrm{~m})$ are generally required to shield these superconducting coils from thermal flux and radiation damage. Accommodating this structure on the inner portion of the reactor torus inflates the size of the entire system, leading to FPCs of 25-45 ktonnes for a - 1000-MWe system ${ }^{-10}$; these masses of the primary heat squice are similar to a naval missile cruiser (25 ktonnes) or battleship (40 ktonnes), 62 and compare to $\sim 1$ ktonne for the comparable system in a PWR.63 


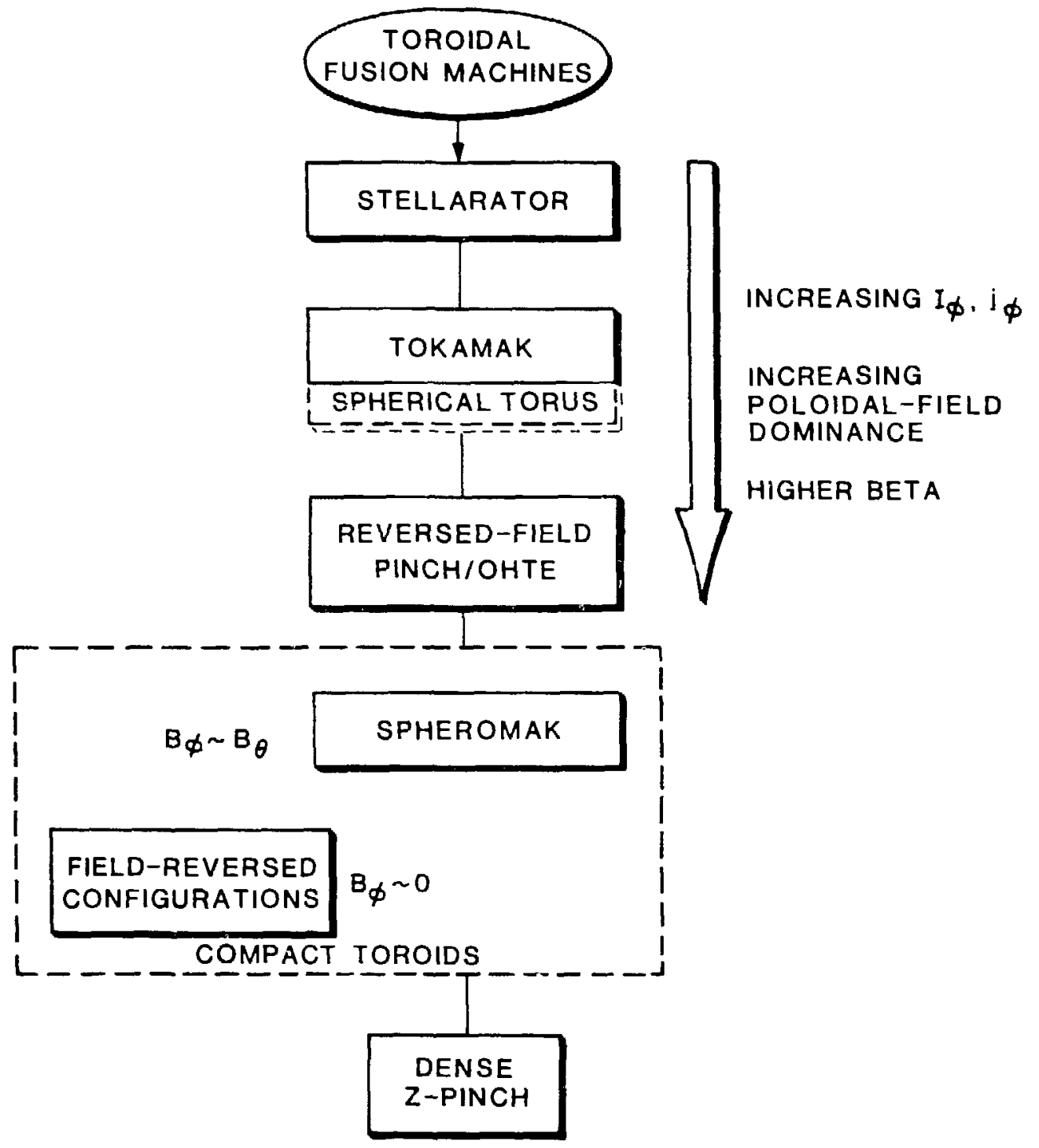

Fig. 2.1.-2. Family of toroidal plasma confinement concepts arranged in order of increasing plasma self current.

Since an entire 1000-MWe nuclear fission plant, including all steel reinforcing bar but not concrete per se, weighs approximately $40 \mathrm{ktonnes,63}$ the addition of a higher-technology (when compared to the balance of plant) fusion unit of the 25-40 ktonne class is expected to increase the cost of the electric power producing unit by at least a factor of 1.5-3.0 when compared to fission for "tenth-of-a-kind" systems; even greater factors are expected for 

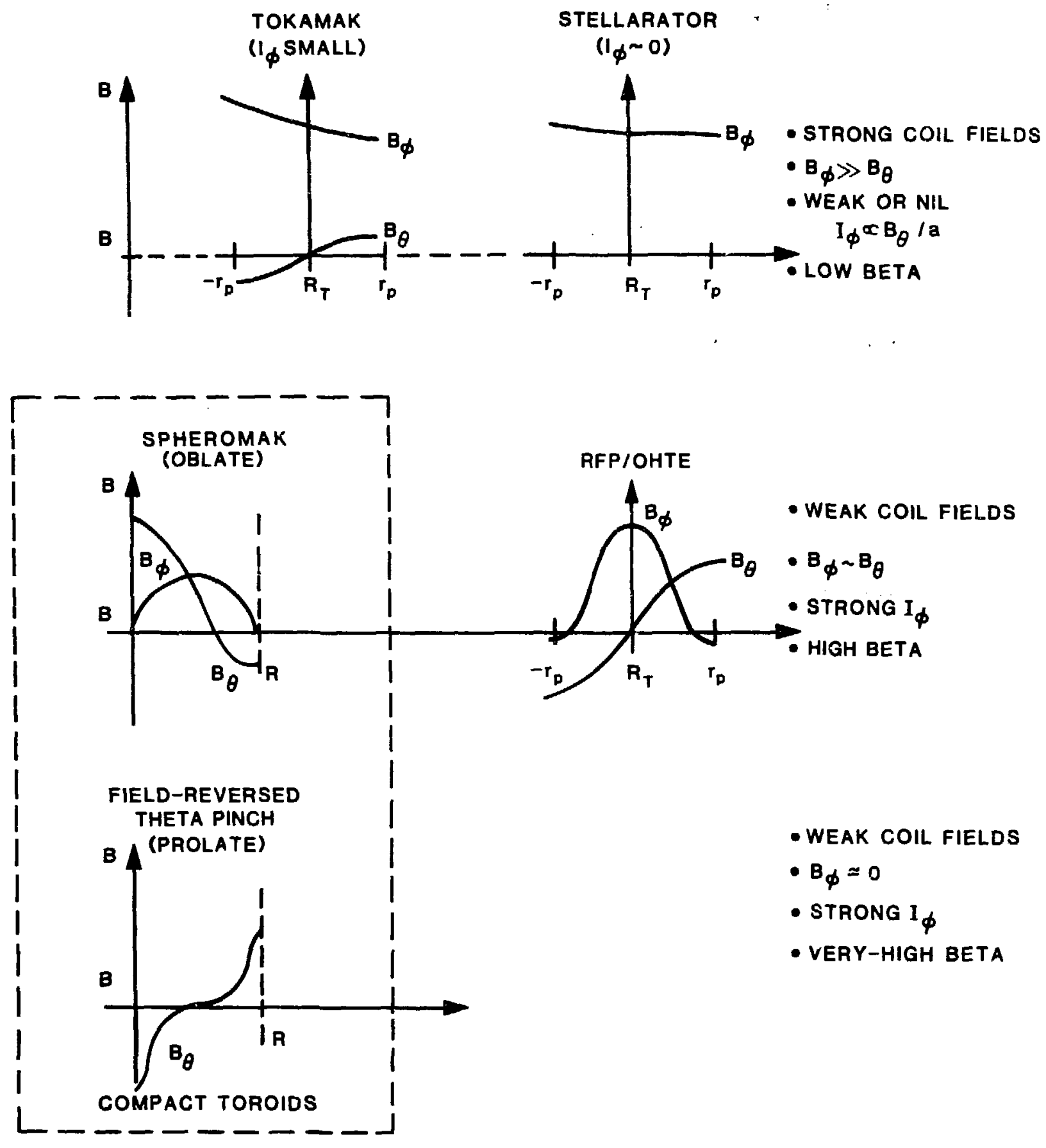

Fig. 2.1.-3. Family of toroidal fusion concepts showing toroidal, $B_{\text {, }}$ and poloidal fields, ${ }_{\theta}$, along with the relative aspect ratios, $\mathbb{R}_{\mathrm{T}} / \mathrm{r}_{\mathrm{p}}$. 
"first-of-a-kind" units, bringing into question whether fusion power can be afforded in spite of attractive fuel and safety/environment attractions.

Plasma confinement-concepts that rely on large plasma self-currents, such as RFPs, OHTEs, and spheromaks, operate with appreciably reduced toroidal magnetic fields, with the spheromak having no exo-plasma toroidal field, as is illustrated ir Figs. 2.1.-3 and 2.1.-4. Such systems can then use resistive magnets without serious penalty, thereby allowing designs with much thinner neutron-moderating blankets. This option allows the entire FPC and related systems to shrink in size, leading to FPC masses of $1-2 \mathrm{ktonnes}$ and

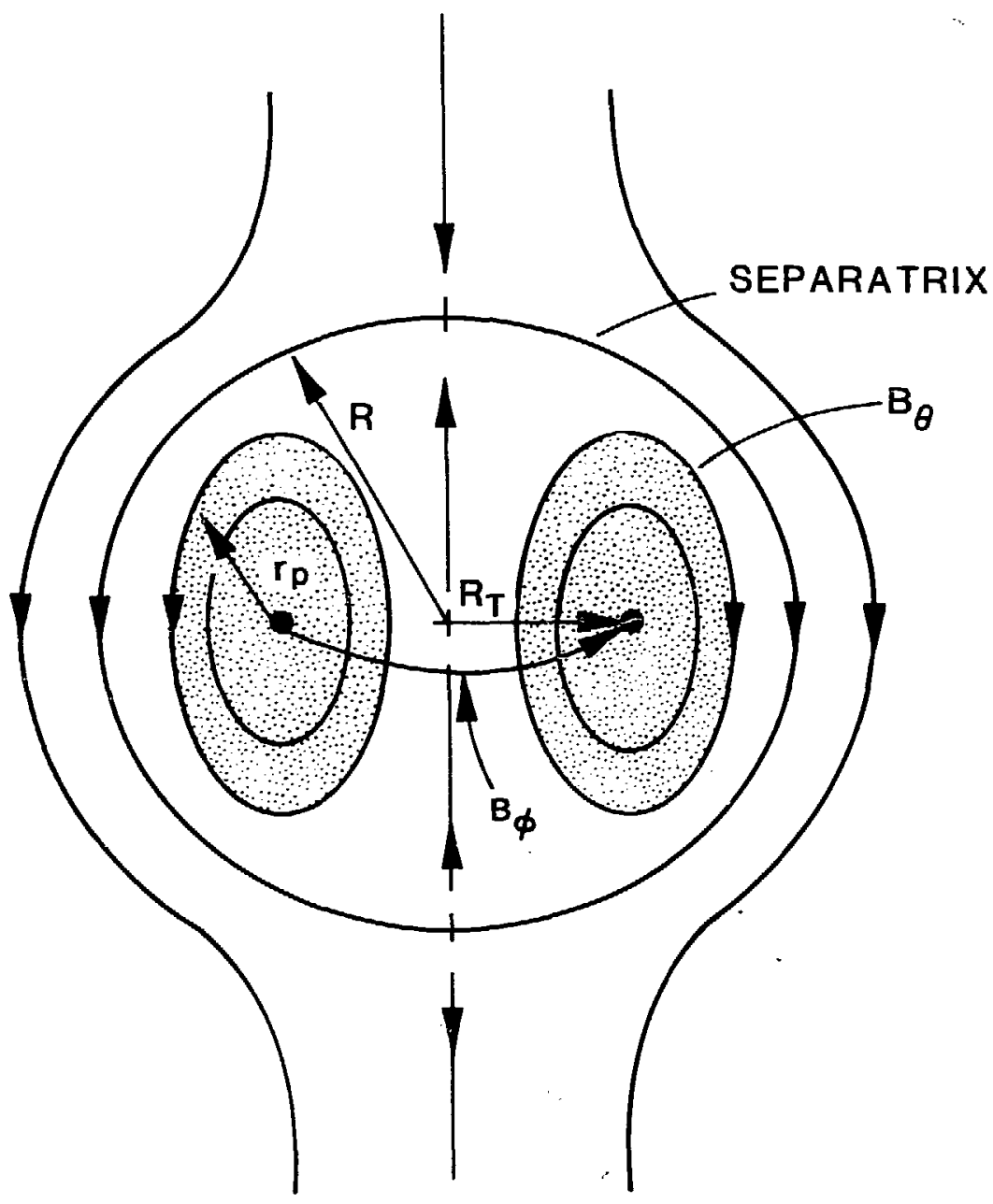

Fig. 2.1.-4. Spheromak plasma configuration showing the major toroidal radius, $\mathrm{R}_{\mathrm{T}}$, minor radius, $\mathrm{r}_{\mathrm{p}}$, and separatrix radius, $\mathrm{R}$, along with the toroidal, $\mathrm{B}_{\phi}$, and pololdal field, $\mathrm{B}_{\theta}$. 
correspondingly reduced system costs. Additionally, the benefits of few- or single-piece maintenance of the FPC become accessible, with the possibility of achieving the value of $\mathrm{p}_{f}$ assumed by most conceptual reactor designs. These generally positive conclusions reached for the compact RFP reactor system ${ }^{25} \operatorname{are}$ investigated for the spheromak configuration.

The specific spheromak configuration studied as a compact reactor has been selected from the options listed in Table 2.i.-II. Since the spheromak plasma is not interlocked by coil structures, translation of the plasmoid is possible.

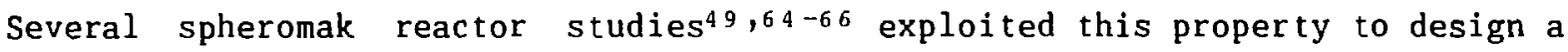
reactor that translated the plasma through a plasma chamber much longer $(>10$ times) than the plasmoid height. Several disadvantages can be identified for this plasma configuration, which, as is illustrated in Fig. 2.1.-5, must be oblate ${ }^{67}$ to satisfy equilibrium/stability constraint. The difficulty in maintaining the required shape in a translating burn chamber raises serious questions for this approach. In addition, translating-plasmoid designs necessarily lead to large systems because of the long FPC length compared to the plasma height. Finally, such designs necessarily must operate with a pulsed first wall and plasma because of the difficult task of refluxing these moving plasmoids. In this regard, the possibility exists for merging plasmoids, although plasma losses during such large-scale magnetic reconnections present a concern.

TABLE 2.1.-II

RATIONALE FOR CHOICE OF SPHEROMAK CONFIGURATION BASED ON THE GOAL OF STEADY-STATE COMPACT REACTOR CONFIGURATION CONSISTENT WITH PLASMA PHYSICS REQUIREMENTS

\begin{tabular}{|c|c|c|c|c|}
\hline APPROACH/ISSUE & STABILITY & EQUILIBRIUM & STEADY-STATE & COMPACT \\
\hline $\begin{array}{l}\text { Translating plasmoid; } \\
\text { PG\&E/LLNL,64, PPPL, } 49 \\
\text { KARIN-1,65 UI66 }\end{array}$ & $?$ & No & $?$ & Unlikely \\
\hline $\begin{array}{l}\text { Stationary plasmoid } \\
\text { inductively produced } \\
\text { and translated; } \text { PPL }^{49}\end{array}$ & $?$ & Yes & $\begin{array}{l}\text { Merged } \\
\text { Plasmas }\end{array}$ & $?$ \\
\hline $\begin{array}{l}\text { Stationary plasmoid gu } \\
\text { produced/driven LANL, } \\
\text { Japan }^{65}\end{array}$ & $?$ & yes & Yes & Probably \\
\hline
\end{tabular}




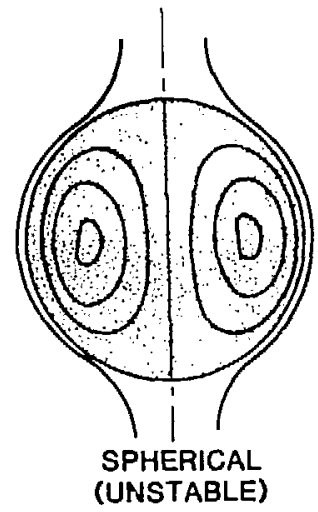

(UNSTABLE)
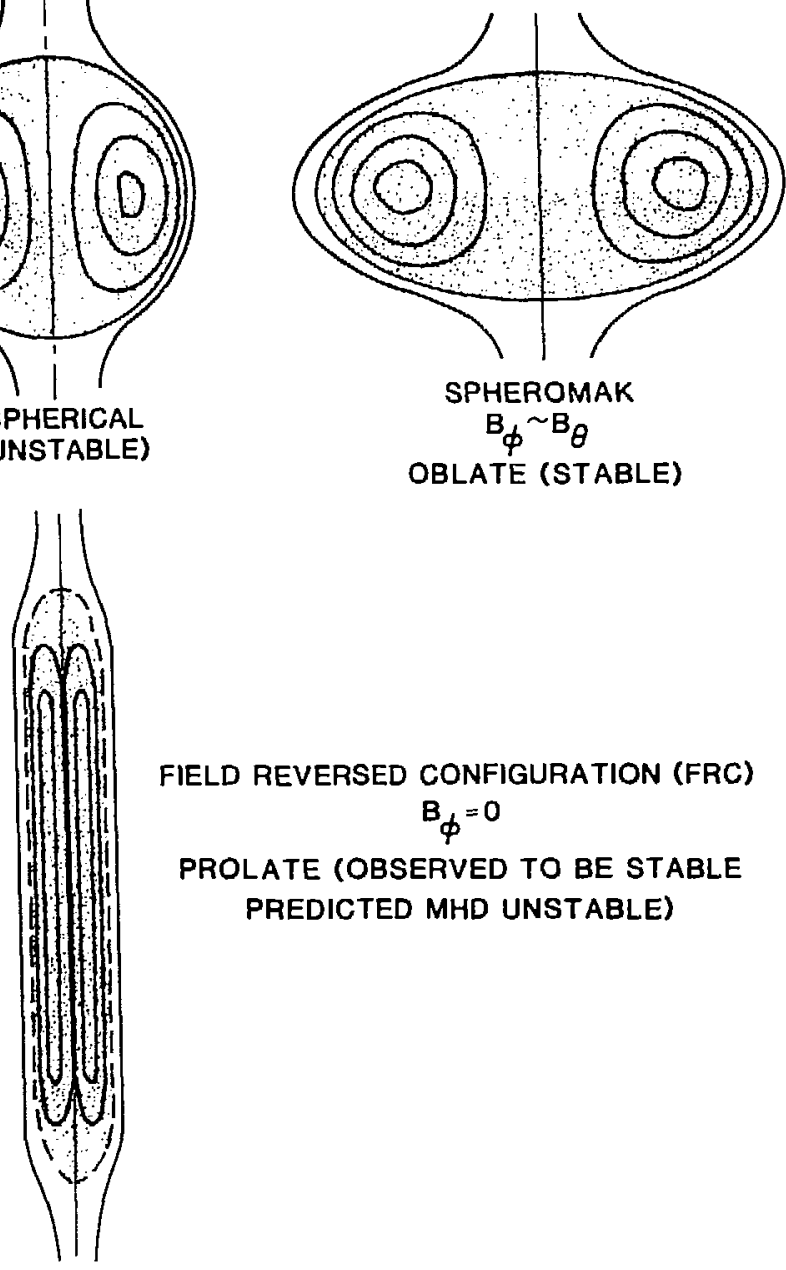

FIELD REVERSED CONFIGURATION (FRC)

$B_{\phi}=0$

PROLATE (OBSERVED TO BE STABLE

PREDICTED MHD UNSTABLE)

Fig. 2.1.-5. Various types of compact toroids with an oblate (stable) spheromak shown.

The spheromak equilibrium is maintained by constraining the stationary plasma inside a relatively close-fitting conducting shell or liner. Such stationary reactor systems have been proposed using both inductively produced 99 and gun-produced ${ }^{6,69}$ spheromaks. Stationary-plasma spheromak reactor designs may resolve the question of equilibrium, although steady-state can be achieved only if the magnetic configuration can be refluxed. Inductively produced plasmoids may be merged, as previously suggested for the translating-plasmoid approaches, although the trauma caused by breaking and reconnecting field lines 
may seriously increase losses. The refluxing issue may be resolvable by using $" \psi-\$$ pumping,"70 which is directly analogous to the helicity-injection method previously proposed for RFPs termed "F- $\theta$ pumping."71-73 The phased, sinusoidal injection of magnetic helicity has yet to be fully verified experimentally, although initial evidence is encouraging. Furthermore, a theoretical base can be developed by assuming a strong coupling between toroidal and poloidal circuits through the plasma, a coupling that is typically exhibited both in $\operatorname{RFP}^{4,42}$ and spheromak ${ }^{4-48}$ experiments. Using an inductive configuration similar to the S-1 experiment at $\mathrm{PPLL}^{48}$, which is illustrated in Fig. 2.1.-6, requires closed magnetic flux along with plusma to encircle the large inductive toroidal core that surrounds the final p.asma configuration and is used to initiate the plasmoid. Protection of the flux core against sputtering from the bulk plasma presents engineering uncertainties. Since this large electrically insulated flux core must reside inside or close to the main plasma chamber, serious geometric problems arise when attempting to impose a close-fitting conducting shell and maintaining plasma equilibrium. Both equilibrium and stability problems have impeded experimental progress for this configuration, requiring further effort to include a conduction shell as close to the plasma as is practical. Requiring a large inductive flux core to be situated around the plasmoid and inside the vacuum chamber also creates difficulties for the reactor. The entire plasma system necessarily becomes much larger in order to accommodate the toroidal flux-core. Also, the close proximity of this flux core to the plasma would require special considerations of neutron damage and nuclear heating. In summary, the preliminary experimental results and problems of an inductively produced and/or driven spheromak have directed the present study towards the stationary, gun-driven configurations.

The potential for a steady-state gun-driven spheromak reactor was first suggested ${ }^{8}$ using helicity injection principles based upon the Taylor theory of near-minimum-energy states.43,44 Since proposing this technique, encouraging experimental results that have verified this conjecture have been generated by the Los Alamos group, with the spheromak plasma being produced and refluxed using a continuously driven plasma gun. ${ }^{74}$ Internal magnetic flux is provided by driving the gun electrodes after the configuration is formed.: In fact, the internal flux can be increased using this technique, wherein a plasma of outside radius $R=0.40 \mathrm{~m}$ and height $\mathrm{L} / 2=0.2 \mathrm{~m}$, as shown in Fig. 2.1.-7, is maintained at a temperature of $\sim 30 \mathrm{eV}$ as the poloidal magnetic flux is increased over a 


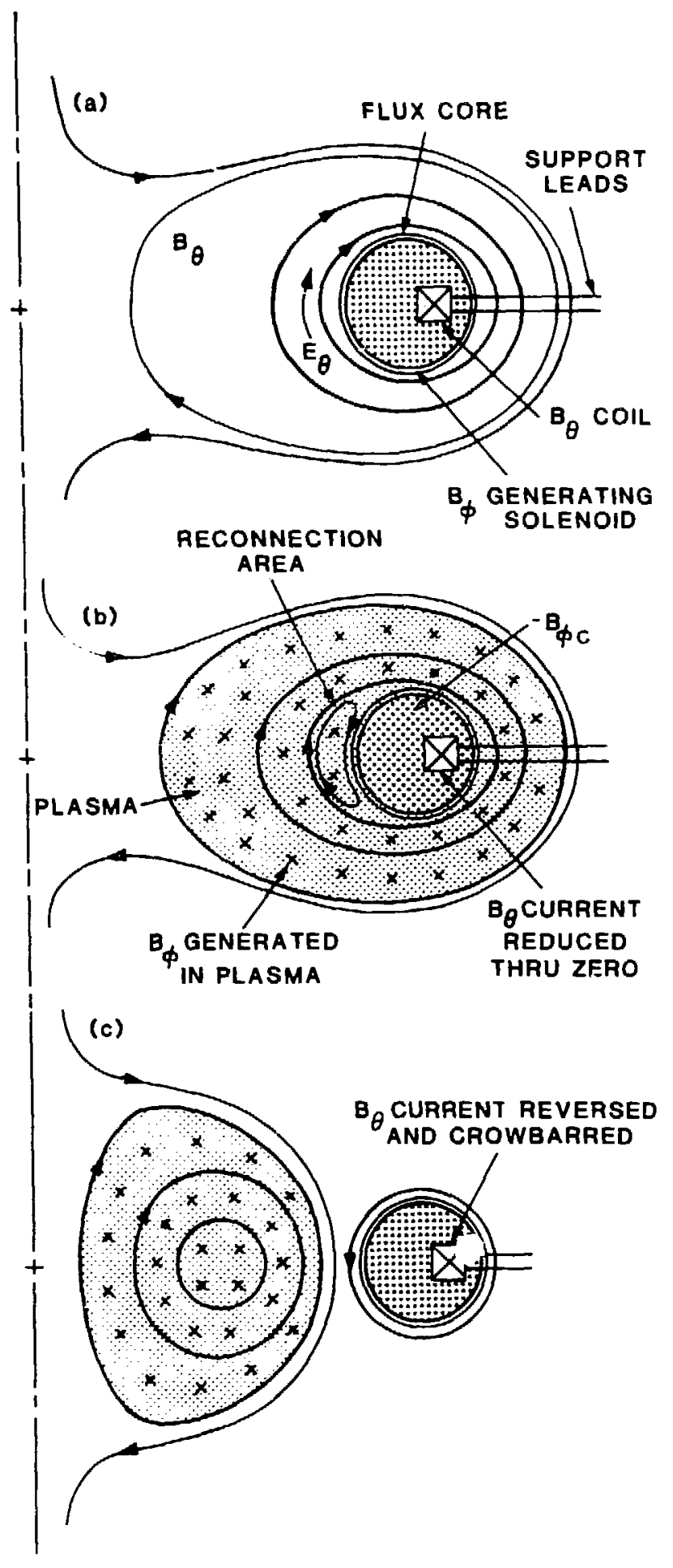

Fig. 2.1.-6. Inductively produced spheromak configuration S1 at PPPL. 48,49 

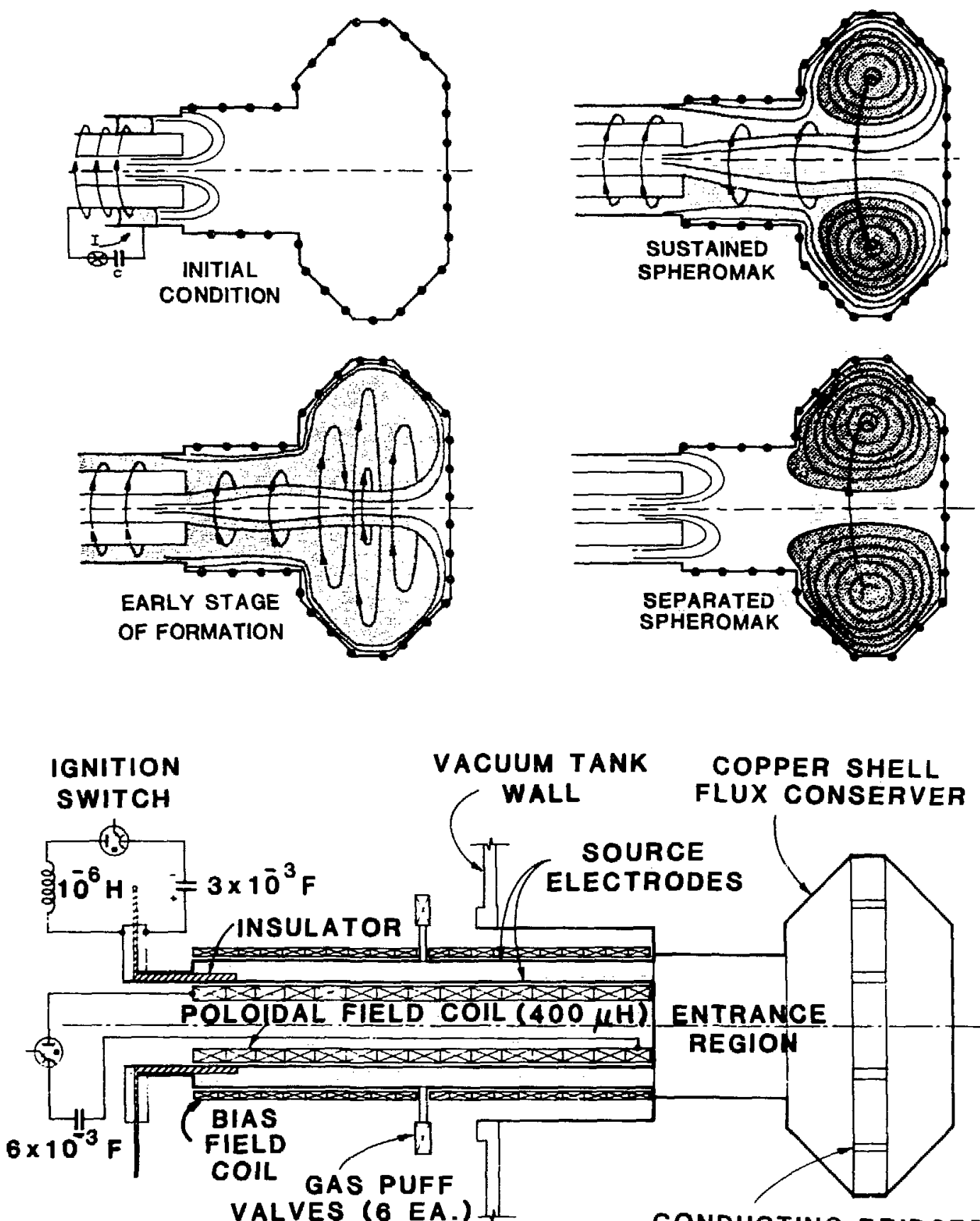

VACUUM TANK

WALL

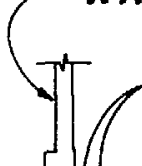

SOURCE ELECTRODES
COPPER SHELL FLUX CONSERVER

VALVES ( 6 EA.) I

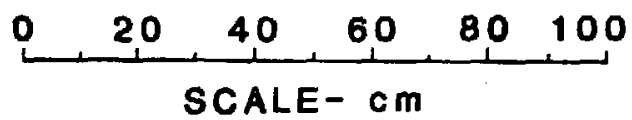

Fig. 2.1.-7. Experimental Los Alamos CTX spheromak experiment showing sustained and separated operation. off-axis electrode systems have also been operated successfully. 75 
period of time $(\sim 5 \mathrm{~ms})$ an order of magnitude longer than the resistive decay time for that configuration. Since this flux drive involves the direct contact of open magnetic surfaces or divertor current-drive flux lines on electrode plates, impurity influx is a major concern for the experimental technique and for any reactor embodiment based thereon. Completely separating the flux surfaces from the electrode drive (Fig. 2.1.-7) yields an independent spheromak which has achieved temperatures of $\sim 100 \mathrm{eV}$ at betas of 0.1 (Table 2.1.-I).

A stationary, electrode-driven spheromak satisfied stability considerations by positioning a closely fitting conducting shell around the spheromak; this configuration is also consistent with the goal of a compact fusion reactor. Considering the experimental verification of steady-state helicity injection adds impetus to the study of this concept as a potential fusion reactor. Early suggestions, ${ }^{68}$ as illustrated in Fig. 2.1.-8, ongoing design studies, 69 and the present design-definition study, all seek to identify the major characteristics of a compact spheromak reactor (CSR), explore the major subsystem requirements, and specify the required physics performance of the spheromak plasma and helicity-injection mechanism, while addressing related issues of impurity control.

\subsection{Approach}

2.2.1. Overview. The overall study approach used to define a compact spheromak reactor (CSR) is similar to the scenario developed for the Compact Reversed-Field Pinch Reactor (CRFPR).25,26,76 The major tool for exploration of CSR designs is an appropriately adapted version of the CRFPR systems code. In this model physics, engineering, and economics considerations are closely coupled to allow a comprehensive survey of reactor designs using the cost of electricity (COE, mills/kWeh) as the main object function. In addition to a specific cost-optimized system, this design formalism also defines an entire spectrum of devices based upon minimum COE that could be built, depending on a specific physics-based plasma transport scaling; the cost-driven system analysis itself is independent of the specific transport scalings once the minimum-cost neutron wall loading is chosen for a given net electric power.

The framework for the CSR optimization approach is described in Table 2.2.-I. The listed input parameters include system characteristics that are broadly delineated according to physics, engineering, and economics. Input parameters include subsystem characterizations that are generally determined 


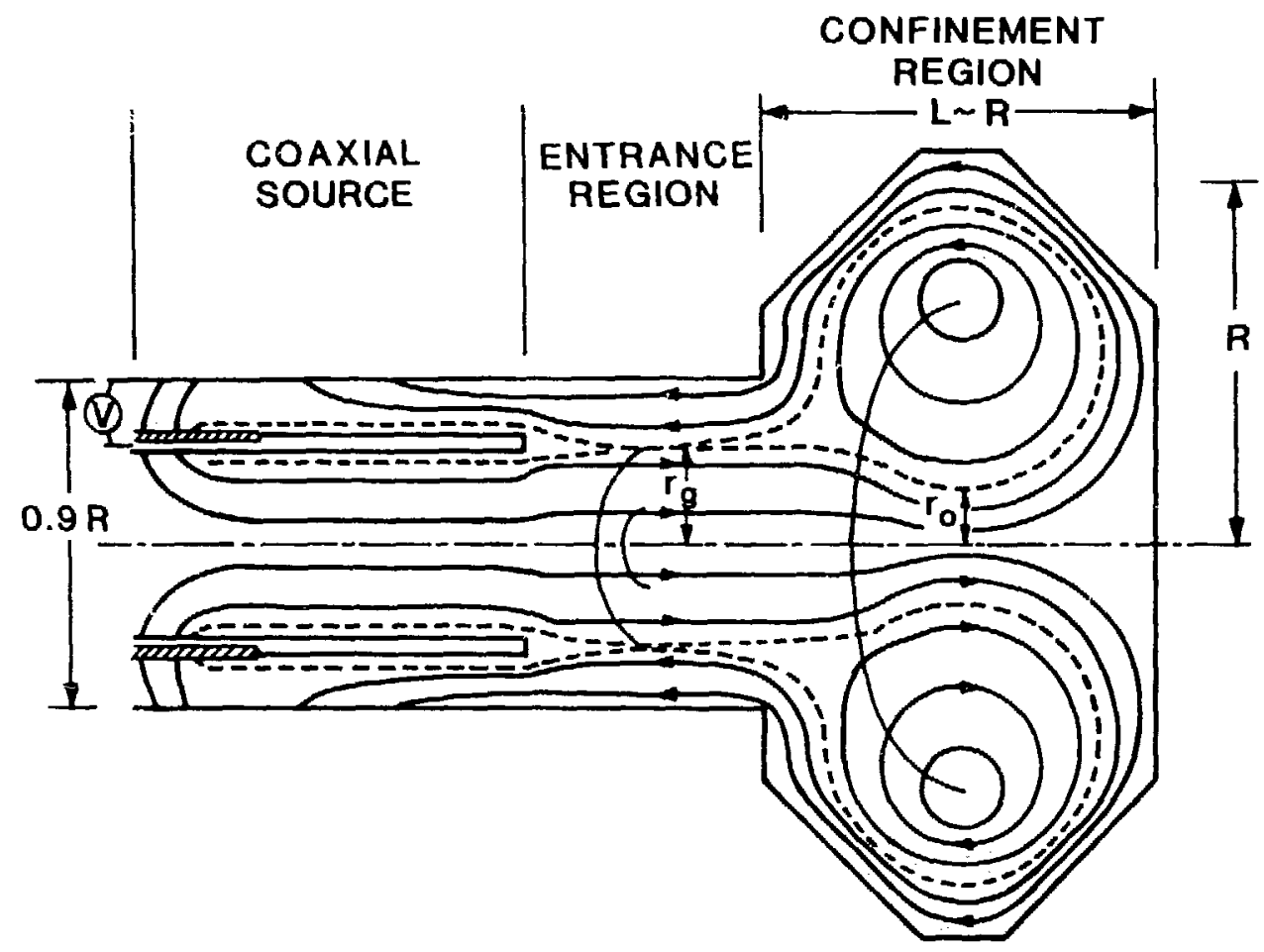

Fig. 2.1.-8. Suggested geometry for a steady-state spheromak reactor and experimental refluxing verification from the Los Alamos CTX experiment. Other electrode configurations have recently been suggested and experimentally verified ${ }^{5}$ wherein off-axis electrodes remove the electrode/direction surfaces out of the direct line of sight of the spheromak plasma.

from experiment or simply are defined as input. Experimentally derived variables include plasma equilibrium, beta, and plasma profiles, along with plasma-gun/helicity injection characteristics. The plasma equilibrium and magnetics properties are specified using a two-dimensional model described in Sec. 3. Engineering and economics drivers, such as blanket thickness, first-wall lifetime, and unit costs, must also be externally provided. Finally, the reactor designs must specify the fusion fuel and net electric power. All of the aforementioned quantities can be specified within an accuracy range, although substantial uncertainty may exist. Sensitivity analyses are performed to identify those systems that require better definition. 
SUMMARY OF CSR OPTIMIZATTON APPROACH EXPRESSED AS

INPUT VERSUS DERIVED PARAMETERS

MODEL INPUT

- Plasma equilibrium

- Beta

- Plasma temperature/density profiles

- Gun helicity injection efficiency

- Fuel

- Net electric power

- Blanket thickness

- Blanket energy multiplication

- Plasma-gun characteristics

- Magnetics

- Unit costs

- Construction time/costs/schedule

- First-wall radiation lifetime

- Plant factor algorithm
MODEL DERIVED

\section{PHYSICS}

- Plasma operating temperature/density

- Aspect ratio

- Minor plasma radius

- Plasma transport

\section{ENGINEERING}

- Engineering Q-value

- First-wall neutron loading

- Coil thicknesses

- Normal/superconducting coils

\section{ECONOMICS}

- Plant factor

- Unit direct cost

- Cost of electricity

2.2.2. Physics. The optimal plasma operating temperature, aspect ratio, minor p’asma radius, or plasma particle and energy transport are not known a priori. These variables are determined to minimize the COE. The economically optimal plasma transport that results from this exercise can then be reconciled with various transport scalings that are theoretically or experimentally derived for the spheromak configuration. Importantly, therefore, this optimization formalism specifies the minimum required plasma containment properties, below which engineering problems may arise as a result of excessive first-wall thermal neutron loading generated by the higher density needed to assure an ignited plasma.

The optimization procedure described above is also used to evaluate choices between normal versus superconducting coils, coil thickness for resistive magnets, engineering $Q$-value $\left(Q_{E}\right.$, inverse of electric recirculating power fraction), and first-wall neutron loading. Economic (i.e., COE) optima usually 
do not occur at the point of maximum $Q_{E}$. Finally, the plant factor, $p_{f}$, is derived from scheduled and unscheduled downtimes, including the need to replace the first-wall/blanket after a specified neutron fluence. These compact system designs investigate integral plasma containment chambers which are replaced as a single unit in a time of approximately one month; the plant factor, therefore, depends on the neutron wall loading, wall life and replacement time.

The options and choices for the systems code input summarized in Table 2.2.-I are expressed in Table 2.2.-II. For convenience, major references

TABLE 2.2.-II

SUMMARY OF SOURCES USED TO GENERATE OR PROVIDE THE CSR SYSTEM CODE INPUT

PHYSICS

REFERENCE

Plasma magnetic-field equilibria (plasma power weighting functions)

-zero beta analytic (Bessel functions/sinusoids)

$77-80$

-finite beta analytic, $p \propto \psi^{2}$, coulomb-wave functions 81-83

-numerical solution for arbitrary $j$ and $B \quad T B D$

Beta

$58,84,85$

Plasma $\mathrm{T}, \mathrm{n}$ and $\psi$ profiles

$58,84,85$

Plasma gun/helicity injection

-experimental input, analytic approximation

$58,84,85$

-divertor calculation, 3-D magnetics/ZEPHYR

86 (not used)

\section{ENGINEERING}

Magnetics (coil location/characteristics)

-analytic from Shafranov formulas (questionable for spheromak) 87-89

-numerically enforced analytic plasma equilibrium 90

-3-D magnetics

TBD

Thermal-cycle efficiency, auxiliary power requirements

26,76

Blanket thickness (neutron multiplication)

26,76

Plasma-gun characteristics

$58,84,85$

\section{ECONOMICS}

Unit costs

Construction time/costs/schedule

$4,20,21$

wall lifetime

$4,20,21$ 
are noted in Table 2.2.-II for each category. Specifying the equilibrium configurations determines plasma profiles and plasma-power (fusion, transport, radiation) weighting functions. Determination of the equilibrium may be accomplished using zero-beta analytic solutions,77-80 finite-beta analytic

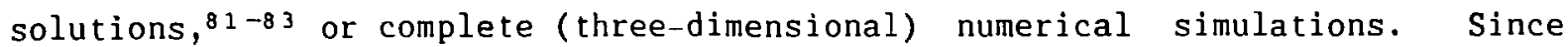
the necessary plasma-profile weighting functions are not strongly affected by finite beta, the zero-beta analytic formulation has been chosen. The ideal solutions (i.e., Bessel-function in radius, sinusoidal in height) require large plasma current density at the plasma separatrix, which is consistent with the notion of helicity injection from the plasma edge (Sec. 3.). Considering the uncertainty in the determination of the plasma profiles in present-day spheromaks, a two-dimensional numerical solution for arbitrary current density and magnetic field is judged as inappropriate at this time; the necessary transport information as a function of geometry and magnetic parameters is simply not available. Nonetheless, the expressions based on zero-beta analytic equilibria have proved to be a powerful means to predict experimental behavior, 84 and are expected to represent accurately the reactor configuration. This equilibrium formulation specifies the magnetic field and current density, while the plasma pressure profile is experimentally inferred to be proportional to the magnetic flux function.

The plasma-gun characteristics are derived directly from present experimental observations and extrapolations. Matching of the gun parameters and refluxing (current-drive) properties to the plasma requirements represents one of the greatest uncertainties in the system optimization and design. This matching is performed by analytically joining the plasma-gun, spheromak plasma, and the connecting open-field-line divertor regions; all regions are treated separately and are connected by appropriate interface conditions. This three-region system could be appropriately modeled by a three-dimensional magnetics model that consistently couples the current-drive source to the plasma. The open-field-line surface surrounding the spheromak plasma and connecting to the plasma gun would need be modeled by an appropriate divertor-surface code. ${ }^{86}$ Interfacing such detailed models with a general systems code would be difficult at best. Fortunately, the study results exhibit little sensitivity to the temperature profile within the divertor surface, indicating the adequacy of the analytic expression derived for the temperature distribution along the separatrix/divertor surface that carries currents 
provided by the gun electrodes. Resistive power consumption associated with these currents is derived by numerical integration along the flux channel connecting the gun-electrode with the spheromak, using the analytic divertor flux channel temperature profile. The major uncertainties arise when considering the efficiency of poloidal flux injection by the plasma gun; these processes are assumed to obey the Taylor concept ${ }^{4,44}$ of minimizing the magnetic energy per unit helicity. To model this process theoretically would require a complete three-dimensional MHD calculation of the full system and is beyond the scope of this study. In lieu of this information, the flux injection efficiency is treated as a design variable subjected to sensitivity analysis and compared to present experimental observation.

2.2.3. Magnetics. Establishing a steady-state plasma equilibrium requires the existence of an externally produced vertical magnetic field. The definition of this engineering subsystem may be accomplished using several methods (Table 2.2.-II), each having varying degrees of accuracy. The analytic Shafranov formulas are commonly used with a high-degree of success in RFP or tokamak configurations. The low aspect ratio of the spheromak, however, reduces the accuracy of the solution. A full three-dimensional equilibrium calculation, however, is not consistent with the analytic plasma equilibrium used and would be difficult to implement in a general systems code, where many cases must be computed in the course of a cost optimization. The steady-state plasma equilibrium, therefore, is enforced in the systems code by using the analytic plasma equilibria. Discretizing the analytically derived toroidal plasma current into a two-dimensional mesh of hoop currents permits the flux distribution to be calculated directly using elliptical integrals.90 This analytic equilibrium presumes a perfectly conducting shell located at the separatrix, which in the present case can be represented by conductors positioned outside the blanket/shield region. These hoop conductors are located along a predefined surface and must carry a sufficient current to locate the separatrix at the predefined plasma radius. This method of enforcing plasma equilibria and determining magnet coil currents and locations can be executed relatively rapidly while retaining an acceptable level of accuracy. 
2.2.4. Engineering. In addition to specifying the equilibrium magnet coils, the neutron-moderating blanket, thermal-cycle efficiency, and auxiliary power requirements are largely derived from the CRFPR design experience,26,76 (i.e., Li ${ }_{17} \mathrm{Fb}_{83}$-cooled blanket and a water-cooled first-wall and limiter), although these characteristics must be carefully weighed when applied to the CSR considering the geometry differences. In particular, the CSR has the potential to operate with a natural divertor (i.e., the plasma-gun electrodes), which automatically transports the charged particles to a surface that is exterior to the plasma chamber. In addition, all PbLi coolant flows parallel to the magnet field in the CSR, enhancing the cooling abilities of the liquid metal for a given pumping power and blanket pressure. Such a system may avoid the requirement for a high-performance water-cooled first-wall or plasma-limiter surface. The CRFPR design 6 dealt with the power shed by the plasma (ohmic and alpha-particle power) using in situ water-cooled pumped limiters which, in addition to a water-cooled first wall, degraded the neutron-breeding characteristics of the liquid-metal blanket, increased the blanket thickness, and generally reduced the overall thermal-conversion efficiency. A purely liquid-metal-cooled first-wall/blanket structure for the CSR, therefore, should allow somewhat thinner first wall/blanket structures, although the plasma-gun electrode/divertor surfaces may still require water cooling (albeit, located external to the main plasma chamber), leading to somewhat degraded thermal-cycle efficiencies compared to a system that was cooled only with liquid metal. In view of the preliminary nature of the CSR design definition, the aforementioned engineering parameters are inferred from the CRFPR design with major subsystem uncertainties subjected to sensitivity analyses.

2.2.5. Economics. With the physics performance and required engineering subsystems defined, a complete economics analysis must be performed to evaluate the COE. The economics model follows the magnetic fusion energy (MFE) costing guidelines used in the STARFIRE tokamak ${ }^{4}$ design, after accounting for important exceptions. This costing formalism assumes a "tenth-of-a-kind" plant, which is appropriate for most of the balance of plant, but may be unrealistic for the FPC. Unit costs for the first wall, blanket, shield, and magnet coils are based upon present-day expectations, leading to unit costs that are at least a factor of two greater than the MEE costing guidelines ${ }^{2}$ as originally used in STARFIRE. ${ }^{4}$ The construction time, cost, and schedule are presumed similar to the 
STARFIRE design, ${ }^{4}$ which for the CSR should be correspondingly ontimistic considering the physically smaller system and a greater opportunity for off-site factory fabrication. Finally, the first-wall neutron lifetime directly impacts the downtime and plant factor, which adversely affects the COE. This important quantity represents a major unknown for fusion engineering in general, although values in the range $\dot{5}-15 \mathrm{MWyr} / \mathrm{m}^{2}$ for stainless steels are expected. Since high-heat-flux systems typically invoke materials other than stainless steel (copper or vanadium alloys), this variable becomes even less well defined. Parametric analysis must then be performed over the expected range of firstwall/blanket lifetimes.

2.2.6. Systems. The systems studies code used to mod?l the CRFPR 25 has been amended to model the spheromak reactor configuration illustrated in Fig. 2.2.-1. The CSR plasma of height, $L$, and outside radius, $R$, is contained in a spherical vessel surrounded by a neutron moderating blanket, $\Delta b$, and equilibrium-fieid coil (EFC) of thickness, $\delta_{c}$; no toroidal-field coils (TFCs) are required. Plasma current drive would be provided by a co-axial plasma gun, which also initiates the plasma and would serve as a plasma divertor. A logic diagram for the systems analysis is given in Fig. 2.2.-2, which shows the optimization sequence as well as the separation between input versus derived quantities (Table 2.2.-I). Desired net electric powers, $P_{E}$, and the plasma aspect ratio, $A=L / R$, of the spheromak plasma are input parameters. For convenience, a cylindrical conducting shell is presumed to represent the inside of the spherical vessel. This approximation is justifiable because the "corners" of the cylindricalized equilibrium configuration shown in Fig. 2.2..1 have little effect on the overall system. The aspect ratio is typically optimized, while the net-electric power must be specified as input. With the aspect ratio given, the analytic equilibrium can be defined, $\left(\mu_{0} \vec{j} / \vec{B}=\right.$ CONSTANT $)$, as given by the Taylor minimum-energy theory and described in Sec. 3.1. Upon giving the DT reactivities and plasma (temperature/density) profiles, a two-dimensional integration of all plasma powers (alpha-particle, radiation, and ohmic-heating powers, Sec. 3.1.) over the plasma volume yields the weighting functions used to express volumetric powers in terms of an average plasma density and temperature. This calculation also leads to the $n \tau_{E}$ value required for ignition for the sperified plasma profiles and temperature. These weighting functions, $g_{i}$, and the ignition condition, $n \tau_{E}$, are dependent only on the aspect 


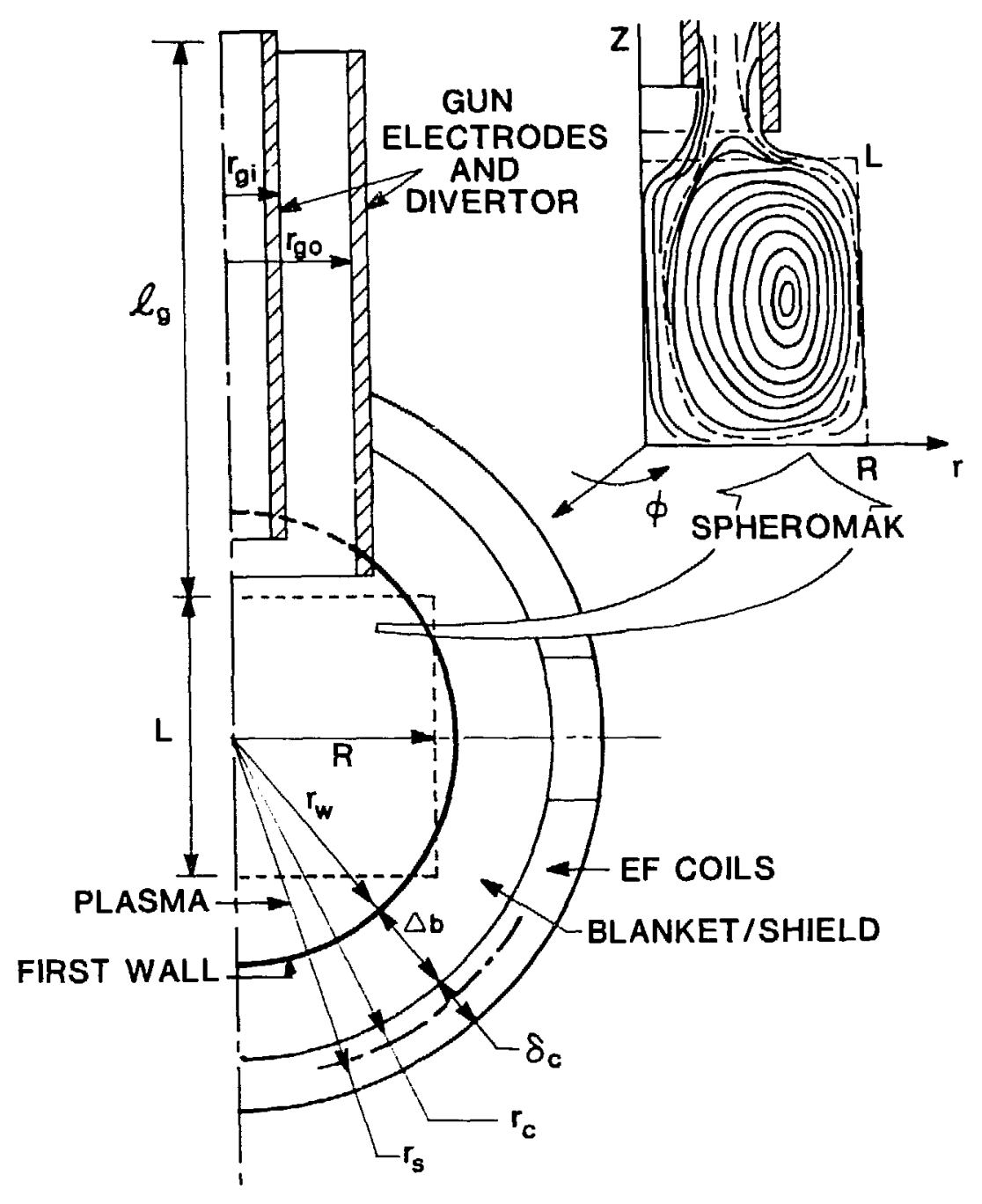

Fig. 2.2.-1. Schematic diagram of CSR fusion-power-core geometry used in the parameters systems model, along with a cylindrical representation of the spheromak plasma that gives an accurate and convenient analytical equilibrium model.

ratio, $A$, and need not be redefined for the remaining calculation or changes in $\mathrm{P}_{\mathrm{E}}$.

The plasma radius, $R$, is then varied over a specified region, with the total thermal power divided by the density squared, $\mathrm{P}_{\mathrm{TH}} / \mathrm{n}^{2}$, or neutron wall loading divided by density squared, $I_{W} / n^{2}$, being computed given the appropriate plasma power weighting functions, plasma temperatures, DT reactivities, and the neutron-moderating blanket sergy multiplication, $\mathrm{M}_{\mathrm{N}}$. For a given geometry the 


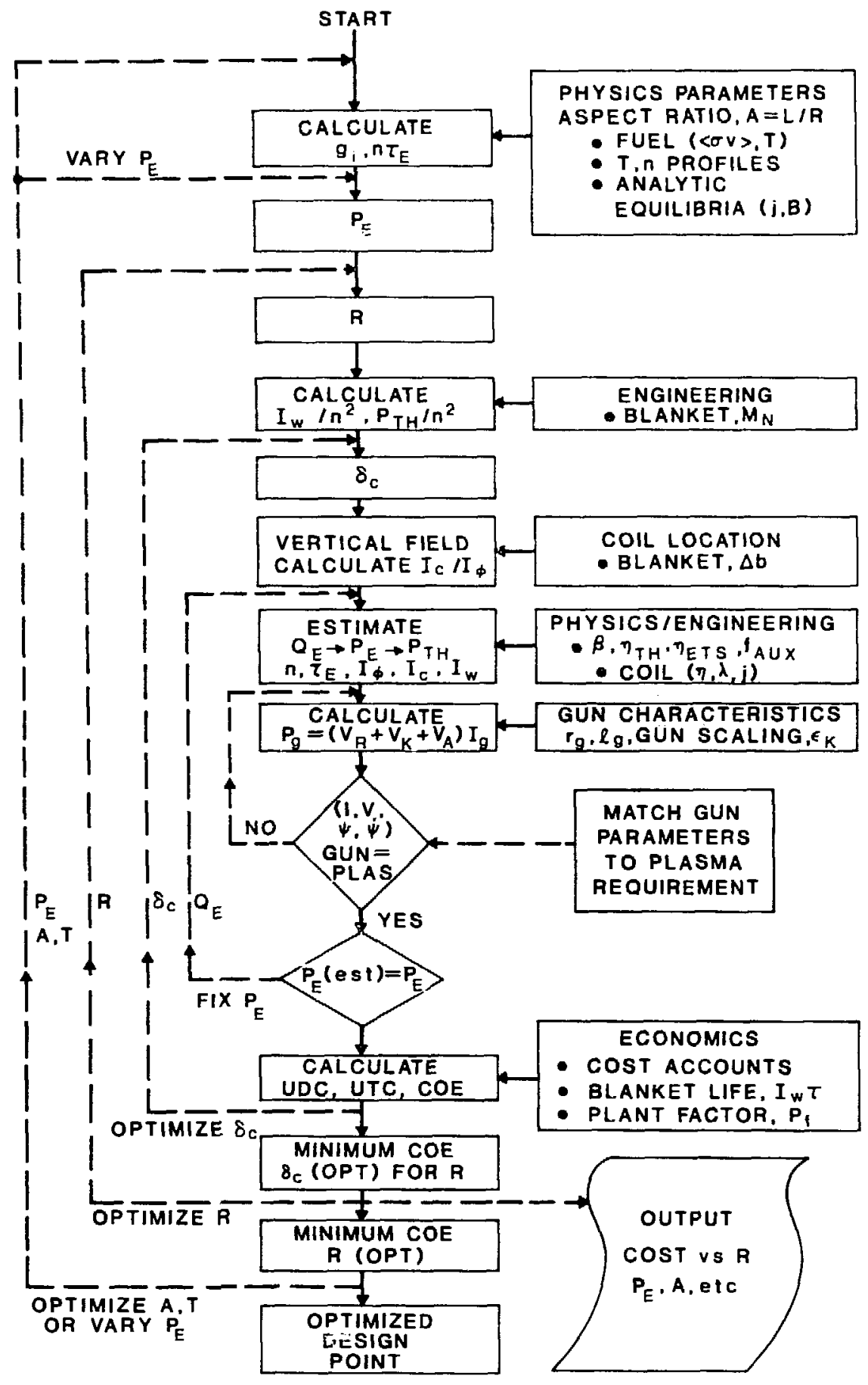

Fig. 2.2.-2. Logic diagram for CSR systems optimization showing inputted, varied, and derived parameters. 
total thermal power output can be calculated for a specified density at this stage of the algorithm shown in Fig. 2.2-2.

The next parameter variation surveys various EFC thicknesses, $\delta_{c}$, wherein the capital cost of conductor is balanced against the effective cost of electric power recirculated to a normal-conducting coil. Having defined particular coil and blanket thicknesses specifies the radial position, $r_{c}$, of the equilibriumfield coils. Locating this coil over the region illustrated in Fig. 2.2.-1 defines the ratio of coil current to plasma toroidal current, $I_{c} / I_{\phi}$ as explained in Sec. 3.4. Upon calculating the plasma current from pressure balance, the necessary EFC current and the associated resistive losses are determined.

Since the total electrical recirculating power fraction, $1 / Q_{E}$, is not known a priori, an initial guess must be made. Using the specified $P_{E}$, the total thermal power, $\mathrm{P}_{\mathrm{TH}}$, is estimated from $\mathrm{P}_{\mathrm{TH}}=\mathrm{P}_{\mathrm{E}} / \mathrm{r}_{\mathrm{TH}}\left(1-1 / \mathrm{Q}_{\mathrm{E}}\right)$, where the overall system thermal-conversion efficiency is $\eta_{\mathrm{TH}}$. The required thermal power is now used to specify the operating plasma density using the previously deterinined ratio, $\mathrm{P}_{\mathrm{TH}} / \mathrm{n}^{2}$.

Recalling that the ignition condition, $n \tau_{\mathrm{E}}$, has already been calculated for the assumed profiles, having the density establishes the necessary energy confinement time, $\tau_{E}$, for this particular reactor system. With the plasma beta as input the plasma magnetic field and current levels are determined, which, as previously noted, are then directly coupled to the external equilibrium coils via the $\mathrm{I}_{c} / \mathrm{I}_{\phi}$ ratio.

The recirculating electric power fraction, $\varepsilon=1 / Q_{E}$, must be recalculated using all available power needs, including resistive power to the EFCs, electric power to the plasma gun, $P_{g}$, energy-transfer efficiency, $\eta_{E T S}$, and plant auxiliary power, $\mathrm{P}_{\mathrm{AUX}}=\mathrm{f}_{\mathrm{AUX}} \mathrm{P}_{\mathrm{ET}}$, as explained in Sec. 3.4. All of these quantities have been redefined at this point in the computation, except for the required input power to the plasma gun. The main purpose of the helicity-injecting plasma gun is the refluxing of the plasma resistive current or helicity decay, which requires a power, $V_{K} I_{g}$, to replace the plasma ohmic losses with an efficiency, $\varepsilon_{K}$, for a divertor-channel current of $I_{g}$ and a helicity injection voltage of $V_{K}$. Driving this divertor current also incurs a resistive channel loss associated with the roltage, $V_{R}$, and may require the incorporation of a loss associated with the arc voltage drop, $V_{A}$, at the electrode. All of the charged-particle power is assumed to be diverted to the 
plasma gun/electrode and is used to define analytically the divertor channel temperature distribution ( $\mathrm{Sec}$. 3.3.). The temperature distribution is used to find the resistive loss associated with the divertor current flow, $\mathrm{I}_{\mathrm{g}}$. This procedure is complicated by the need to match a specific (experimentally derived) relationship between the gun voltage, $V_{g}$, current, $I_{g}$, input flux, and helicity-injection properties to the similar variables required to sustain the spheromak plasma. This interfacing requires an additional iterative loop, as is shown in Fig. 2.2.-2, which iterates on the fraction of poloidal flux needed to be diverted from the plasma into the plasma gun.

Having calculated the necessary recirculating electric power, $\mathrm{P}_{C}=(1-\varepsilon) \mathrm{P}_{\mathrm{ET}}$, the resulting net electric power is compared to the value originally specified, $\mathrm{P}_{E}$. Using the new value of $\mathrm{Q}_{E}$, a better estimate of the thermal power, ${ }{ }_{T H}$, is obtained iteratively until the input value of the netelectric power is achieved. Since the economics model is a function of the net electric power, subsystem definition on an economic basis must be performed using systems of equal net electric output.

With the specified CSR generating the desired net electric, ${ }_{E}$, the compilation of a complete systems economic analysis using the magnetic fusion energy cost accounting is performed. The unit cost accounts yield the unit direct costs, UDC $[\$ / \mathrm{kWe}(n e t)]$, unit total cost, UTC (\$), and, finally, the COE (mills/kWeh) after specifying the first-wall/blanket lifetime, which in turn dictates the plant factor for a given neutron wall loading. Varying the coil thickness, $\delta_{c}$, exhibits a $\mathrm{COE}$ minimum as the capital cost of increasing conductor thickness is traded for the decrease in electrical recirculating power. Selecting the coil thickness that yields a COE minimum, the optimization process continues as the plasma radius, $R$, is varied. Typically, the radius variation also exhibits a minimum for a fixed $P_{E}$, with lower radii forcing excessive neutron wall loadings (shorter lifetimes and decreased plant factor) and lower $Q_{E}$ while large radii systems have substantially higher masses (and capital. costs) and once again lower $Q_{E}$. At this point in the optimization procedure (bottom of Fig. 2.2.-2), all major variables are plotted as a function of $R$ with an extensive tabular parameter and cost summary only printed at the minimum-COE value of the outside plasma radius. This cost-optimized design point is then generated for a range of net electric powers, subject to an aspect-ratio optimization. As previously discussed, an important result of this procedure is the derivation of the required energy confinement time, $\tau_{E}$ (OPT), 
versus $R$ to achieve the economically optimal design. This confinement time is compared to other experimental and theoretical confinement scalings to delineate which designs are accessible from the viewpoint of confinement scaling. 
3. PARAMETRIC SYSTEMS MODEL

\subsection{Equilibrium Magnetics}

The spheromak is a toroidal axisymmetric configuration in which the primary confinement field, $\mathrm{B}_{\theta}$, is poloidal and is generated by a toroidal current, $I_{\phi}$, flowing in the plasma, as is illustrated in Fig. 3.1.-1. The spheromak plasma supports a strong internal toroidal bias field, $B_{\phi}$, to stabilize sausage $(m=0)$ and elliptical $(m=2)$ distortions, althrugh the external toroidal field is zero. Grossly unstable MHD kink modes $(m=1)$ with wavelengths longer than the minor plasma radius, $r_{D}$, are stabilized by a close-fitting conducting shell. Because the mechanical structure is positioned within the center of the torus, the spheromak is a nember of the compact corus family, which also includes the field-reversed theta pinch $\left(B_{\phi} \simeq 0\right)$, as is illustrated in Fig. 2.1.-3. The unique topology of these plasmoids permits translation from the source region to a plasma burn chamber, typically requiring the continuous presence of a conducting shell surface.

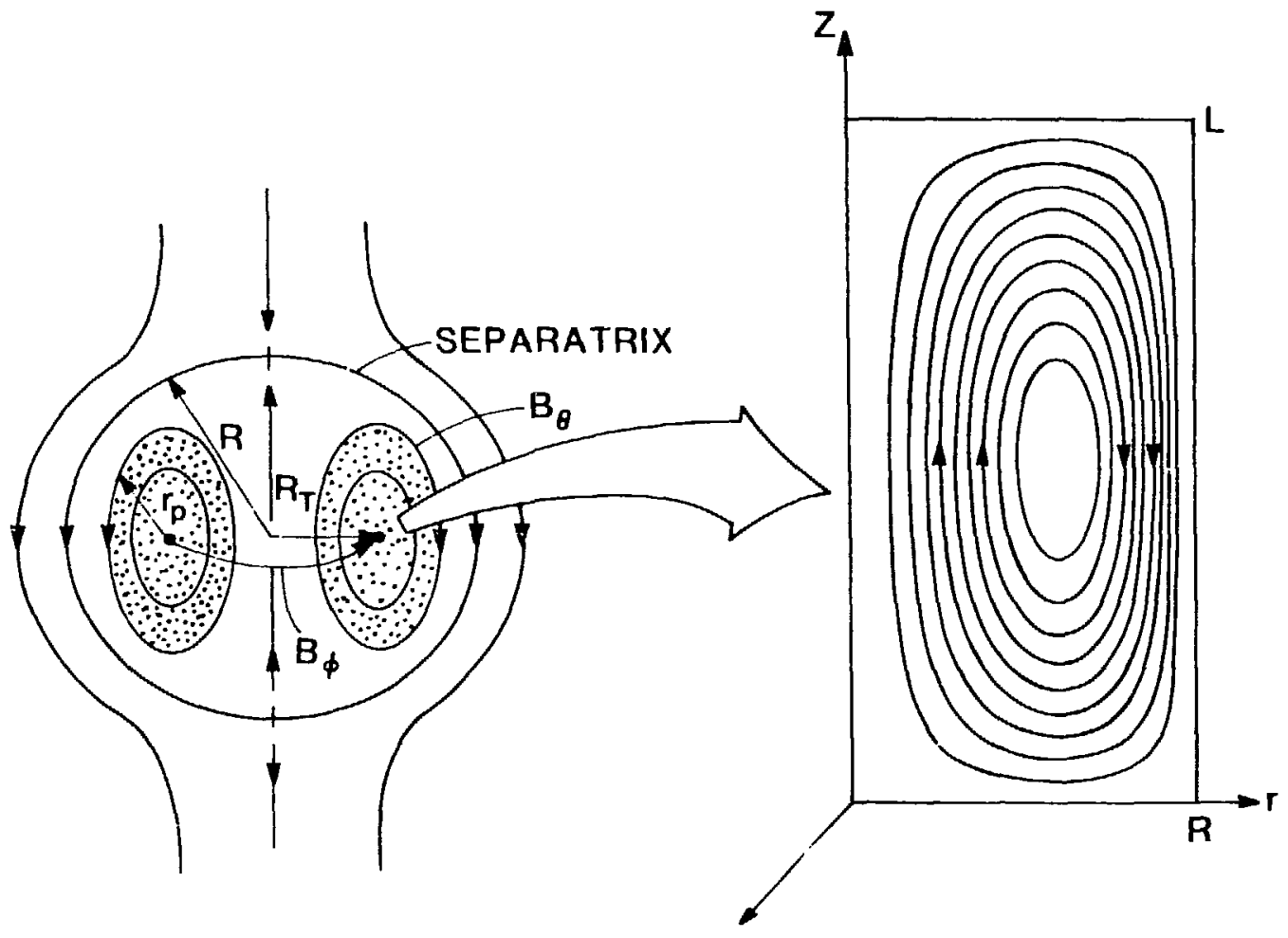

Fig. 3.1.-1. Spheromak plasma configuration showing the major toroidal radius, $\mathrm{R}_{\mathrm{T}}$, minor radius, $\mathrm{r}_{\mathrm{p}}$, and separatrix radius, $\mathrm{R}$. 
The existence of spheromak equilibrium configuration is based on a theory of relaxed plasma states, as proposed by Taylor.43,44 This theory proposes a near-minimum-energy state, wherein the plasma maintains a nearly constant average magnetic helicity, $\mathrm{K}$,

$$
K \equiv \int_{V_{p}} \vec{A} \cdot \vec{B} d V_{p}
$$

over the volume, $V_{p}$, where $\vec{A}(\vec{B}=\vec{\nabla} \times \vec{A})$ is the magnetic vector potential. Given any arbitrary dissipation mechanism, the Taylor theory predicts that a plasma surrounded by a flux-conserving shell relaxes to a near-minimum-energy state characterized by $\mu=\mu_{0} \vec{j} \cdot \vec{B} / B^{2}$ equal to a constant, implying all plasma currents are flowing parallel to the magnetic field (i.e., a force-free, $\overrightarrow{\nabla p}=0$ configuration). This force-free state provides the basis for spheromak equilibrium configurations. Experiments have verified that these equilibria also support plasma pressure, requiring only small profile modification from the idealized force-free $(\beta=0)$ state.

Analytic spheromak equilibria have been formulated for several geometries, including nearly spherical shapes $80,82,83$ or assuming a cylindrical conducting shel177-79,81 of height, L, and outside shell radius, R; Fig. 3.1.-1 illustrates this latter approximation. Because stable configurations typically require ${ }^{6}$ oblate geometries ( $L / R \leq 2)$, the cylindrical model is a good representation of most experimental machines and is expected to reflect accurately the reactor configuration. This representation is even better than expected, since only a small amount of plasma current exists in the "corners" of the cylindrical-shell model.

Equilibrium configurations have also been analytically derived for zero pressure $77-80$ and various finite pressure, profiles, including $p(r) \propto \psi(r)^{82,83}$ and $p(r) \propto \psi^{2}(r),{ }^{81}$ where $\psi(r)$ is the poloidal flux. Adding pressure to the model shifts the flux surfaces outward, although the effect is not pronounced; in the cylindrical geometry the center of the plasma or point of maximum poloidal magnetic flux occurs at $0.628 \mathrm{R}$ for zero beta and moves outward to $0.707 \mathrm{R}$ at the maximum theoretical beta of $0.45 .{ }^{84}$ Even this large beta range has little effect on the plasma-profile weighting functions, which reflect volumetric plasma properties such as resistive current decay, radiation and transport loss, and alpha-particle heating (Sec. 3.2.). Since maximum betas of 
$\leq 0.2$ are typically considered in this analysis, no benefit is expected if a Finite-beta formulation is used (i.e., $p \propto \psi^{2}$ ) yielding Coulomb wave functions. ${ }^{81}$ Once again, the added complication of using more detailed analytic formulations is not warranted.

Using the coordinates given in Fig. 3.1.-2, a zero-pressure plasma equilibrium is represented by the magnetic field functions as follows:

$$
\begin{aligned}
& B_{r}=-B_{0}\left(k_{z} / k_{r}\right) J_{1}\left(k_{r} r\right) \cos \left(k_{z} z\right) \\
& B_{\phi}=B_{0}\left(1+k_{z}^{2} / k_{r}^{2}\right)^{1 / 2} J_{1}\left(k_{r} r\right) \sin \left(k_{z} z\right) \\
& B_{z}=B_{0} J_{0}\left(k_{r} r\right) \sin \left(k_{z} z\right),
\end{aligned}
$$

where the radial, toroidal, and vertical magnetic fields $B_{r}, B_{\phi}$, and $B_{z}$, respectively, consist of Bessel functions of the first kind, $\mathrm{J}_{0}$ and $\mathrm{J}_{1}$, along with sinusoidal functions. The peak (on-axis) magnetic field, $B_{0}$, is yet to be determined. The key system eigenvalues are then defined as follows:

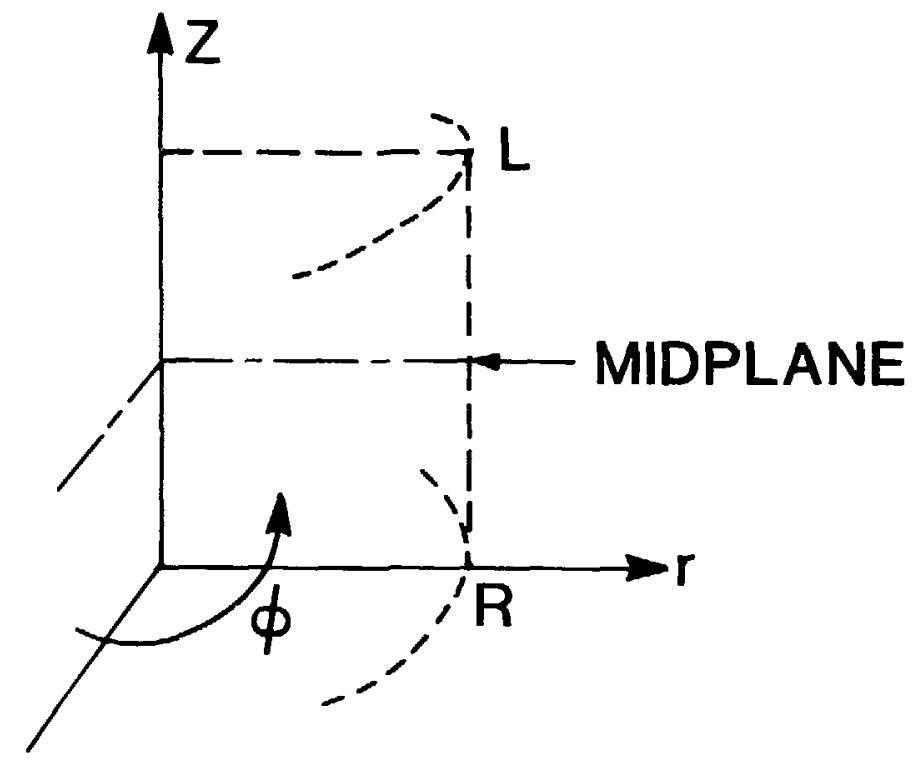

Fig. 3.1.-2. Coordinate system used to define the cylindrical-shell cSR model. Midplane is located at $\mathrm{Z}=\mathrm{L} / 2$. 


$$
\begin{aligned}
& k_{r}=a_{11} / R \\
& k_{z}=\pi / L \\
& k=\left(k_{r}^{2}+k_{z}^{2}\right)^{1 / 2},
\end{aligned}
$$

where

$$
\begin{array}{ll}
J_{0}\left(a_{01}\right)=0 & a_{01}=2.40483 \\
J_{1}\left(a_{01}\right)=0.51915 & \\
J_{0}\left(a_{11}\right)=-0.427276 & a_{11}=3.83171, \\
J_{1}\left(a_{11}\right)=0 &
\end{array}
$$

represent first zeros of the corresponding Bessel functions. The vertical center of the plasma is defined at $\mathrm{L} / 2$ in this formulation. From the force-free theory, the current densities are simply given by

$$
\vec{j}(r, z)=\overrightarrow{k B}(r, z) / \mu_{0},
$$

where $\mu_{0}=4 \pi(10)^{-7} \mathrm{H} / \mathrm{m}$ is the permeability of free space. The total toroidal, $I_{\phi}$, and poloidal, $I_{\theta}$, plasma currents are derived by integrating the appropriate current density over the cross section.

$$
\begin{aligned}
& I_{\phi}=\left(2 k^{2} / \mu_{0} k_{r}^{2} k_{z}\right)\left[1-J_{0}\left(a_{11}\right)\right] B_{0} \\
& I_{\theta}=\left(2 \pi a_{01} k / \mu_{0} k_{r}^{2}\right) J_{1}\left(a_{01}\right) B_{0} .
\end{aligned}
$$

Several magnetic field flux quantities are also of interest and are derived from the magnetic-field profiles. The total poloidal, $\psi$, and toroidal, $\phi$, magnetic fluxes are 


$$
\begin{aligned}
& \psi=\left(2 \pi a_{01} / k_{r}^{2}\right) J_{1}\left(a_{01}\right) B_{0} \\
& \phi=\left(2 k / k_{r}^{2} k_{z}\right)\left[1-J_{0}\left(a_{11}\right)\right] B_{0},
\end{aligned}
$$

while the normalized poloidal-flux function, $\Psi_{\mathbb{N}}$, which is often used in volumetrically weighted integrations, is given by

$$
\psi_{N}(r, z)=\frac{r J_{1}\left(k_{r} r\right) \sin \left(k_{z} z\right)}{\left[\left(a_{01} / a_{11}\right) R J_{1}\left(a_{01}\right)\right]},
$$

with the peak occurring at $\left(a_{01} / a_{11}\right) R=0.628 R$, as is shown in Fig. 3.1.-3. Finally, the total magnetic helicity is derived from Eq. (3.1.-1) as follows:

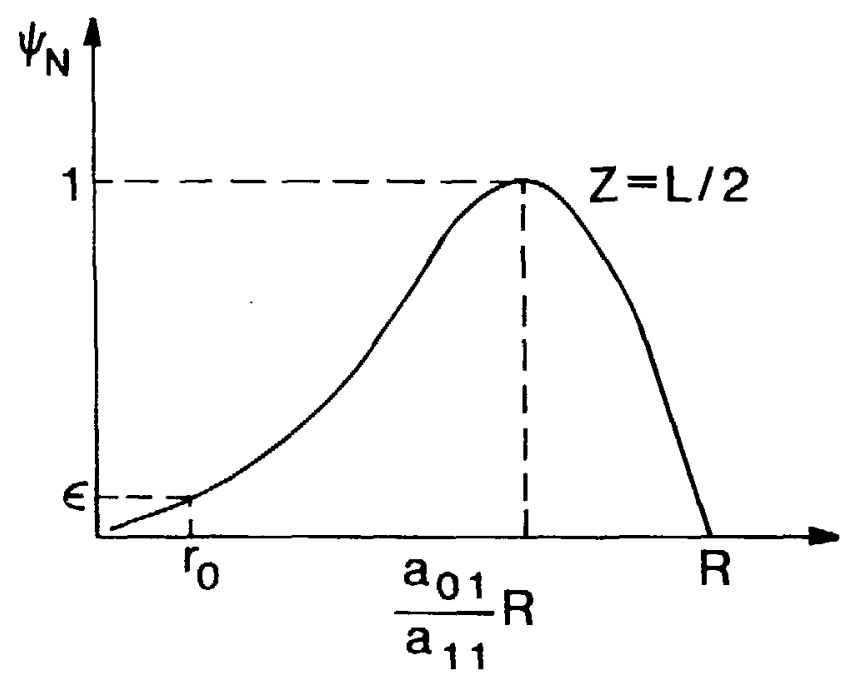

Fig. 3.1.-3. Normalized poloidal magnetic flux. 


$$
\begin{aligned}
K & =\frac{\pi a_{11}^{2} J_{0}^{2}\left(a_{11}\right)}{{ }^{4 a_{01} J_{1}\left(a_{01}\right)\left[1-J_{0}\left(a_{11}\right)\right]}} \phi \psi \\
& \simeq 1.07 \phi \psi .
\end{aligned}
$$

The value of the peak on-axis $f_{i e l d} B_{0}$, is determined by relating the average plasma pressure, $p$, needed to achieve a specific power output (Sec. 3.2.), by means of an input beta value, tr. the internal magnetic plasma energy, WINT'

$$
\mathrm{V}_{\mathrm{INT}}=\mathrm{pV}_{\mathrm{p}} / \beta
$$

where $V_{p}=\pi R^{2} L$ is the plasma volume. The input beta, $\beta$, simply relates plasma versus magnetic energy (integral of $\bmod -B^{2}$ ), and the value of $B_{0}$ is then derived from the following relationship:

$$
W_{\text {INT }}=\left(1 / 2 \mu_{0}\right) \int_{V_{p}}\left(\bmod -B^{2}\right) d V_{p}
$$

In summary, then, the foregoing expressions for field, current, and (assumed) pressure profiles are used to relate the spheromak equilibrium to the plasma power flow, gun-electrode characteristics, and overall reactor performance.

\subsection{Plasma Model}

The relationships used to calculate all plasma powers and the energy confinement time required for ignition are described in this section. The plasma output power allows the specification of the required plasma density and corresponding pressure, which is used to specify the magnetic characteristics described in Sec. 3.1. Ideally, the plasma is expected to operate near a steady-state power balance. Equating the sum of the ohmic power, $P_{O H M}$, and the alpha-particle power, $\mathrm{P}_{\alpha}$, dissipated in the plasma, to Bremsstrahlung, $\mathrm{P}_{\mathrm{BR}}$, and conduction, $\mathrm{P}_{\mathrm{COND}}$, powers gives the following steady-state power balance, 


$$
\begin{aligned}
& \mathrm{P}_{\mathrm{OHM}}+\mathrm{P}_{\alpha}=\mathrm{P}_{\mathrm{BR}}+\mathrm{P}_{\mathrm{COND}} \\
& \mathrm{g}_{\mathrm{OHM}} \eta_{\mathrm{H}}\left(\mathrm{I}_{\phi} / \mathrm{RL}\right)^{2}+\mathrm{g}_{\mathrm{DT}} \mathrm{f}_{\mathrm{D}} \mathrm{f}_{\mathrm{T}^{\mathrm{n}}}^{2}\langle\sigma \mathrm{v}\rangle \mathrm{E}_{\alpha}= \\
& g_{B R} 5.35(10)^{-37} n_{e}^{2} Z_{e f f} T^{1 / 2}+1.5\left(n_{i}+n_{e}\right) k_{B}{ }^{T^{1 /} / \tau_{E}} \quad,
\end{aligned}
$$

where $k_{B}=1.602(10)^{-16} \mathrm{~J} / \mathrm{keV}$. The average-plasma density and density-weighted average temperature are used to evaluate powers, and profile effects are included tinrough the respective profile factors, $g_{i}(i=0 H M, B R, D T)$. The following normal definitions are used: $E_{\alpha}=5.64(10)^{-13} \mathrm{~J} /$ particle, classical plasma resistivity, $n_{\|}(\Omega m)$, and $z_{\text {eff }}=\sum n_{i} z_{i}^{2} / n_{e}$. During a steady-state plasma burn, the total ion fraction consists of deuterium, $f_{D}$, tritium, $f_{T}$ and alpha particles, $f_{\alpha}$, ignoring other plasma impurities; hence, $f_{D}+f_{T}+f_{\alpha}=1$ with $n_{e}=n_{i}\left(1+E_{\alpha}\right)$. Assuming equal electron and ion temperatures, Eq. (3.2.-1) can be recast in the following form:

$n \tau_{E}=\frac{1.5\left(2+f_{\alpha}\right) k_{B} T}{g_{D T} f_{D} f_{T}\langle\sigma v\rangle E_{\alpha}-g_{B R} 5.35(10)^{-37}\left(1+f_{\alpha}\right)\left(1+3 f_{\alpha}\right) T^{1 / 2}+g_{O H M} \eta_{\| I}\left(I_{\phi} / N\right)^{2}},(3.2 .-2)$

which can be solved $f(r) n \tau_{E}$ at specific values of temperature and $I_{\phi} / N$, where the line density is given by $\mathrm{N}=\mathrm{nA}_{\mathrm{p}}$ and the plasma cross section is $\mathrm{A}_{\mathrm{p}} \equiv \mathrm{RL}$. Typically, the ohmic-heating term is small compared to the alpha-particle power, as is required for a high-Q plasma system. The value for $n \tau_{E}$ is then derived from Eq. (3.2.-2) assuming the ohmic heating term is small and all ions are lost at the same rate. The iterative solution of this equation in this case only needs to be obtained as the plasma temperature or profile functions are changed. once the plasma ion density, $\pi_{i}$, is known, the required energy confinement time, $\tau_{E}$, can then be determined by Eq. (3.2.-2). The average plasma density is given by

$$
n_{i}=\left[P_{\alpha} /\left(f_{D} f_{T} g_{D T}\left\langle\sigma v>E_{\alpha} V_{p}\right)\right]^{1 / 2},\right.
$$

where the alpha-particle power, $P_{\alpha}$, is dictated by the required reactor thermal power. The plasma pressure is defined as follows: 


$$
P \equiv n_{i}\left(2+f_{\alpha}\right) k_{B} T,
$$

which specifies all the plasma magnetic properties using the relationships given in Sec. 3.1 .

All the plasma powers can be evaluated once the profile weighting functions are known. A typical plasma parameter, $P(r, z)$, can be represented as a function of the plasma position $(r, z)$ by

$$
P(r, z)=f[B(r, z), j(r, z), n(r, z), T(r, z)],
$$

the toroidal plasma current, $I_{\phi}$, a volumetric averaged density, $n$, and a volume-averaged density-weighted temperature, $T$. The above definitions are arbitrary, with specific definitions producing a specific set of weighting functions. The weighting functions, $g_{i}$, are then calculated from the two-dimensional integral

$$
g_{i}=\frac{2 \pi \int V_{p} P(r, z) r d r d z}{P V_{p}} .
$$

The above formulation gives the peak-to-average values for density, $n_{0} / n$, and density-weighted temperature, $T_{0} / T$, with the function $P(r, z)$ characterized by the magnetic field, $B(r, z)$, current density, $j(r, z)$, plasma density, $n(r, z)$ and temperature, $T(r, z)$. These parameters are reduced to profile-averaged quantities given by

$$
P=g_{i} f\left(B, j_{\phi}, n, T\right),
$$

where the normalizing parameters for magnetic field, current density, particle density, and temperature are given as follows: 


$$
\begin{aligned}
B & \equiv B_{0} \\
j_{\phi} & \equiv I_{\phi} / A_{p} \\
n & \equiv \int_{V_{p}} n(r, z) 2 \pi r d r d z / V_{p} \\
T & \equiv \int_{V_{p}} T(r, z) n(r, z) 2 \pi r d r d z / V_{p} n .
\end{aligned}
$$

This formulation uses the peak field, $B_{O}$, and the profile weighting functions for fusion, $g_{D T}$, Bremsstrahlung, $g_{B R}$, and ohmic-heating, $g_{O H M}$, powers.

Since the magnetic-field profiles are defined in Eq. (3.1.-2) and the current densities are defined by Eq. (3.1.-5), only the plasma density and temperature need be specified to calculate all weighting functions. The density and temperature profiles are taken to be proportional to the plasma flux

$$
n(r, z) \propto T(r, z) \propto \psi_{N}(r, z)
$$

which is defined by Eq. (3.1.-8).

\subsection{Current-Drive (Electrode/Gun) System}

With the plasma magnetics (Sec. 3.1.) and all plasma powers (Sec. 3.2.) defined, the determination of the electrical input to the plasma required to sustain the plasma currents can be made. The magnitude of ohmic power loss was defined in Sec. 3.2. to be

$$
P_{\mathrm{OHM}}=g_{\mathrm{OHM}} \eta_{\mathrm{II}}\left(I_{\phi} / \mathrm{RL}\right)^{2} \mathrm{~V}_{\mathrm{p}}
$$

again using classical resistivity evaluated at the density-weighted average temperature, $T$, with $\mathrm{g}_{\mathrm{OHM}}$ accounting for all other current and profile effects. This ohmic dissipation can be expressed in terms of a magnetic-fiel」 energy decay time, $\tau_{B^{2}}$, as follows:

$$
\tau_{\mathrm{B}^{2}}=\mathrm{W}_{\mathrm{INT}} / \mathrm{P}_{\mathrm{OHM}},
$$


where $W_{\text {INT }}$ is the total magnetic energy stored in the plasma using Eq. (3.1.-11) for the internal plasma energy. This relaxation time also dictates the required refluxing rate or rate of helicity injection, which is given by

$$
\mathrm{dK} / \mathrm{dt}=\mathrm{K} / \tau_{\mathrm{B}^{2}},
$$

and must be provided by the plasma gun during the plasma burn.

The geometry used to analyze the gun properties is illustrated in Fig. 3.3.-1. A fraction, $\varepsilon$, of the poloidal flux, $\psi$, is diverted to the gun, where the expression

$$
\varepsilon=\frac{\left.r_{0} J_{1} ; k_{r_{0}} r_{0}\right)}{\left(a_{01} / a_{11} ; R J_{1}\left(a_{01}\right)\right.}
$$
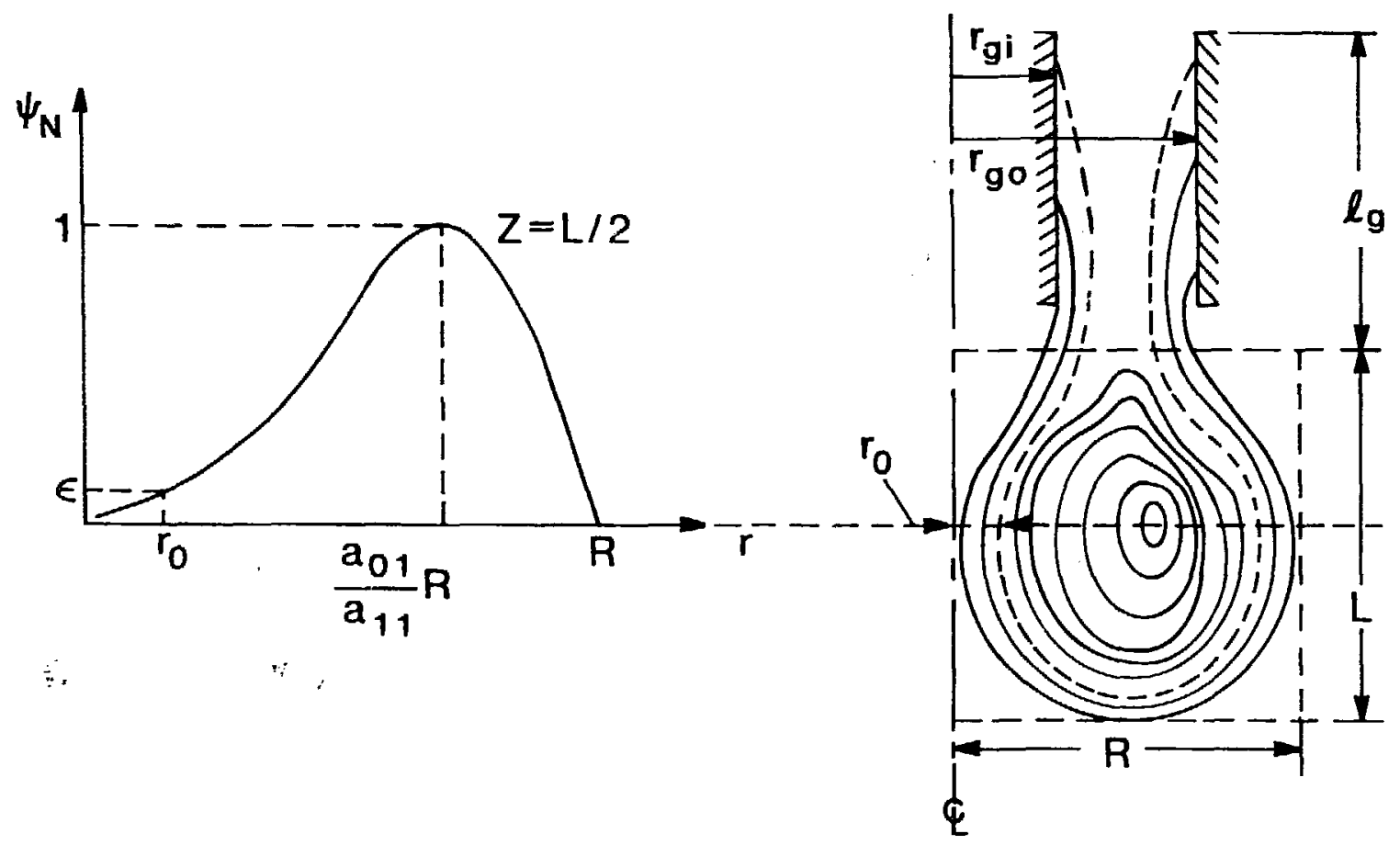

Fig. 3.3.-1. Geometry used to analyze the gun properties where the fraction of flux, $\varepsilon$, is diverted inside the radius, $r_{0}$, at the plasma center. 
is derived from the normalized flux $\psi_{\mathrm{N}}$ and $r_{0}$ is an on-axis radius of the flux tube diverted to the gun (Fig. 3.3.-1). The total helicity injection rate of the plasma gun ${ }^{68}$ is given by

$$
\mathrm{dK} / \mathrm{dt}=2 \varepsilon \psi \mathrm{V}_{\mathrm{gK}},
$$

which can be combined with Eq. (3.3.-3) to estimate the gun voltage, $V_{\text {gK, needed }}$ for helicity injection according to the following expression:

$$
\mathrm{V}_{\mathrm{gK}}=\left(\mathrm{K} / \tau_{\mathrm{B}^{2}}\right) /(2 \varepsilon \psi)
$$

The current density that must be driven on this diverted flux surface must as a minimum equal the Taylor current $\left(j \simeq \mathrm{kB}_{z} / \mu_{0}\right.$ evaluated at $\left.r_{0}\right)$, as suggested by the model of relaxed states discussed in Sec. 3.1. If magnetic helicity could be injected at this current density, the efficiency would optimistically be 100\%. Accounting for somewhat lower efficiencies gives a gun/electrode or divertor current of

$$
I_{g}=\pi r_{o}^{2} j=\pi r_{o}^{2}\left(k B_{z} / \mu_{o}\right) / \varepsilon_{K}
$$

where $\varepsilon_{K}$ is the injection efficiency typically taken to be $\sim 1 / 1.5$ (i.e., requiring a current density $\sim 50 \%$ greater than the Taylor current). These somewhat higher current densities are experimentally shown to be absorbed into the plasma volume, effectively refluxing the plasmoid; the actual efficiency of this process is estimated to be $-25 \%$.

Driving flux from the gun/electrode to the spheromak is also expected $t$ be accompanied by parasitic losses, including an ohmic voltage drop, $V_{g R}$, in "hie diverted flux channel. In addition, an arc voltage drop, $\mathrm{V}_{\mathrm{gA}}$, across he electrode/gun surface is expected and is a quantity that must be experimentaliy derived. Both resistive losses in the divertor channel and the arc drop are estimated and included in the CSR analyses. 
Derivation of the resistive voltage drop across the divertor channel requires specification of the plasma/gun geometry. Considering the asymmetry associated with the inside, $r_{g i}$, and outside, $r_{g o}$, radii of the gun and the diverted flux which ties both electrode surfaces together, such an estimate is not easily made. The outside radius of the gun is defined from the following relationships 58

$$
\begin{aligned}
& r_{g o} \leq\left(k_{r} / k\right) R \\
& r_{g i} \simeq(2 / 3) r_{g o},
\end{aligned}
$$

where in performing this study the equalities are presumed in both cases. The specification of $r_{g o}$ is derived from the requirement that the magnetic energy per unit helicity in the spheromak be less than or equal to the same quantity in the gun system, using the Taylor relaxation mechanism to force flux out of the gun into the spheromak plasma. The expression for inside radius, $r_{g i}$, is based upon the coaxial aspect ratio used in experiments.58 This electrode surface for the reactor must be capable of extracting the charged-particle and thermal-conduction power streaming from the spheromak plasma. Invoking a maximum heat loading at the gun/electrode surface, $\mathrm{I}_{\mathrm{OW}}\left(\mathrm{MW} / \mathrm{m}^{2}\right)$, the total length of the gun/electrode, based upon thermal energy extraction, is then given by

$$
{ }_{g} \geq P_{q}(H) /\left[I_{Q w} 2 \pi\left(r_{g o}+r_{g i}\right)\right]
$$

where $P_{q}(W)$ is the total thermal power to the electrodes if uniformly distributed. The average divertor channel half-length is defined as

$$
Q / 2=L+R+\ell_{g} / 2,
$$

and an area variation along this channel length, $2 / 2$, of the following form is assumed:

$$
A(x)=A_{n}[\rho+x(1-p)]_{x=0 \text { (plasma) }}^{x=l / 2 \text { (gun) }}
$$




$$
\begin{aligned}
& A_{n}=\pi l_{g}\left(r_{g o}++r_{g i}\right) \\
& A_{0}=\pi r_{0}^{2} \\
& \rho=A_{0} / A_{R},
\end{aligned}
$$

where a linear area variation with length is assumed. Using this characterization of the channel permits an analytic solution to the heat conduction equation

$$
P_{q}(w)=-k(T) A(x)(d T / d x),
$$

where the parallel heat conductivity is given by

$$
k(W / k e V m)=9.8(10)^{14} T^{5 / 2} / \ln \Lambda .
$$

Assuming all the heat enters at $x=0$ and internal heating does not significantly impact the temperature profile, the temperature distribution along the channel is estimated to be given by the following expression:

$$
T(x)=\left\{\frac{7}{2} \frac{l n \Lambda}{9.8: 10)^{14}} \frac{P_{q}}{A_{n}} \frac{l / 2}{1-\rho} \ln \left[\frac{1}{\rho+\frac{x}{l / 2}(1-\rho)}\right]+T_{d}^{7 / 2}\right\}^{2 / 7},
$$

where $T_{d}$ is the surface electrode boundary condition and is typically taken to be $3 \mathrm{eV}$. The temperature at the plasma surface may be found by setting $\mathbf{x}=0$ in Eq. (3.3.-14). The temperature distribution can then be evaluated upon specifying the power flowing in each channel, $P_{q}(W)$, which is typically taken as the plasma conduction power (approximately the alpha-particle power, $\mathrm{P}_{\alpha}$ ) divided according to the available surface area of each respective electrode. As indicated by Eq. (3.3.-14), the temperature distribution is a weak function of the specific area variation along the channel and also the power assumed to exit through each channel. This observation that the resistive power loss, as found 
by a numerical integration along the channel length (Sec. 3.4.), is relatively small when compared to the thermal conduction power from the plasma to the electrode justifies use of the simplified model. In summary, the assumptions of no heat addition or loss along the channel, the channel area varying linearly with length, and the power splitting along the channel according to the electrode surface area, are not expected to introduce unacceptable errors to the reactor systems analysis. This issue is examined in more detail in Appendix A, particularly with respect to the "watershed" point for heat flow out of the plasma and the division of this heat between inner and outer electrodes.

Using the resistive power loss in the channel ( $\mathrm{Sec} .3 .4$. ), $\mathrm{P}_{R}$, to give the channel resistive voltage drop according to $V_{g R}=P_{R} / I_{g}$, the total voltage impressed on the gun, $V_{g}$, is given by the sum of helicity injection, resistive, and arc voltages, respectively.

$$
V_{g}=V_{g K}+V_{g R}+V_{g A}
$$

The total voltage on the gun is then specified if the required divertor channel current, $I_{g}$, can be identified. As suggested by Eq. (3.3.-4) and Eq. (3.3.-7), a definition of the poloidal flux fraction, $\varepsilon$, diverted to the electrode surface is required. The value of $r_{0}$ and $\varepsilon$ can be uniquely defined when the plasma gun operating characteristics are considered. A plasma/gun scaling suggested by experiment can be derived from Fig. 3.3.-2 and is given by

$$
I_{g} / V_{g}^{1 / 3}=8(10)^{3}[1.0+110 \varepsilon \psi]
$$

Although the $V_{g} \propto I_{g}^{3}$ dependence can be predicted on the basis of mass-flow and magnetic-flux balances at the gun electrode, the flux dependence shown in Eq. (3.3.-16) is not understood. It is likely that the coefficient multiplying the $\varepsilon \psi$ term in Eq. (3.3.-16) may exhibit a dependence on gun geometry; ideally, such a coefficient would decrease as the gun size is increased. For the purposes of this study and until an experimental geometry scaling becomes available, the $100 \varepsilon \psi$ term in Eq. (3.3.-16) will be paramet._cally reduced by a factor $f_{R}$. As is shown in Sec. 4.1, $f_{R}=1$ will disallow systems of sufficient physical size to include the minimum-COE designs, whereas $f_{R} \leq 0.5$ allows all 


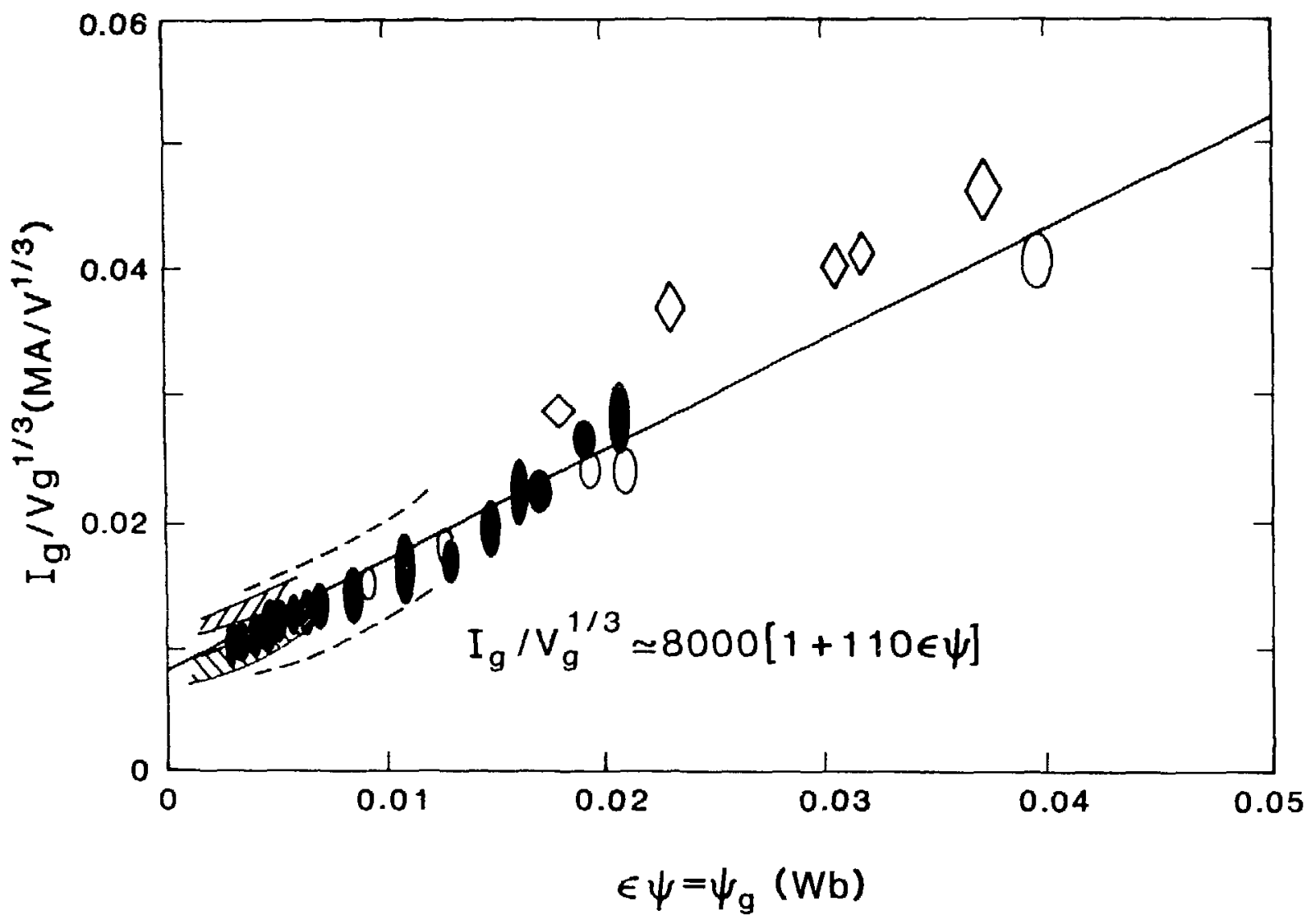

Fig. 3.3.-2. Plasma-gun "law" relating the major parameters $V_{g}, I_{g}$, and $\varepsilon \psi$.

designs of economic interest; the specific value of $f_{R}$ forms a design cutof $f$ rather than impacting the shape and slope of specific design optima. Generally, Eq. (3.3.-16) is consistent with the required helicity injection and divertor resistive loss at unique values of $\varepsilon$ and $r_{0}$, which must be determined by iterative solution, as is discussed in Sec. 2.2. The fraction of poloidal flux that typically is required to be diverted from the plasma is $\varepsilon \leq 0.05$.

The plasma and gun/electrode parameters used for the basecase (optimal) systems studies solution are listed in Table 3.3.-I. The magnetic-field model is discussed in Sec. 3.1., with near-optimal equilibrium/stability characteristics 67 being predicted for $L / R \simeq 1.25$. The plasma profile and beta information are experimentally inferred,58,84,85 while a plasma operating temperature of $20 \mathrm{keV}$ is shown in Sec. 4. to be nearly optimal. The 


\section{PHYSICS INPUT BASECASE VALUES}

\section{PLASMA}

Magnetic field model

Aspect ratio, L/R

Pressure profile

Density, temperature profile

Fuel

Beta, $\beta$

Average temperature, $\mathrm{T}(\mathrm{keV})$

Edge-plasma temperature, $\mathrm{T}(\mathrm{keV})$

Impurity content, $\mathrm{Z}_{\text {eff }}$

\section{DIVERTED FLUX SURFACE}

Temperature profile

Divertor current, $1 / \varepsilon_{\mathrm{K}}=\mathrm{I}_{\mathrm{g}} /($ Taylor current)

ELECTRODE/GUN

Gun electrode radius ratio, $r_{g i} / r_{g o}$

Helicity injection efficiency, $\varepsilon_{\mathrm{K}}$

Electrode surface temperature, $\mathrm{T}_{\mathrm{D}}^{\mathrm{k}}(\mathrm{keV})$

Plasma gun scaling

Electrode arc voltage, $V_{g A}(V)$ $\mu=\mu_{0} \frac{\text { VALUE }}{\vec{j} \cdot \vec{B} / B^{2}}=$ CONSTANT

1.25

$\psi^{2}$

$\Psi$

DT

0.1

20.0

0.5

1.0

Eq. $(3 \cdot 3 \cdot-14)$

divertor-channel and helicity-injection parameters are consistent with the expected behavior discussed in this section.

\subsection{Engineering Power Balance}

The steady-state CSR energy balance used in this study is shown in

Fig. 3.4.-1. The plasma power production includes neutron, $\mathrm{P}_{N}=4 \mathrm{P}_{\alpha}$, radiation, $\mathrm{P}_{\text {RAD }}$, and conduction, ${ }^{\mathrm{P}}$ COND, powers, as described by the appropriate terms in Eq- (3.2.-1). The current-drive or helicity-injection power, $P_{K}$, is delivered to the plasma with an efficiency $\varepsilon_{K}$; the balance of this power, $\left(1-\varepsilon_{K}\right) P_{K}$, is assumed to be delivered to the thermal-conversion system. This plasma refluxing is accompanied by two other parasitic losses, as discussed in Sec. 3.3. Flux. injection from the gun/electrode requires current to be driven along the flux lines that connect the gun to the plasma, leading to a resistive power loss, $P_{R}$. In addition, an arc drop by the electrode surface will induce an additional power loss, $\mathrm{P}_{\mathrm{A}}$. The total power to the gun is then simply the sum of these three power flows (i.e., $P_{g}=P_{K}+P_{A}+P_{R}$ ). Assuming all of the gun power, $P_{g}$, 


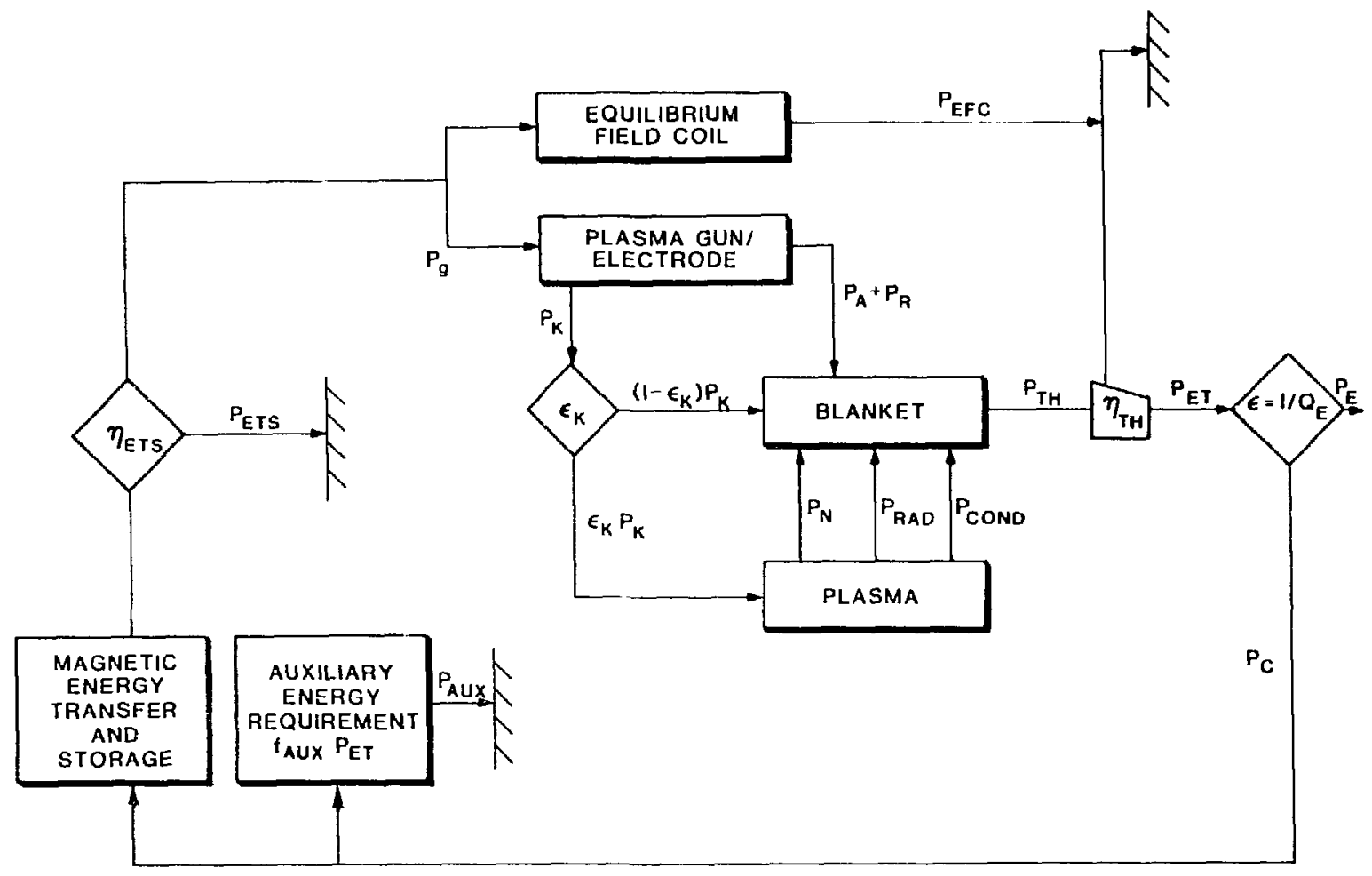

Fig. 3.4.-1. Steady-state energy balance used in the CSR systems model.

is thermally recoverable, multiplying the neutron power by the blanket multiplication, $M_{N}$, ignoring ohmic heating in the spheromak per se, and adding the alpha-particle power, $\mathrm{P}_{\alpha}=\mathrm{P}_{\mathrm{RAD}}+\mathrm{P}_{\mathrm{COND}}$, gives the total thermal power, $\mathrm{P}_{\mathrm{TH}}$, delivered to a conventional steam turbine, which in turn operates with a thermal efficiency, $\eta_{\mathrm{TH}}$. A fraction, $\varepsilon \equiv \mathrm{i} / \mathrm{Q}_{\mathrm{E}}$, of the resulting gross electric power, ${ }^{P_{E T}}=\eta_{T H} P_{T H}$ is used to prcvide recirculating power, ${ }_{C}$, to drive the reactor system, leaving a net electric power, $\mathrm{P}_{E}=\mathrm{P}_{\mathrm{ET}}(1-\varepsilon)$ deliverable to the grid. The net plant efficiency, therefore, is $\eta_{\mathrm{p}}=(1-\varepsilon) \eta_{\mathrm{TH}}$. The recirculated power supplies plant auxiliary loads, $\mathrm{P}_{\mathrm{AUX}}$, and the power required by the coil/plasma system. Ohmic dissipation in the resistive equilibrium-field coils, $\mathrm{P}_{\mathrm{EFC}}$, and the plasma current-drive system, $P_{g}$, are driven with an efficiency $\eta_{E T S}$ and correspondingly require electrical loss, $P_{E T S}$, to be supplied. The recirculating power fraction is given by 


$$
Q_{E}=1 / \varepsilon=P_{E T} / P_{C}=\eta_{T H} \frac{M_{N} P_{N}+P_{R A D}+P_{C O N D}+P_{g}}{P_{g}+P_{E F C}+P_{A U X}+P_{E T S}},
$$

where $Q_{E}=1 / \varepsilon$ is defined as the engineering $Q$-value.

Engineering parameters used to evaluate the reactor energy balance are listed in Table 3.4.-I. For this steady-state system, the loss associated with delivering (startup) power to the magnets is considered small (i.e., P $_{\text {ETS }} \simeq 0$ ). The auxiliary plant requirements, including all other plant loads, are taken as a fraction $\mathrm{f}_{\mathrm{AUX}}=0.07$ of the gross electric power, $\mathrm{P}_{E T}$. Only the ohmic loss for the normal conducting coil, $P_{E F C}$, and refluxing requirements, $P_{g}$, affects directly the FPC and remains to be evaluated in terms of specific reactor characteristics.

Enforcing plasma equilibrium requires a nearly uniform vertical field produced by an EFC of radius, $r_{C}=r_{H}+\Delta b+\Delta r_{C} / 2$, as shown in Fig. 2.2.-1. Driving a uniform current in this coil configuration produces a vertical field with a negative magnetic field index in the range $-1.5<\left(r / B_{V}\right) \partial B_{V} / \partial r<0$, as is shown in Fig. 3.4.-2, and is necessary to provide equilibrium in the vertical

TABLE 3.4.-I

\section{ENGINEERING INPUT (BASE/OPTIMAL CASE)}

Fusion Power Core

Blanket thickness, $\Delta b(m)$ $\frac{\text { Value }}{0.6}$

Neutron blanket multiplication, $\mathrm{M}_{\mathrm{N}}$ 1.3

Normal/superconducting coil Normal

Coil resistivity, $n_{\mathrm{Cu}}\left(\Omega_{\mathrm{m}}\right)$ $1.8(10)^{-8}$

Coil-conductor filling fraction, $\lambda_{\mathrm{c}}$ 0.7

Electrode radius ratio, $\mathrm{r}_{\mathrm{gi}} / \mathrm{r}_{\mathrm{go}}$

Design electrode heat load, $\mathrm{I}_{\mathrm{QW}}\left(\mathrm{MW} / \mathrm{m}^{2}\right)$

Number of current hoops to model plasma/EFC, $N_{p} / N_{E F}$ $80 / 12$

Balance of Plant

Thermal conversion efficiency, $\eta_{T H}$

Auxiliary power fraction, $f_{A U X}$

Energy transfer efficiency, $n_{\text {ETS }}$

1.0 

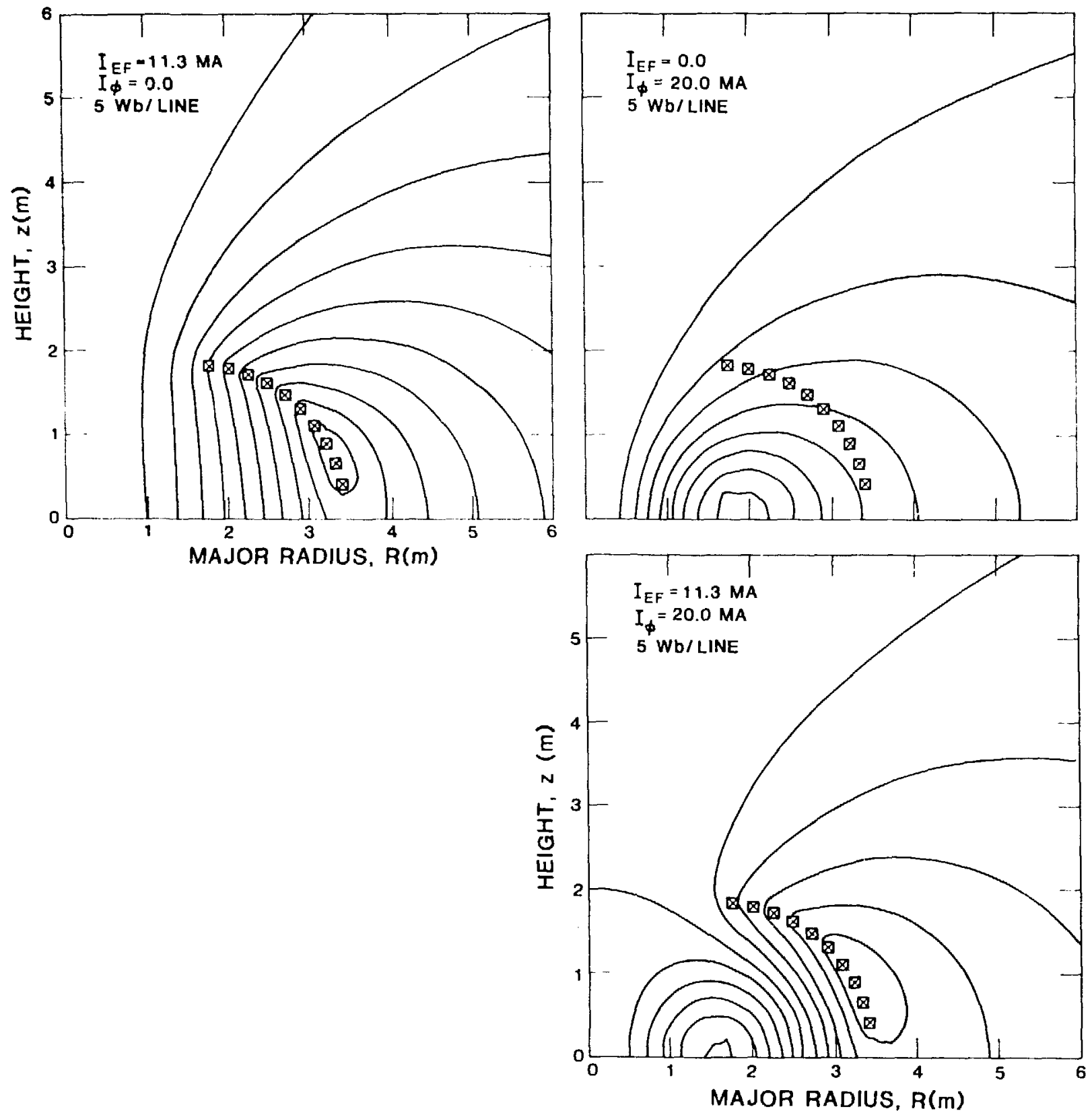

Fig. 3.4.-2. Equilibrium-field coil, plasma, and combined poloidal fluxes for CSR basecase design. 
direction.87-89 Calculating the equilibrium field current, $I_{E F}$, flowing in this EFC configuration requires a numerical solution. For a given EFC current, the flux produced is calculated by representing this conductor set as a number of circular hoops from which the magnetic properties are found from elliptic integrals.90 From the desired plasma equilibrium (Sec. 3.1.), the plasina toroidal current, $I_{\phi}$, can also be represented by a set of circular hoops calculated exactly from the following expression:

$$
\begin{aligned}
I_{\phi}(\Delta z, \Delta r)= & \frac{B_{0}}{\mu_{0}} \frac{k^{2}}{k_{z} k_{r}^{2}}\left\{\cos \left(k_{z} z\right)-\cos \left[k_{z}(z+\Delta z)\right]\right\} \\
& \left\{J_{0}\left(k_{r} r\right)-J_{0}\left[k_{r}(r+\Delta r)\right]\right\} .
\end{aligned}
$$

The total toroidal current is given in Eq. (3.1.-6), where all parameters have been previously defined (Sec. 3.1.). The flux profile generated by the toroidal plasma current is then calculated analogously to the EFC flux profile determination. Since the plasma equilibrium presumed a close-fitting, highly conducting shell, the magnetic flux produced only by the internal toroidal plasma current will generate a separatrix (or plasma) radius essentially at infinity along the major toroidal axis, R, as is illustrated in Fig. 3.4.-2. The total EFC current is determined by requiring the poloidal flux, $\psi(R, L / 2)$, to be zero, as dictated by the assumed analytic equilibrium. This procedure simply positions the plasma separatrix at the proper design location, yielding a specific value for the current ratio $I_{E F} / I_{\phi}$ without requiring the absolute magnitude of either. The plasma and EFC are typically represented by $N_{p}=80$ and $N_{E F}=12$ loops, respectively, as is noted in Table 3.4.-I. With the plasma and EFC currents determined, the calculation of ohmic losses in the EFC can be made. Using the equivalent loop conductor positions also permits the calculation of the plasma self-inductance, $\mathrm{L}_{\mathrm{p}}$, EFC inductance, $\mathrm{L}_{E F}$, and mutual inductance, ${ }^{\mathrm{H}} \mathrm{EF}, \mathrm{p}$, using the appropriate flux/current linkage formulation. The total stored energy in the plasma/EFC system, $W_{B}$, is then given by

$$
W_{B}=\frac{1}{2} L_{P} I_{\phi}^{2}-M_{E F, p} I_{\phi} I_{E F}+\frac{1}{2} L_{E F} I_{E F}^{2}
$$


Finally, the power input requirement to the plasma gun, $\mathrm{P}_{\mathrm{g}}$, must be determined. The current-drive requirement is related to the required helicity injection rate, $\mathrm{dK} / \mathrm{dt}$, given in Sec. 3.4., which required the gun voltage, $V_{g K}$ ' and corresponding injected power, the latter being given by

$$
\mathrm{P}_{\mathrm{gK}}=\mathrm{V}_{\mathrm{gK}} \mathrm{I}_{\mathrm{g}}=\mathrm{P}_{\mathrm{OHM}} / \varepsilon_{\mathrm{K}}
$$

where $I_{g}$ is the divertor channel current given by Eq. (3.3.-7).

The total power dissipated in the divertor channel, $P_{R}$, is found by integrating the local ohmic dissipation, $n_{\|} j_{\phi}^{2}$, along the channel using the following expression:

$$
P_{g R}=2 \int_{0}^{\ell / 2} n_{I I}(T)\left[\frac{j\left(r_{0}\right) \rho}{\rho+f(x)[1-\rho]}\right]^{2} A(x) d x,
$$

where the area functions, $p$, along the divertor channel length have been defined by Eq. (3.3.-11). In this formulation both inside and outside channels are assumed identical, with the integral along the inside path multiplied by two. This approximate formulation is considered adequate because of the typically small resistive power dissipated in the divertor channel. This issue is explored further in Appendix A. The current density given by Eq. (3.3.-7) at $r_{0}$ is adjusted along the divertor channel for area changes. The classical plasma resistivity is evaluated along the temperature profile specified by Eq. (3.3.-14).

Finally, an arc voltage drop, $v_{g A}$, may be required at the electrode to sustain the divertor channel current. The power dissipated by the arc voltage is given by

$$
\mathrm{P}_{\mathrm{gA}}=\mathrm{V}_{\mathrm{gA}} \mathrm{I}_{\mathrm{g}}
$$

All power loss channels associated with the plasma-gun/electrode system are thereby specified. 


\subsection{Economics}

Economic guidelines recommended $4,20,21$ to assure uniformity of comparisons among fusion reactor studies serve as the basis for the costing framework used in the CSR study. The costing guidelines describe uniform accounting categories and procedures, as summarized in Table 3.5.-I, which also gives details of the cost database. As shown in Fig. 3.1.-1, the total cost of the plant is comprised of direct, indirect, and time-related (EDC and IDC) costs. Direct costs are quoted throughout this study on the basis of 1980 prices and result from the purchase of materials, equipment, and labor. These costs include allowances for spare parts and contingencies added as a percentage to the subtotals of Accounts 20-26 (Table 3.5.-I). Indirect costs are taken as a percentage of the direct costs and result from support activities necessary to complete the project. The indirect costs are divided into three major accounts: $10 \%$ for construction facilities, equipment and services; $8 \%$ for engineering and construction management services; and $5 \%$ for taxes, insurance, staff training, and plant startup. Escalation and interest are computed as a percentage of the base (direct plus indirect) costs, assuming a 5-year construction period. Aggregate percentages of $25.1 \%$ and $15.5 \%$, respectively, result for an interest rate of $10 \% / y r$ and escalation rate of $5 \% / y r$. Having computed the unit total cost, UTC( $\$ / k W e)$, the cost of electricity, $\operatorname{COE}(\mathrm{mills} / \mathrm{kWeh})$, can then be computed. The assumptions used in calculating the COE are listed in Table 3.5.-II and incorporated into the following relationship.

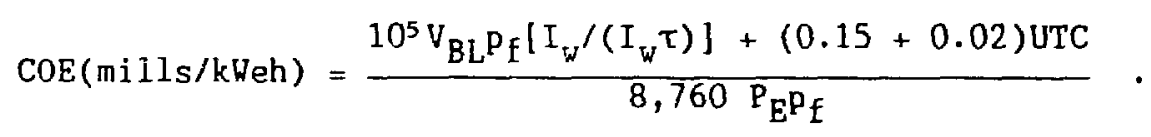

The yearly replacement cost associated with the blanket volume, $V_{B L}, 15 \%$ return on investment, and $2 \%$ operating costs are divided by $8,760 \mathrm{~h} / \mathrm{y}$, the net electric power, $P_{E}$ (MWe), and the plant factor, $P_{f}$, to give the COE. Table 3.5.-II also lists the procedure by which $p_{f}$ is computed, with the first-wall/blanket lifetime, $I_{w} \tau\left(M V y r / m^{2}\right)$, being an input (and varied) parameter. The economic penalty for high neutron wall loadings, $I_{w}\left(\mathrm{MW} / \mathrm{m}^{2}\right)$ is thereby reflected through the reduced plant factor. The unscheduled time the plant is considered off 1 ine is taken from the STARFIRE tokamak reactor study. ${ }^{4}$ 
TABLE 3.5.-I

SUMMARY FUSION REACTOR COST DATABASE ${ }^{(a)}$

ACC. NO

ACCOUNT TITLE

20. Land and land rights

(M\$, 1980)

3.3

21.

21.1

21.2

21.3

21.4

21.5

21.6

21.7

21.98

21.99

22.

22.1

22.1 .1

22.1.2

Structures and site facilities

Site improvements and facilities

Reactor building

Turbine building

Cooling structures

Power supply and energy storage

Miscellaneous buildings

Ventilation stack

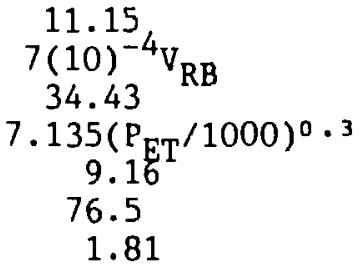

Spare parts $(2 \%)$

Contingency (15\%)

Reactor Plant Equipment

Reactor Equipmen $t$

Blanket and first wall $0.19 \mathrm{~V}_{\mathrm{BL}}$

Shield

Magnets

Supplemental heating systems

0.0

22.1 .4

Primary structure and support

Reactor vacuum system

Power supply (switching, energy storage)

Impurity control system

Direct energy conversion

$0.28 \mathrm{~V}_{\mathrm{C}}$

0.0

22.1 .6

22.1 .7

22.1 .8

22.1 .9

22.2

22.3

22.4

22.5

22.6

22.7

22.98

Primary coolant (PbLi)

$0.16 \mathrm{~V}_{\text {STR }}$

$0.0051 V_{\text {VAC }}^{R}$

$0.04 \mathrm{P}_{\mathrm{ET}}$

$0.0026^{\mathrm{ET}} \mathrm{V}_{\mathrm{VAC}}$

0.0

Auxiliary cooling

Radioactive waste treatment

Fuel handling and storage

0 ther reactor plant equipment

Instrumentation and control

22.99 Contingency allowance (15\%)

23. Turbine plant equipment

23.1

23.2

23.3

23.4

23.5

23.6

23.7

23.98

Turbine-generators

Main steam system

$0.0175 \mathrm{P}_{\mathrm{TH}}$
$6.7(10)^{-4} \mathrm{P}_{\mathrm{P}}$

$6.7(10)^{-4} \mathrm{P}_{\mathrm{TH}}$

$1.2(10)^{-3} \mathrm{P} T H$

$9.65(10)^{-3} \mathrm{P}_{\mathrm{TH}}^{\mathrm{H}}$

$0.0109 \mathrm{P}_{\mathrm{TH}}$

23.41

Heat reject: on systems

Condensing system

Feed heating system

0 ther turbine plant equipment

Instrumentation and control

23.99 Contingency allowance (15\%)

$59.9\left(\mathrm{P}_{\mathrm{ET}} / 1000\right)^{0.7}$

$4.8 \quad\left(\mathrm{P}_{\mathrm{TH}} / 2860\right)$

$33.0\left(\mathrm{P}_{\mathrm{TH}}^{\mathrm{TH}} / 2860\right)^{0.8}$

$13.8\left(\mathrm{P}_{\mathrm{ET}}^{\mathrm{TH}} / 1000\right)^{0.9}$

$7.55\left(\mathrm{P}_{\mathrm{TH}}^{\mathrm{T}} / 2860\right)$

$40.9\left(\mathrm{P}_{\mathrm{ET}} / 1000\right)^{0.6}$

$7.8\left(\mathrm{P}_{\mathrm{ET}} / 1000\right)^{0.3}$ 
TABLE 3.5.-I (cont)

SUMMARY FUSION REACTOR COST DATABASE ${ }^{(a)}$

ACC. NO

ACCOUNT TITLE

(M\$S, 1980)

24. Electric plant equipment

24.1

24.2

24.3

24.4

24.5

24.6

24.7

24.98

24.99

Swi tchgear

Station service equipment

Swi tchboards

Protective equipment

Electrical structures and wiring containers

Power and control wiring

Electrical lighting

Spare parts allowance (4\%)

Contingency allowance (15\%)

$8.6\left(\mathrm{P}_{\mathrm{ET}} / 1000\right)$

$5.4\left(\mathrm{P}_{\mathrm{ET}} / 1000\right)^{2}$

2.11

17.4

35.99

8.2

25.

25.1

25.2

25.3

25.4

25.98

Miscellaneous plant equipment

Transportation and lifting equipment

15.68

Air and water service systems

12.35

Communications equipment

6.22

Furnishings and fixtures

1.20

Spare parts allowance (3\%)

25.99 Contingency allowance (15\%)

26. Special Materials

$0.25+0.0902\left(3 \mathrm{~V}_{\mathrm{B} !}\right)$

90. Total reactor direct capital cost

91. Construction facilities, equipment, and services (10\%)

92. Engineering and construction management services (8\%)

93. other costs (5\%)

94. Interest during, construction, (25.1\%, 5 yrs e 10\%/yr)

95. Escalation during construction, (15.5\%, 5 yrs e $5 \% / y r$ )

99. Total reactor capital cost

(a) Gross electric, $P_{E T}$, net electric, $P_{E}$, and total thermal, $P_{T H}$, powers given in MW. Volumetric $\left(\mathrm{m}^{3}\right)$ abbreviations, specific masses, and equivalent mass costs in $(\$ / \mathrm{kg})$ for the fusion power core are given as follows:

Reactor building: $\mathrm{V}_{\mathrm{RB}}=10 \pi\left(\mathrm{R}_{\mathrm{T}}+5\right)^{2}+1.2(10)^{5}$

Blanket: structure $\left(\mathrm{B}_{\mathrm{BL}} \times 1000 \mathrm{~kg} / \mathrm{m}^{3} \times 100 \$ / \mathrm{kg}\right)+$ Coolant $\left(\mathrm{V}_{\mathrm{BL}} \times 9(10)^{4} \$ / \mathrm{m}^{3}\right)$

Magnet: $V_{c} \times 7300 \mathrm{~kg} / \mathrm{m}^{3} \times 38 \mathrm{~s} / \mathrm{kg}$; includes insulation and structure, copper cost is $50 \$ / \mathrm{kg}$.

Structure: $V_{\text {STR }} \times 7800 \mathrm{~kg} / \mathrm{m}^{3} \times 20 \$ / \mathrm{kg}$

Vacuum: $V_{V A C}=(4 / 3) \pi R^{3}$ 
TABLE 3.5.-II

COST-OF-ELECTRICITY (COE) MODEL USED FUR CRFPR STUDY

\section{PARAMETER}

Return on investment (\%)

operating cost (\%)

First-wall/blanket lifetime, $I_{W} \tau\left(M W y r / m^{2}\right)$

Blanket costs per replacement $(S)$

Plant factor, $p_{f}=\left(365-\tau_{u}-\tau_{S}\right) / 365$

- unscheduled, $\tau_{u}$ (days/yr)

- scheduled, $\tau_{s}$ (days/yr)

$>1$ replacement/y

$<1$ replacement/y
VALUE

15

2

15

$0.10(10)^{6} \quad \mathrm{~V}_{\mathrm{BL}}$

$\leq 0.76$

60

$28 \mathrm{p}_{\mathrm{f}} \mathrm{I}_{\mathrm{W}} /\left(\mathrm{I}_{\mathrm{W}} \tau\right)$

28

Each first-wall/blanket replacement is conservatively considered to require 28 days. Excessive downtime leading to reduced plant factor creates an impetus for avoiding very high neutron wall loadings. 


\section{RESULTS}

\subsection{Basecase Parameters}

This section gives the results of the basecase systems analysis used to determine CSR design-point sensitivities (Sec. 4.2.). The basecase presented herein corresponds to a minimum-COE design. Using the plasma physics and electrode/gun parameters listed in Table 3.3.-I, the engineering quantities specified in Table 3.4.-I, and the economics model summarized in Tables 3.5.-I and 3.5.-II, the CSR model depicted in Fig. 2.2.-2 has been used to examine a range of net-electric powers, $P_{E}$. The resultant $C O E$ for CSRs generating net electric powers of $P_{E}=250,500,750,1,000$ MWe(net) is shown in Fig. 4.1.-1, which plots $C O E$ against the outside plasma radius or separatrix radius, $\mathrm{R}=\mathrm{R}_{\mathrm{T}}+\mathrm{r}_{\mathrm{p}}$. By varying the geometry parameter, $\mathrm{f}_{\mathrm{R}}$, in the gun/electrode scaling relationship, Eq. (3.3.-16), more of the design space becomes available. For $f_{R} \geq 1$ all minimum-COE designs are disallowed, and only small systems with very high neutron wall loading are accessible; for $f_{R} \leq 0.5$ all designs of economic and technicai interest can be accessed. Although the gun-scaling window is wide, its leverage is high. All results reported in this section display a fully opened design window (i.e., $f_{R} \leq 0.5$ ).

A prominent characteristic of Fig. 4.1.-1 is the economy-of-scale, leading to reduced unit capital and energy costs as the system capacity increases. Forcing a fixed net-electric power from a system of smaller size requires higher neutron wall loadings, as is shown in Fig. 4.1.-1; a shorter wall lifetime derived from the fixed lifetime fluence of $15 \mathrm{MWyr} / \mathrm{m}^{2}$ results. Excessively high wall loadings (i.e., $I_{W} \geq 20 \mathrm{mw} / \mathrm{m}^{2}$ ) require more than one first-wall/blanket changeout per year and a reduction in the plant factor according to the algorithm described in Table 3.5.-II (i.e., 28 days per FPC changeout). Systems with larger radii correspondingly lead to lower neutron wall loadings and much larger systems, with a modestly increasing cost because of the larger FPC mass and, hence, unit direct cost; this behavior is shown explicitly on Fig. 4.1.-2.

The relatively insensitive behavior of the unit direct cost as the system size increases is also reflected by modest increases in the FPC mass (first-wall/blanket/shield/magnets), as is shown by plotting the mass utilization in Fig. 4.1.-3. The relatively insensitive behavior, particularly for $P_{E} \geq 500$ MWe, is a consequence of the rapidly decreasing EFC coil thickness as the plasma radius increases, this latter behavior being shown in Fig. 4.1.-4. Rapidly improving magnetic coupiing as the plasma radius, $R$, becomes comparable 


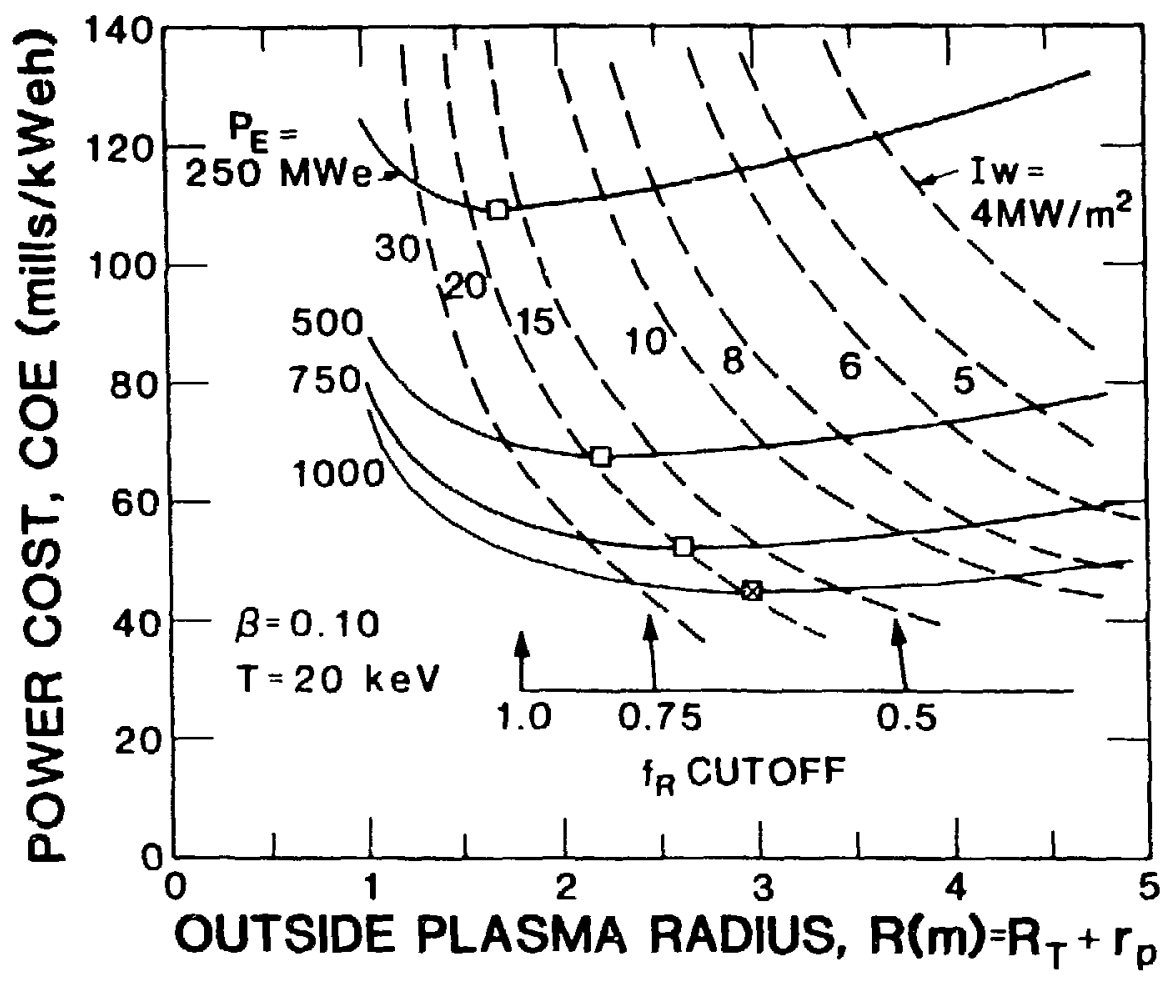

Fig. 4.1.-1. Cost of electricity (COE) for various net electric powers, $P_{E}$, versus the outside plasma radii, $R$. Also shown is the corresponding neutron wall loading.

to the EFC radius, $r_{c}$, leads to much lower values of $I_{c} / I_{\phi}$, as is shown in Fig. 4.1.-5; substantially thinner coils are required. In addition to minimizing the FPC mass as the plasma radius is increased, this tradeoff between EFC conductor thickness and coil current enforces a nearly constant recirculating power or inverse of the engineering $Q$-value, $Q_{E}$, for a given net electric power, as is shown in Fig. 4.1.-6. Power recirculated to the plasma and current-drive system decreases only a factor of two, while $P_{E}$ decreases from 1,000 to 250 MHe. The engineering Q-value then decreases substantially as the net electric power decreases. The economy of scale reflection Fig. 4.1.-1 is then accentuated by the increasing ratio of recirculated power to net electric power as $P_{E}$ decreases. Table 4.1.-I summarizes key physics, magnet, and engineering parameters for the COE-optimized $P_{E}=250,500,750$, Cnd 1,000-MHe(net) designs, and Table 4.1.-II gives the levelized cost break-down and summary for each. 


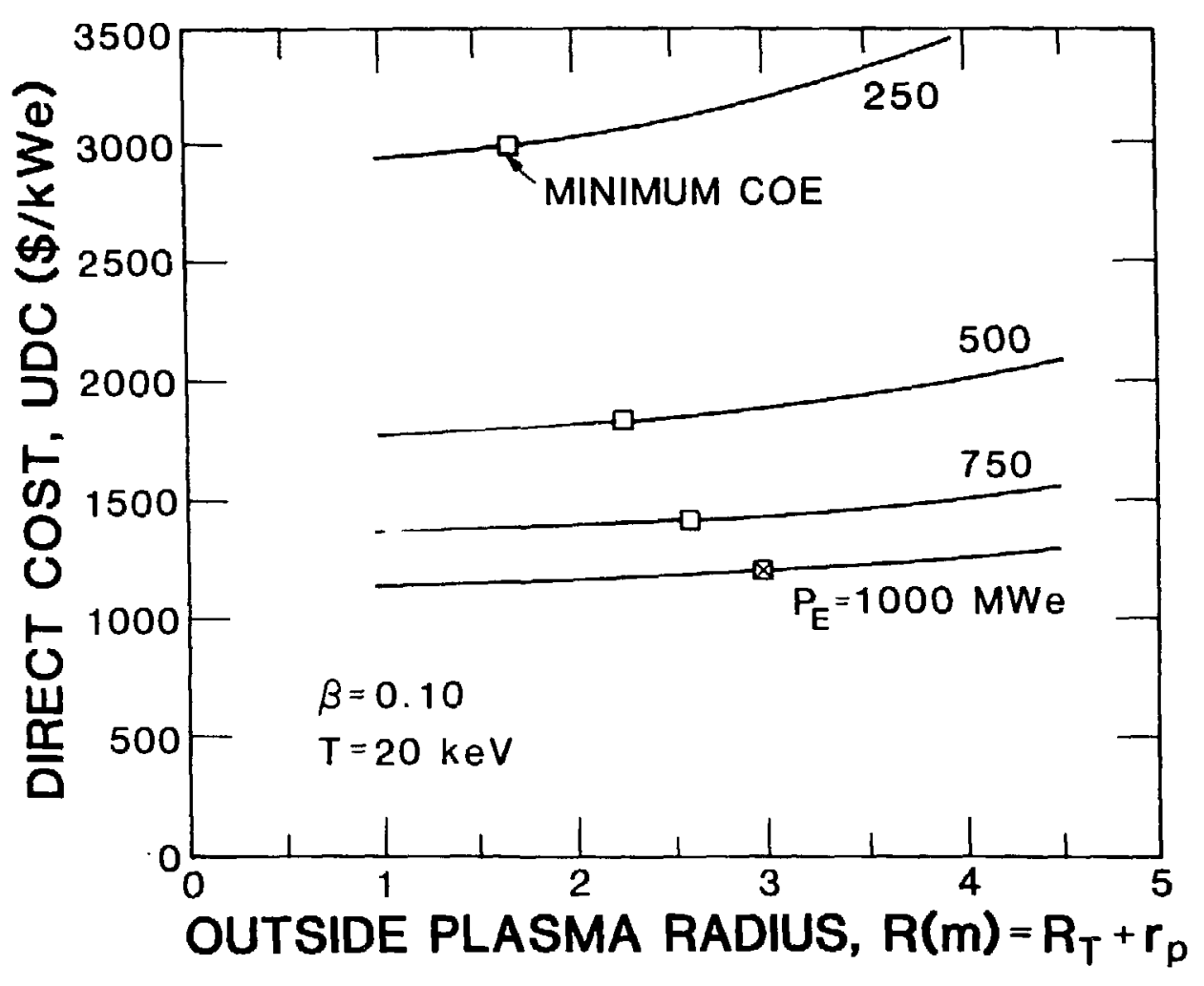

Fig. 4.1.-2. Unit direct cost versus outside plasma radius net electric power.

The economically optimal energy confinement times, $\tau_{E}$ (OPT), required for each of four net-electric powers are plotted in Fig. 4.1.-7 as a function of plasma radius. Typical energy confinement times of $0.2-0.4 \mathrm{~s}$ are needed to achieve the minimum-COE systems, with the larger systems operating with corresponding increased confinement times. Experimental scalings must yield an energy confinement time, $\tau_{E}$ (PHYS), that is greater than or equal to $\tau_{E}$ (OPT) for the design to be consistent with physics. Since no definitive confinement scalings exist for spheromaks at this time, scaling derived for reversed-field pinches $26,41,42$ has been used to compare $\tau_{E}$ (PHYS) with $\tau_{E}$ (OPT). Specifically, the electron energy confinement time is taken to be of the form

$$
\begin{aligned}
& \tau_{c e}=C_{v} I_{\phi}^{U}(0.372 R)^{2}\left(\beta_{c} / \beta\right)^{2} \\
& \tau_{p i}=4 \tau_{c e},
\end{aligned}
$$




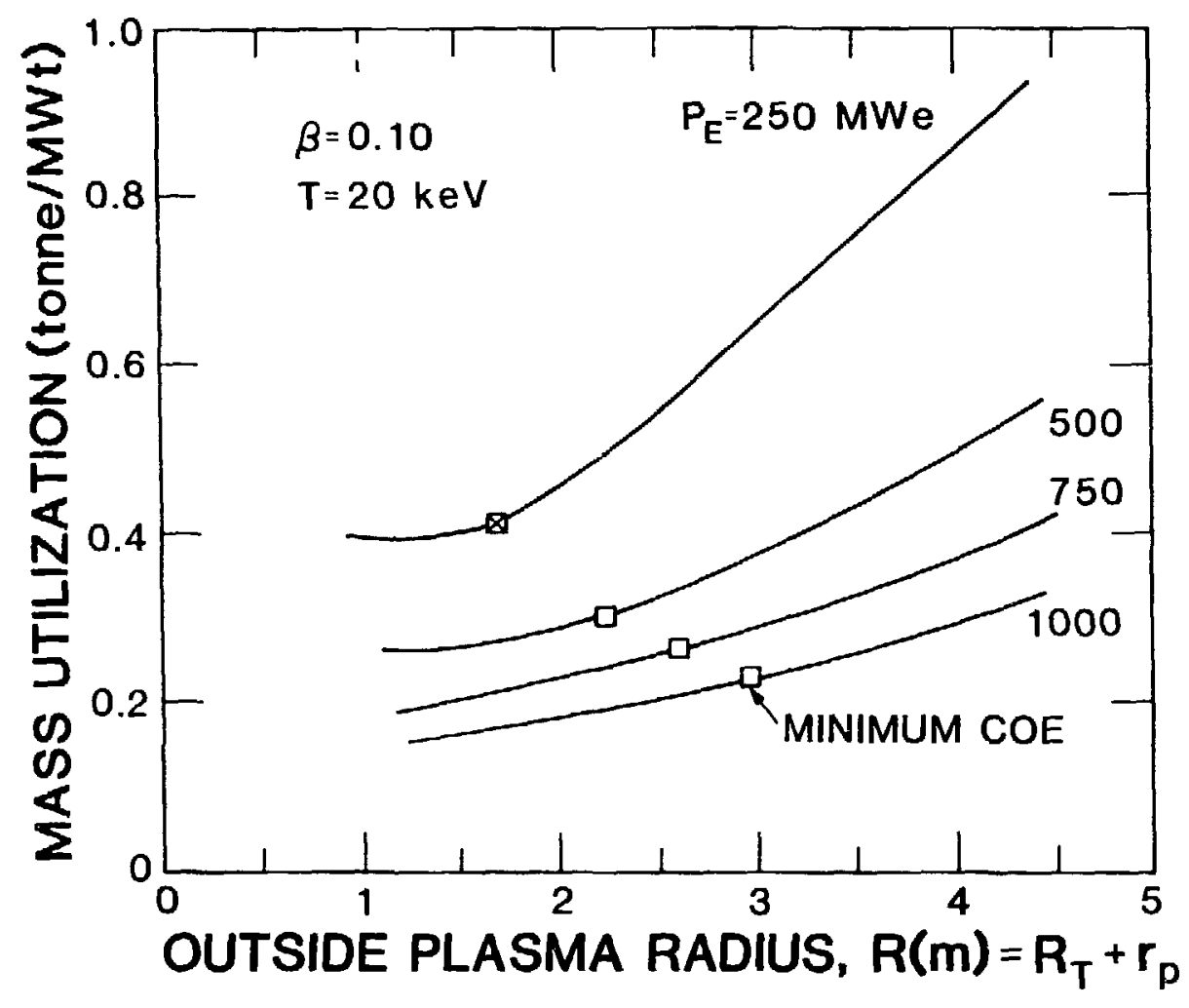

Fig. 4.1.-3. Dependence of cost optimized FPC mass utilization on plasma radius with minimum $C O E$ points indicated for each of four net-electric powers.

where the ion confinement time is $\tau_{p i}$. The electron-conduction time, $\tau_{c e}$ ' is degraded by the factor $\left(\beta_{c} / \beta\right)$ if $\beta$ exceeds a critical value $\beta_{c}$. The radial distance from the center of the plasma to the separatrix at the outer toroidal radius, $R$, is $r_{p}=0.372 R$, as is derived in $\operatorname{Sec} .3 .1$. The constant, $C_{v}$, is selected to give $\tau_{c e}=0.24 \mathrm{~ms}$ at $I_{\phi}=1.2(10)^{5} \mathrm{~A}, r_{p} \equiv 0.372 \mathrm{R}=0.2 \mathrm{~m}$, and $\beta_{c}=0.1$, for exponents $v=0.9,1.0,1.1$; these are typical ZT-40 RFP results. Using these scalings predicts very favorable plasma confinement, as is seen from Fig. 4.1.-7 [i.e., $\tau_{E}$ (OPT) $\leq \tau_{E}$ (PHYS) for the range examined]. These optimistic indications, of course, are subject to experimental verification of spheromak confinement scalings.

Finally, the plasma density, $n$, required to achieve the desired output power is plotted in Fig. 4.1.-8. Other density and current-related parameters are given in Fig. 4.1.-9 as the streaming function, $\xi=v_{D} / v_{T H} \propto I_{\phi} / N^{2} e^{1 / 2}$, and toroidal current, $I_{\phi}$, divided by the plasma line density, $N$. The plasma 


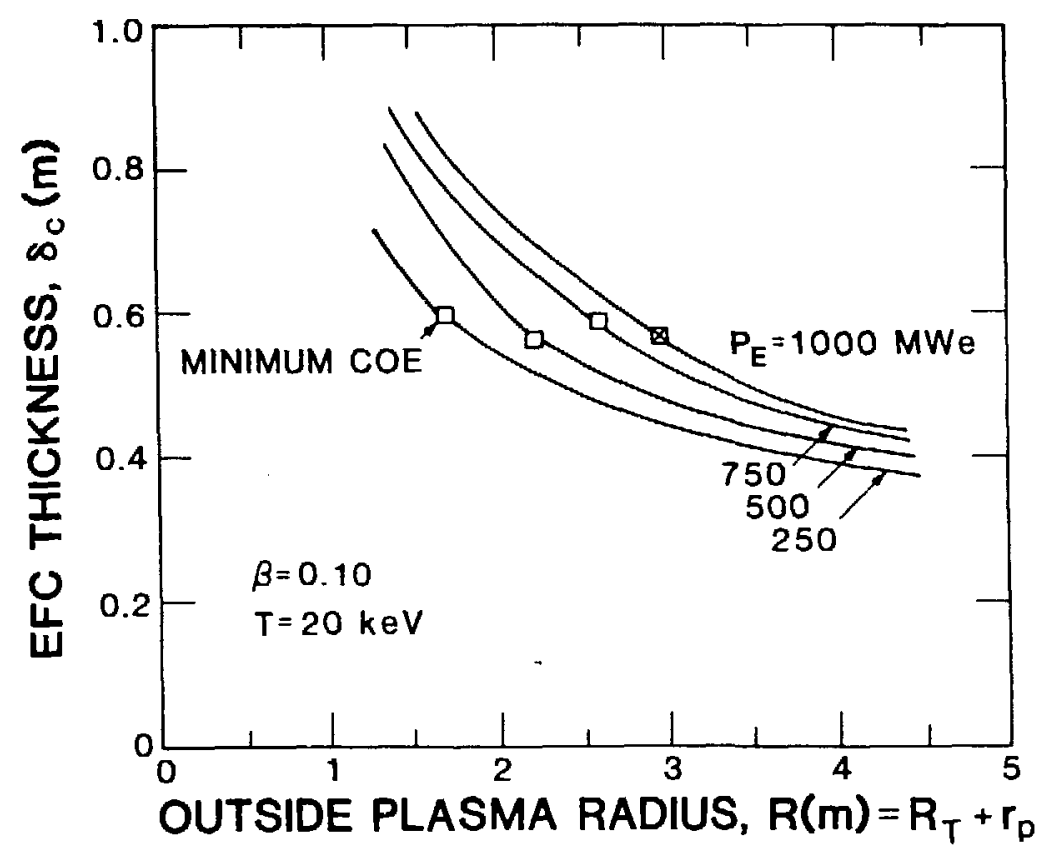

Fig. 4.1.-4. Dependence of EFC thickness on plasma radius, with minimum-COE points indicated for each of four net-electric powers.

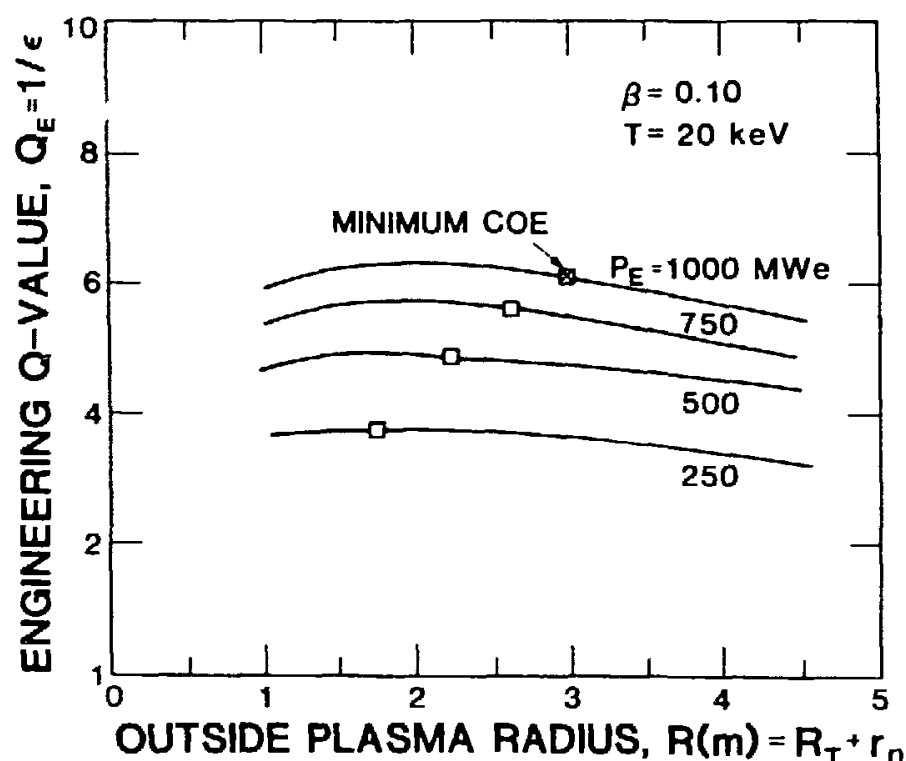

Fig. 4.1.-5. Dependence of plasma and EFC currents on plasma radius, with minimum-COE points indicated for each of four net-electric powers. 


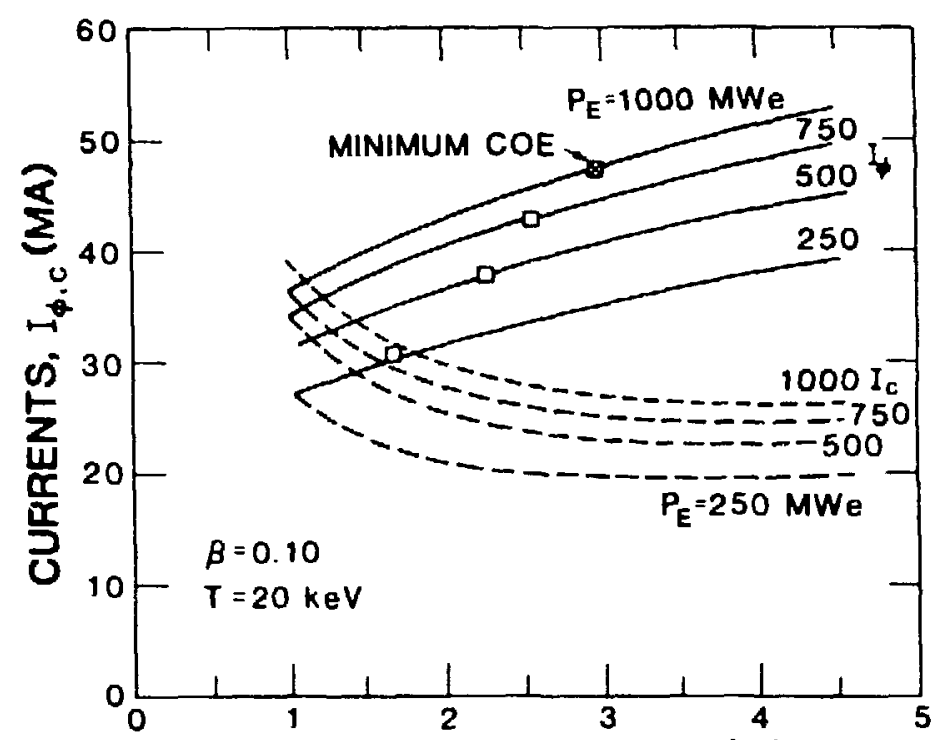

OUTSIDE PLASMA RADIUS, $R(m)=R_{T}+r_{p}$

Fig. 4.1.-6. Dependence of cost-optimized engineering Q-value on plasma radius with minimum-COE points indicated for each of four net-electric powers.

streaming function relates the electron drift velocity resulting from current flow to the electron thermal velocity.

\subsection{Major Variable Sensitivities}

The basecase summarized in Tables 4.1.-I and 4.1.-II is considered the optimal design configuration for the fixed parameter assumed. As illustrated in Fig. 2.2.-2, the complete specification of an optimal design requires searches of the aspect ratio, $L / R$, and the operating plasma temperature, $T$. Theoretical equilibrium/stability formulations ${ }^{7}$ and present experimental configur : ions ${ }^{58}$ indicate aspect ratios of $L / R=1.25$ may be nearly optimal, alt ugh the variable is subjected to a design sensitivity analysis. An optimum plasma temperature is expected, wherein low values of $T$ produce excessive plasma resistive dissipation while high values reduce the plasma fusion power density for a given pressure. An optimal operating temperature of $20 \mathrm{keV}$ is typically found, with only a modest variation in COE being observed for temperatures in the range $T=10$ to $30 \mathrm{keV}$.

The optimal $P_{E}=1000$-MWe design described in Table 4.1.- $I$ is then subjected to a design sensitivity analysis with $T=20 \mathrm{keV}$ and $\mathrm{L} / \mathrm{R}=1.25$. Seven key variables were identified for sensitivity analyses: helicity injection 
TABLE 4.1.-IA.

SUMMARY OF PLASMA PARAMETERS FOR THE FULLY

COE-OPTIMIZED DESIGNS SHOWN ON FIG. 4.1.-1

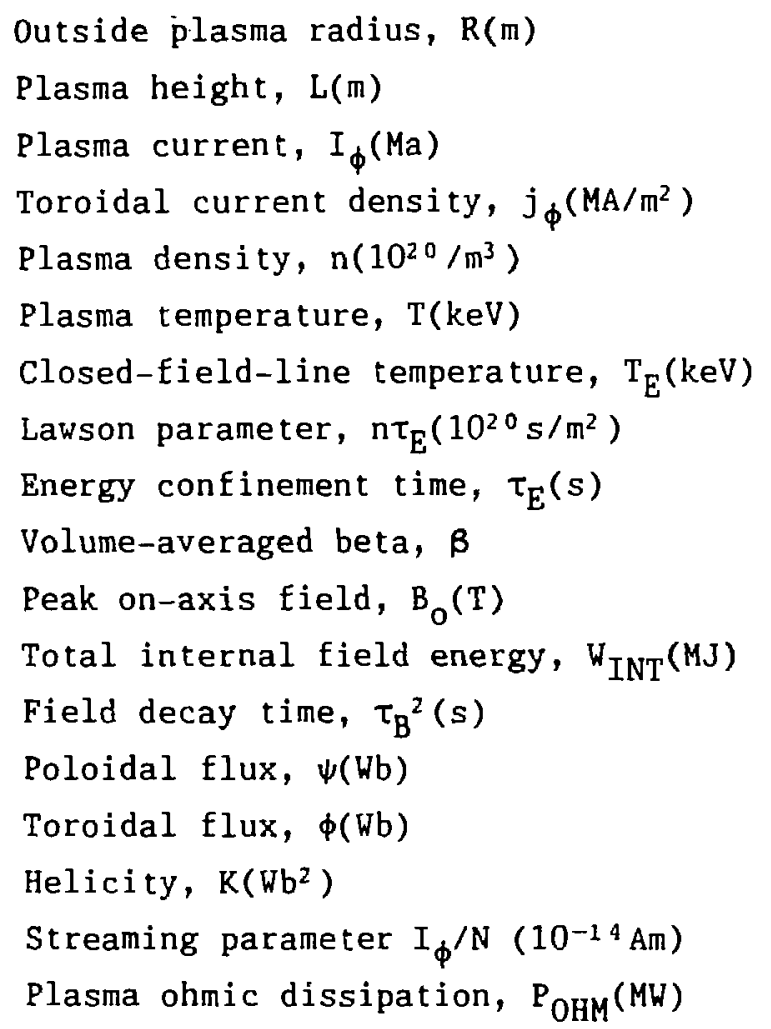

$\begin{array}{rrrr}1.70 & 2.22 & 2.60 & 2.98 \\ 2.12 & 2.78 & 3.25 & 3.72 \\ 30.03 & 37.49 & 42.73 & 47.29 \\ 8.35 & 6.07 & 5.06 & 4.28 \\ 2.80 & 2.54 & 2.41 & 2.26 \\ 20.00 & 20.00 & 20.00 & 20.00 \\ 0.50 & 0.50 & 0.50 & 0.50 \\ 0.96 & 0.96 & 0.96 & 0.96 \\ 0.34 & 0.38 & 0.40 & 0.43 \\ 0.10 & 0.10 & 0.10 & 0.10 \\ 13.93 & 13.27 & 12.94 & 12.51 \\ 343.50 & 701.10 & 1,065.35 & 1,494.39 \\ 25.70 & 43.99 & 60.04 & 78.55 \\ 21.42 & 35.03 & 46.69 & 59.17 \\ 13.97 & 22.84 & 30.45 & 38.58 \\ 319.62 & 854.59 & 1,518.16 & 2,430.16 \\ 2.99 & 2.39 & 2.10 & 1.90 \\ 13.37 & 15.94 & 17.75 & 19.02\end{array}$

efficiencies, $\varepsilon_{K}$; volume-average plasma beta, $\beta$; edge-plasma temperature, $\mathrm{T}_{\mathrm{E}}$; Eusion-neutron first-wall loading, $I_{w}$, blanket/shield thickness, $\triangle b$; FPC radiation life, $I_{w} \tau$; and arc voltage drop at the electrode-gun divertor, $\nabla_{A}$. Figure 4.2.-1 summarizes the COE sensitivity to the net electric power, $P_{E}$, and most of the above-mentioned variables. over the range of $P_{E}$ studied (250-1,500 MWe), the ohmic losses in the plasma do not dominate the recirculating power, giving a weak dependence of $\operatorname{COE}$ on $\varepsilon_{\mathrm{K}}$ and $\mathrm{T}_{E}$; the $\mathrm{T}_{E}$ dependence is not shown, but the impact on $\mathrm{COE}$ is $\leq 1 \%$ for the range indicated. Increases in $V_{A}$ generally cause the minimum-COE designs to occur at a larger value of total gun voltage, $V_{g}$, in order to minimize gun current and associated 
SUMMARY OF EFC PARAMETERS FOR THE FULLY

COE-OPTIMIZED DESIGNS SHOWN ON FIG. 4.1.-1

\begin{tabular}{lll}
\multicolumn{4}{c}{$\mathrm{P}_{\mathrm{E}}(\mathrm{MWe})$} \\
\hline $250 \quad 500$ & 750 & 1,000
\end{tabular}

Coil thickness, $\delta_{c}(m)$

Average minor radius of coil, $r_{c}(m)$

Mass of coil, $\mathrm{M}_{\mathrm{EFC}}$ (tonne)

Magnetic-field level at coil, $B_{c}(T)$

Magnetic-field level at plasma surface, B(T)

Poloidal-coil current, $I_{c}(M A)$

Poloidal current density, $j_{c}\left(M A / m^{2}\right)$

Maximum energy stored in coil, $W_{B}(M J)$

Ohmic dissipation during burn, $\mathrm{P}_{E F C}(M W)$

Volumetric EFC heating, $\mathrm{P}_{\mathrm{EFC}} / \mathrm{V}_{\mathrm{EFC}}\left(\mathrm{MW} / \mathrm{m}^{3}\right)$
0.59

0.56

0.59

3.20

3.59

608.48

$357.92 \quad 465.05$

3.01

2.79

2.70

5.35

5.21

23.82

25.68

6.15

5.74

$787.87 \quad 1232.82$

1682.23

70.52

0.85

0.57

3.96

708.79

2.55

5.04

26.97

5.75

2135.69

$45.47 \quad 62.00 \quad 70.52 \quad 84.50$

0.92

0.97

arc losses, thereby giving a weaker dependence of $\mathrm{COE}$ on $V_{\mathbf{g}}$ than originally expected; the $V_{g}$ dependence is also $\leq 1 \%$ and is not shown on Fig. 4.2.-1. The general reduction in $\mathrm{COE}$ sensitivity to key physics and engineering assumptions is characteristic of the compact reactor approaches; the FPC cost is only a few percent of the total direct cost, thereby decoupling the FPC from the overall cost equation.

\subsection{Preliminary Considerations for Fusion-Power-Core Layout}

The fusion power core (FPC) that served as a basis for the results reported in the previous Secs. 4.1. and 4.2. was based on a self-cooled $\mathrm{Pb}_{83} \mathrm{Li}_{17}$ blanket and $a \sim 0.1-m$-thick steel structural shield that protected the resistive copper EFCs. This blanket/shield system is similar to that examined for the CRFPR in Ref. 25. Although the present scoping study of the CSR did not permit neutronic, magnetics, thermal-hydraulic, structural, and maintenance designs or the associated FPC integration studies to be performed, the FPC dimensions given on Tables 4.1.-I are sufficient for the construction of an illustrative isometric drawing of the FPC envisaged for the CSR. Figure 4.3.-1 gives such a sketch for the $P_{E}=1,000-M W e(n e t)$ design summarized on Table 4.1.-I. 
SUMMARY OF GUN/ELECTRODE PARAMETERS FOR THE FULLY COE-OPTIMIZED DESIGNS SHOWN ON FIG. 4.1.-1

\begin{tabular}{|c|c|c|c|c|}
\hline & \multicolumn{4}{|c|}{$P_{E}($ MWe $)$} \\
\hline & 250 & 500 & 750 & 1,000 \\
\hline Thermal load, $\mathrm{I}_{\mathrm{QW}}\left(\mathrm{MW} / \mathrm{m}^{2}\right)$ & 5.00 & 5.00 & 5.00 & 5.00 \\
\hline Inner radius, $r_{g i}(m)$ & 0.95 & 1.24 & 1.45 & 1.66 \\
\hline Outer radius, $r_{g o}(m)$ & 1.42 & 1.86 & 2.17 & 2.49 \\
\hline Length, $\ell_{g}(m)$ & 2.29 & 3.15 & 3.85 & 4.38 \\
\hline Divertor channel length $(\mathrm{m})$ & 4.96 & 6.58 & 7.77 & 8.88 \\
\hline Temperature at electrode, $\mathrm{T}_{\mathrm{D}}(\mathrm{eV})$ & 3.00 & 3.00 & 3.00 & 3.00 \\
\hline Factor times Taylor current, $1 / \varepsilon_{\mathrm{K}}$ & 1.50 & 1.50 & 1.50 & 1.50 \\
\hline Inner (plasma) flux radius, $r_{0}(m)$ & 0.04 & 0.05 & 0.06 & 0.06 \\
\hline Fraction of poloidal flux diverted, $100 \varepsilon$ & 0.34 & 0.31 & 0.29 & 0.28 \\
\hline Plasma edge temperature, $\mathrm{T}_{\mathrm{e}}(\mathrm{eV})$ & 40.44 & 44.09 & 46.42 & 48.32 \\
\hline Dissipative arc voltage, $\mathrm{V}_{\mathrm{gA}}(\mathrm{V})$ & 0.00 & 0.00 & 0.00 & 0.00 \\
\hline Total voltage, $v_{g}(v)$ & 91.04 & 94.70 & 96.91 & 98.31 \\
\hline Current, $I_{g}(M A)$ & 0.23 & 0.26 & 0.28 & 0.29 \\
\hline Divertor channel dissipation, $\mathrm{P}_{\mathrm{gR}}(\mathrm{MW})$ & 1.17 & 0.99 & 0.91 & 0.84 \\
\hline Total input power, $\mathrm{P}_{\mathrm{g}}(\mathrm{MW})$ & 21.14 & 24.92 & 27.55 & 29.40 \\
\hline
\end{tabular}

It is emphasized that the layout suggested in Fig. 4.3.-1 has been generated to give a better perspective of the Table 4.1.-I systems-code results, and a detailed engineering design remains to be performed. This layout is based on a - 0.6-0.7-m-thick self-cooled PbLi blanket with 3-channel flow parallel to the poloidal field. The $\sim 0.10$-m-thick structural steel shield that forms and supports the main confinement chamber is also cooled with a slip stream of PbLi. It is presumed that a low-radiation plasma deposits most of the alphaparticle and ohmic-heating power onto open field lines and into the co-axial divertor/gun electrode system, which is shown in Fig. 4.3. 1 as located atop of the main reaction chamber. The primary PbLi coolant enters at the top of the reaction chamber and flows poloidally downward and exits at the bottom. Four main coolant loops are indicated. A part of the PbLi inlet flow to the blanket $(\sim 2,842 \mathrm{MWt})$ is diverted to cool the outer electrode of the divertor/gun 
SUMMARY OF KEY ENGINEERING PARAMETERS FOR THE FULLY COE-OPTIMIZED DESIGNS SHOWN ON FIG. 4.1.-1

\begin{tabular}{llll}
\multicolumn{4}{c}{$\mathrm{P}_{E}(\mathrm{MWe})$} \\
\hline 250 & 500 & 750 & 1,000
\end{tabular}

Ohmic Q-value, $Q_{p}$

Total thermal power, $\mathrm{P}_{\mathrm{TH}}$ (MW)

Thermal conversion efficiency, $n_{T H}$

Recirculating power fraction, $\varepsilon=1 / 0_{E}$

Plant efficiency, $n_{\mathrm{p}}=n_{\mathrm{TH}}(1-\varepsilon)$

14.1-MeV neutron loading, $I_{w}\left(M W / \mathrm{m}^{2}\right)$

14.1-MeV blanket multiplication, $M_{N}$

First-wall radius, $R(m)$

Minor radius of system, $r_{p}(m)$

Fusion-power-core mass, $\mathrm{M}_{\mathrm{FPC}}$ (tonne)

System power density, $\mathrm{P}_{\mathrm{TH}} / \mathrm{V}_{\mathrm{FPC}}\left(\mathrm{MW} / \mathrm{m}^{3}\right)$

Mass power density, MPD(kWe/tonne)

Mass utilization, $\mathrm{MU}=\mathrm{M}_{\mathrm{FDC}} / \mathrm{P}_{\mathrm{TH}}$ (tonne/MWt)

Blanket/shield thickness, $\Delta b(m)$

Mass of first-wall/blanket (tonne)

Blanket power density $\left(\mathrm{MWt} / \mathrm{m}^{3}\right)$ $\begin{array}{llll}14.59 & 20.74 & 26.57 & 30.53\end{array}$

973. $1,803.2,605.3,416$.

$\begin{array}{llll}0.35 & 0.35 & 0.35 & 0.35\end{array}$

$\begin{array}{llll}0.27 & 0.21 & 0.18 & 0.16\end{array}$

$\begin{array}{llll}0.26 & 0.28 & 0.29 & 0.29\end{array}$

$\begin{array}{llll}17.36 & 18.74 & 19.81 & 19.82\end{array}$

$\begin{array}{llll}1.30 & 1.30 & 1.30 & 1.30\end{array}$

$\begin{array}{llll}1.70 & 2.22 & 2.60 & 2.98\end{array}$

$\begin{array}{llll}2.99 & 3.48 & 3.89 & 4.25\end{array}$

$\begin{array}{llll}395.13 & 523.62 & 685.30 & 806.39\end{array}$

$\begin{array}{llll}8.69 & 10.20 & 10.59 & 20.65\end{array}$

$632.38 \quad 954.801,095.601,240.10$

$\begin{array}{llll}0.41 & 0.29 & 0.26 & 0.23\end{array}$

$\begin{array}{llll}0.70 & 0.70 & 0.70 & 0.70\end{array}$

$\begin{array}{llll}37.20 & 58.57 & 76.82 & 97.60\end{array}$

$\begin{array}{llll}26.16 & 30.78 & 33.91 & 35.00\end{array}$

system. As shown in Fig. A-4 for $\varepsilon=0.01$ diverted poloidal flux, $f_{i} \simeq 0.18$ of the alpha-particle $\left(P_{\alpha}=551 \mathrm{MW}\right)$ and ohmic $\left(\mathrm{P}_{\Omega \mathrm{p}}=19 \mathrm{MW}\right)$ plasma powers are received by the outer electrode $\left[\left(1-f_{i}\right)\left(P_{\Omega p}+P_{\alpha}\right) \simeq 467 \mathrm{MH}\right]$, with $f_{i}\left(P_{\Omega p}+P_{\alpha}\right)=103$ MWt being deposited into the inner electrode as sensible heat. Nuclear heating in the divertor/gun electrode system has not been determined and is not considered at the present level of analysis. The inlet and outlet headering for the blanket and outer-electrode systems on Fig. 4.3.-1 has been sized for a 400-K PbLi temperature change, a flow velocity of $\sim 1 \mathrm{~m} / \mathrm{s}$ [flow area $\left.\left(\mathrm{m}^{2}\right) \simeq \mathrm{P}_{\mathrm{TH}}(\mathrm{MWt}) / 632\right]$, and four separate coolant loops. 
COST SUMMARY FOR COE-OPTIMIZED $P_{E}=250-M W e(n e t)$ DESIGN

ACC. NO

ACCOUNT TITLE

20. Land and land rights

(M\$, 1.980)

21. Structures and site facilities

3.30

22. Reactor plant equipment 260.94

23. Turbine plant equipment 113.34

24. Electric plant equipment

25. Miscellaneous plant equipment 88.77

26. Special materials

90. Total reactor direct capital cost 10.32

91. Construction facilities, equipment and services (10\%)

92. Engineering and construction management services (8\%)

93. Other costs (5\%) 610.50 61.05 48.84

94. Interest during construction ( $10 \% / y)$ 30.53

95. Escalation during construction ( $5 \% / y)$ 188.78

99. Total reactor capital cost 116.39 $1,055.78$

Thermal power, $\mathrm{P}_{\mathrm{TH}}(\mathrm{MWt})$

Direct capital cost, $\operatorname{UDC}(\$ / \mathrm{kWe})$

Gross electric power, $\mathrm{P}_{\mathrm{ET}}$ (MWe)

Direct investment $\cos t(\$ / \mathrm{kWe})$

Net electric power, $P_{E}$ (MWe)

Total investment cost ( $\$ / k W e)$

Engineering $Q$-value, $\mathrm{Q}_{\mathrm{E}}=1 / \varepsilon$

Capital return $15 \%$ (mills/kWeh)

Plant factor, $\mathrm{p}_{\mathrm{f}}$

Operating 2\% (mills/kWeh)

Replacement (mills/kWeh)

Energy cost, COE(mills/kWeh)
973.02

$2,441.88$

340.56

$3,003.51$

250.01

$4,222.93$

3.76

95.28

0.76

11.53

2.59

109.40

Because the inner electrode must operate at a voltage $V_{g} \simeq 100 \mathrm{~V}$ (Table 4.1.-I) and must deliver $I_{g}=0.3 \mathrm{MA}$ and $P_{g} \simeq 30 \mathrm{MW}$, the design of this cooling system must be considered separately. Because of the relatively high heat fluxes being considered $\left(\sim 5 \mathrm{MW} / \mathrm{m}^{2}\right)$, it seems unlikely that the voltage 
COST SUMMARY FOR COE-OPTIMIZED $\mathrm{P}_{\mathrm{E}}=500$-MWe(net) DESIGN

ACC. NO

20.

21.

Land and land rights

ACCOUNT TITLE

22.

Structures and site facilities

Reactor plant equipment

23. Turbine plant equipment

24. Electric plant equipment

25. Miscellaneous plant equipment

26. Special materials

90.

Total reactor direct capital cost

91.

Construction facilities, equipment and services (10\%)

92.

Engineering and construction management services (8\%)

93. 0ther costs ( $5 \%)$

94. Interest during construction $(10 \% / y)$

95. Escalation during construction ( $5 \% / \mathrm{y}$ )

99. Total reactor capital cost
(MS, 1980)

3.30

262.28

175.54

141.50

99.83

41.83

16.11

740.39

74.04

59.23

37.019

228.58

141.15

$1,280.41$

Thermal power, $\mathrm{P}_{\mathrm{TH}}(\mathrm{MWt})$

1803.05

Direct Capital cost, UDC ( $\$ / \mathrm{kWe})$

$1,480.86$

Gross electric power, $\mathrm{P}_{\mathrm{ET}}$ (Mvie)

Direct Investment cost ( $\$ / \mathrm{kWe}$ )

$1,821.46$

Net electric power, $\mathrm{P}_{\mathrm{E}}$ (MWe)

499.97

Total investment cost ( $\$ / \mathrm{kWe}$ )

$2,560.97$

$1 /$ recirculating power fraction, $0_{E}=1 / \varepsilon$

4.81

Capital return 15\% (mills/kWeh)

57.78

Plant factor, $\mathrm{P}_{\mathrm{f}}$

0.76

Operating 2\% (mills/kWeh)

6.99

Replacement (mills/kWeh)

2.20

Power cost, $\operatorname{COE}(\mathrm{mill} / \mathrm{s} / \mathrm{kWeh})$

66.98

stand-off can be placed between the heat source and the inner electrode cooling system; all or part of the inner-electrode cooling system, therefore, would operate at a floating potential of $\sim 100 \mathrm{~V}$. Leakage currents and associated 
COST SUMMARY FOR COE-OPTIMIZED $P_{E}=750-$ MWe(net) DESIGN

ACC. NO

20. Land and land rights

ACCOUNT TITLE

21. Structures and site facilities

22. Reactor plant equipment

23. Turbine plant equipment

24. Electric plant equipment

25. Miscellaneous plant equipment

20. Special materials

90. Total reactor direct capital cost

91. Construction facilities, equipment and services (10\%)

92. Engineering and construction management services (8\%)

93. Other costs (5\%)

94. Interest during construction $(10 \% / y)$

95. Escalation during construction $(5 \% / y)$

99. Tetal reactor capital cost

Thermal power, $\mathrm{P}_{\mathrm{TH}}$ (MWt)

Direct Capital cost, UDC ( $\$ / k W e)$

Gross electric power, $\mathrm{P}_{\mathrm{ET}}$ (MWe)

Direct Investment cost ( $\$ / \mathrm{kWe})$

Net electric power, $P_{E}$ (MWe)

Total investment cost ( $\$ / \mathrm{kWe}$ )

$1 /$ recirculating power fraction, $Q_{E}=1 / \varepsilon$

Capital return $15 \%$ (mills/kWeh)

Plant factor, $\mathrm{P}_{\mathrm{f}}$

Operating 2\% (mills/kWeh)

Replacement (mills/kWeh)

Power cost, $\operatorname{COE}(m i l l s / k W e h)$
(M\$, 1980)

3.30

263.20

236.94

183.71

110.53

41.83

21.05

860.55

86.06

68.84

43.03

265.68

164.07

$1,488.23$
2605.38

$1,147.43$

911.83

$1,411.34$

749.98

$1,984.34$

5.63

44.78

0.76

5.42

2.04

52.24

resistive power losses along the inner-electrode coolant headers/manifolds and to the (presumably) grounded heat exchanger or point of juncture with the other parts of the main coolant loop become a concern. Use of an insulating coolant 
COST SUMMARY FOR COE-OPTIMIZED $P_{E}=1000-M W e(n e t)$ DESIGN

ACC. NO

ACCOUNT TITLE

(MS, 1980)

20. Land and land rights

3.30

21. Structures and site facilities

263.77

22. Reactor plant equipment

297.56

23. Turbine plant equipment

223.04

24. Electric plant equipment

121.33

25. Miscellaneous plant equipment

26. Special materials

26.67

90. Total reactor direct capital cost

977.70

91. Construction facilities, equipment and services ( $10 \%)$

97.77

92. Engineering and construction management services ( $8 \%$ )

78.22

93. 0 ther costs (5\%)

48.89

94. Interest during construction (10\%/y)

301.85

95. Escalation during construction ( $5 \% / y)$

186.40

99.

Total reactor capital cost

$1,690.82$

Thermal power, $\mathrm{P}_{\mathrm{TH}}$ (HWt)

Direct Capital cost, UDC ( $\$ / \mathrm{kWe}$ )

Gross electric power, $\mathrm{P}_{E T}$ (MVe)

Direct Investment $\operatorname{cost}(\$ / \mathrm{kWe})$

Net electric power, $\mathrm{P}_{E}$ (MWe)

Total investment cost $(\$ / \mathrm{kHe})$

$1 /$ recirculating power fraction, $Q_{E}=1 / \varepsilon$

Capital return $15 \%$ ( $\mathrm{mills} / \mathrm{kWeh}$ )

Plant factor, $\mathrm{P}_{\mathrm{f}}$

Operating 2\% (mills/kWeh)

Replācement (mills/kWeh)

Pover cost, $\operatorname{COE}(\mathrm{mills} / \mathrm{kWeh})$
$3,415.95$

977.72

$1,195.59$

$1,202.59$

999.99

$1,690.84$

6.11

38.16

0.76

4.62

1.94

44.72

like He or pressurized water would restrict these current leakage paths to the coolant-tube walls per se and the associated power loss could be made an acceptable small fraction of $\mathrm{P}_{\mathrm{g}}$. Helium, however, is probably unacceptable from 


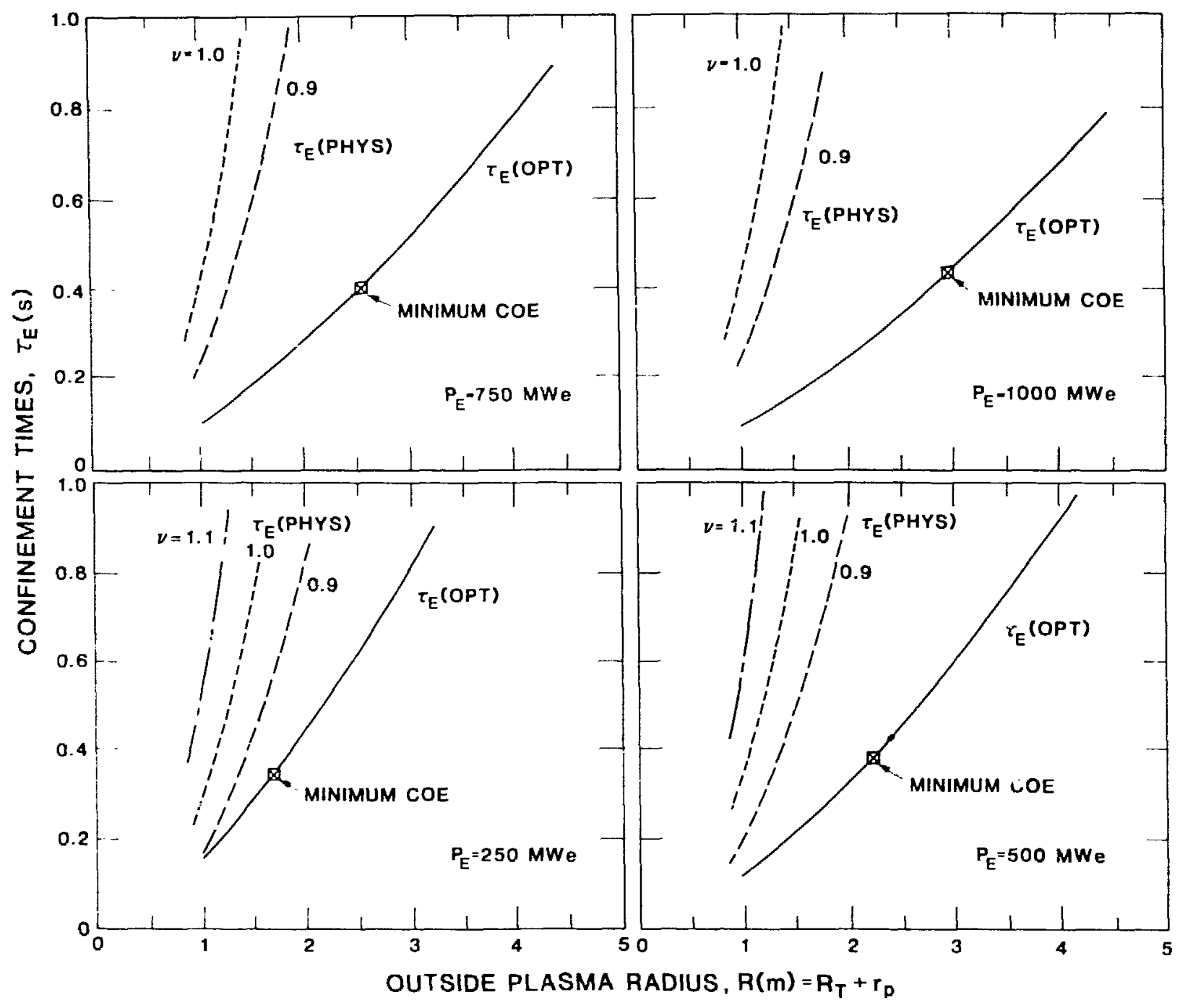

Fig. 4.1.-7. Economically optinal energy confinement times, $\tau_{E}(O P T)$, plotted against reversed-field pinch $26,41,42$ experimental scalings of the form $\tau_{c e} \propto I_{\phi}^{U} r_{p}^{2}\left(\beta_{c} / \beta\right)^{2}$ where; $v=0.9,1.0,1.1$ and $\beta_{c}=0.1$. 


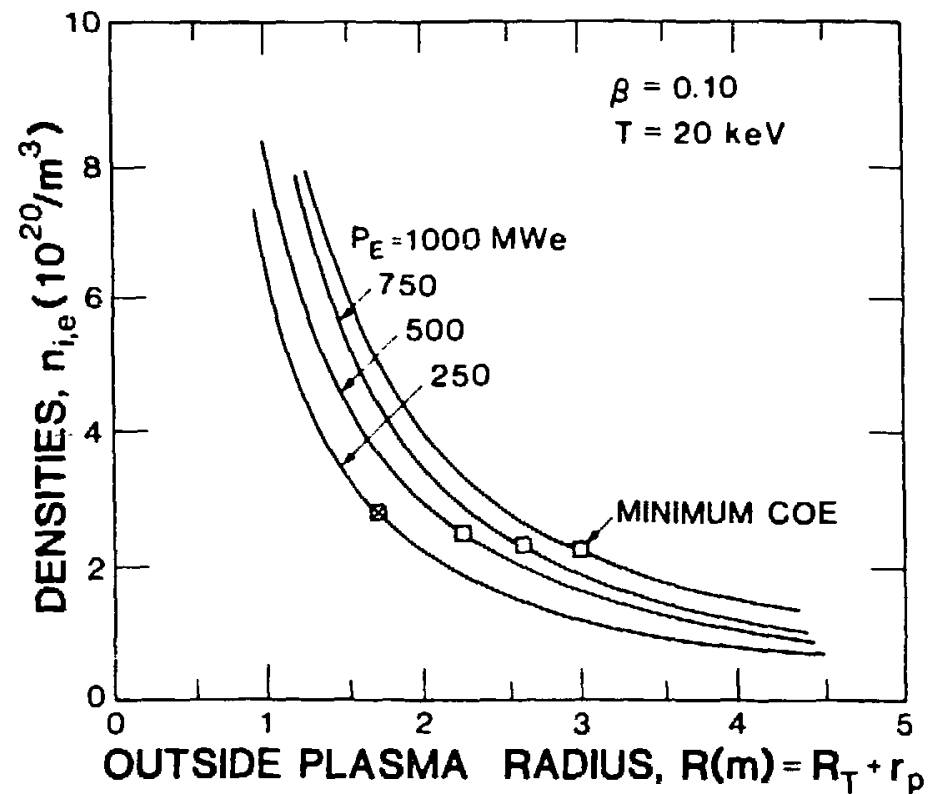

Fig. 4.1.-8. Dependence of average plasma density of plasma radius, with minimum-COE points indicated for each of four net-electric powers.

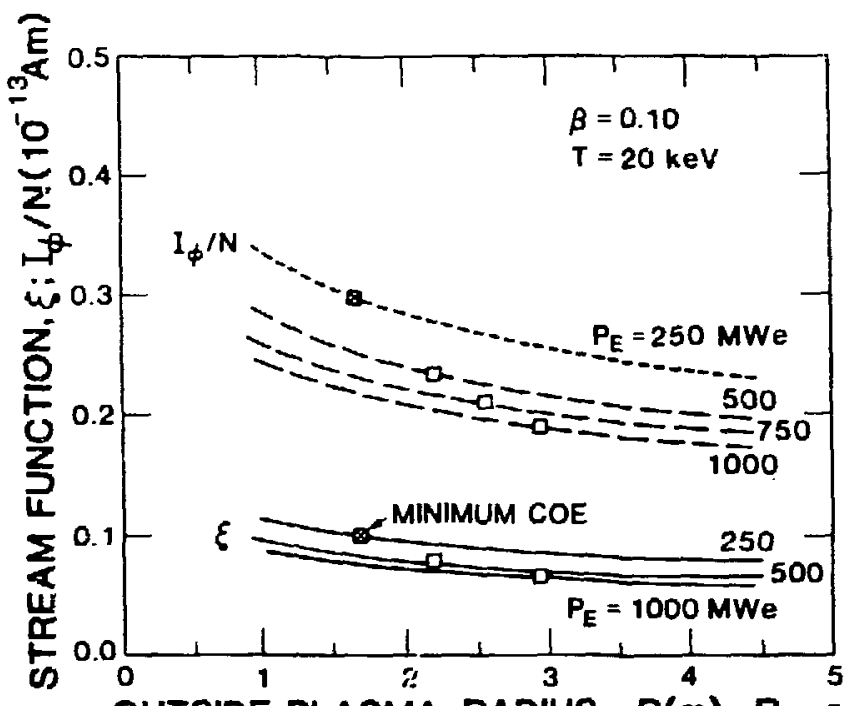

OUTSIDE PLASMA RADIUS, $R(m)=R_{T}+r_{p}$

Fig. 4.1.-9. Dependence of $I_{\phi} / N$ and streaming parameter on plasma radius, with minimum-COE points indicated for each of four net-electric powers. 


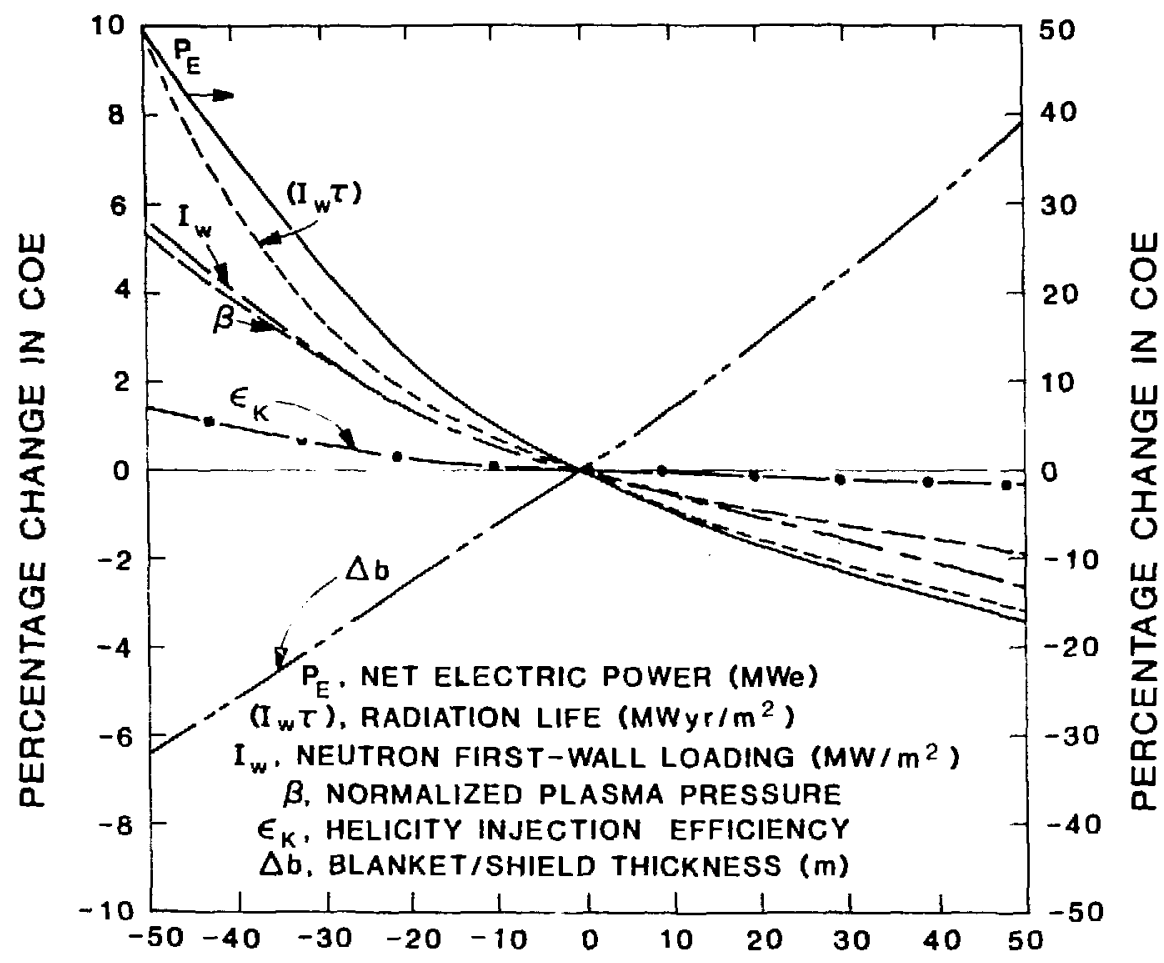

PERCENTAGE CHANGE IN PHYSICS OR DESIGN VARIABLE

Fig. 4.2.-1. Sensitivity of cost of electricity to key physics and engineering parameters.

the viewpoint of high heat flux, and the use of pressurized water adds the complexity associated with a dual-media coolant system, 25 as well as returning to the problem of chemical reactivity with PbLi. One option is to cool the inner electrode also with flowing PbLi and simply isolate the voltage $v_{g}$ by a sufficiently long run of header piping of sufficiently small diameter. For a PbLi resistivity of $\sim 10^{-6}$ ohm $\mathrm{m}$, which is about the same as for the stainlesssteel pipe, a flow velocity of $5 \mathrm{~m} / \mathrm{s}$, a power handling requirement of $f_{i}\left(P_{\alpha}+P_{\Omega p}\right)=103 \mathrm{MW}$, and a PbLi temperature change of $\sim 400 \mathrm{~K}$, the power associated with leakage current amounts to $\mathrm{P}_{\mathrm{L}}(\mathrm{MW})=629 / \mathrm{l}_{\mathrm{L}}$, where $\ell_{\mathrm{L}}$ is the length of each PbLi inlet and outlet header. of this power loss, the fraction $\eta_{\mathrm{TH}}$ is recovered by the thermal cycle. For this case, the header diameter of a single inner-electrode coolant loop is $0.2 \mathrm{~m}$, and for $P_{L} \leq P_{g}$, the length of headering is ${ }^{l} \mathrm{~L} \geq 21 \mathrm{~m}$; this length is equal to the height of each of four PWR steam generators for a 1,000-MWe(net) plant and is comparable to the normal pipe runs expected for a power plant of this capacity. 


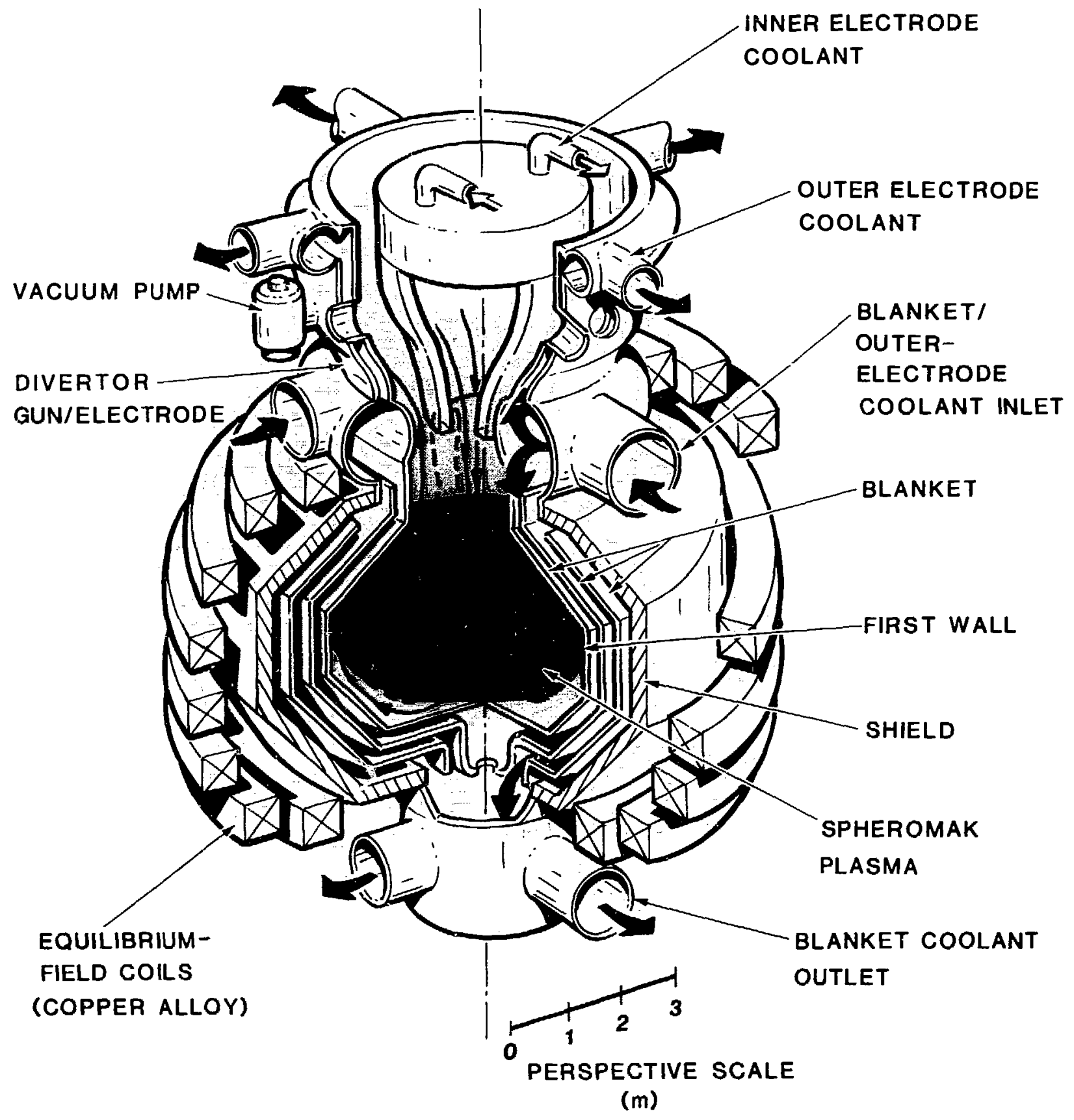

Fig. 4.3.-1. Schematic drawing of the $P_{E}=1,000-M W e(n e t)$ FPC described on Table 4.1.-I for the PbLi-self $\mathrm{f}$-conled basecase. 
In summary, the preliminary estimates reflected in the sketch given in Fig. 4.3.-1 indicate a single-media and efficient PbLi coolant may be possible for the CSR. Detailed configuration studies, however, require a better resolution of the spatial distribution of surface-heat fluxes and nuclear heating in the divertor/gun-electrode system, as well as feasibility of operating the spheromak plasma in a low-radiation mode needed to have reduced first-wall heat fluxes and high FPC power density. In this connection, a broader range of electrode configurations (i.e., off-axis) should be considered by future work. 


\section{SUMMARY AND CONCLUSIONS}

\subsection{Design Summary and Comparisons}

Table 5.1.-I summarizes the essential elements of the CSR for a range of net-electric powers. Comparison of the $P_{E}=1000-M W e$ design has been $g$ iven in Table 1.1.-I and Fig. 1.2.-2, whereas Table 1.1.-III gives a comparison with the other promising compact, resistive-coil approaches, which include the compact RFP reactor (CRFPR ${ }^{2}$ ), and the low-aspect-ratio Advanced Tokamak Reactor based on the Spherical Torus (ATR/ST ${ }^{4}$ ). Although most compact approaches tend to optimize with FPCs that exceed the 100-200 kWe/tonne threshold target suggested by generic reactor studies,15,91 the general dependence of COE on plant

\section{TABLE 5.1.-I}

SPHEROMAK FPC DESIGN-POINT SUMMARY

Net electric power; $P_{E}$ (MWe)

Fusion neutron first-wall loading,

$$
I_{W}\left(M W / m^{2}\right)
$$

Total thermal power, $\mathrm{P}_{\mathrm{TH}}(\mathrm{MWt})$

Mass utilization, $\mathrm{M}_{\mathrm{FPC}} / \mathrm{P}_{\mathrm{TH}}$ (tonne/MWt)

FPC power density, $\mathrm{P}_{\mathrm{TH}} / \mathrm{V}_{\mathrm{FPC}}\left(\mathrm{MWt} / \mathrm{m}^{3}\right)$

FPC dimensions

- Spheromak radius, R(m)

- Spheromak hejght, L(m)

- Spheromak volume, $\pi R^{2} L\left(m^{3}\right)$

- First-wall radius, $r_{w}$

- EFC thickness, $\delta_{c}(m)$

- Inner electrode radius, $r_{g i}(m)$

- Outer electrode radius, $r_{\text {go }}(m)$

- Electrode length, $\ell_{g}(\mathrm{~m})$

- System radius, $r_{S}(m)$

- Mass (tonne)

Plasma Q-values, $Q_{p}=\mathrm{P}_{F} / \mathrm{P}_{\mathrm{EL}}$

Recirculating power fraction, $\varepsilon$

Unit direct cost, UDC $(\$ / \mathrm{kWe})$

Cost of electricity, COE(mills/kWe)
250. 500 .

17.4

18.7

19.8

19.8

973. 1,803 .

2605.

3416.

0.41

0.29

0.26

0.24

8.69

10.20

10.59

10.65

1.70

2.22

2.60

3.00

2.12

2.77

3.25

3.72

18.34

42.89

69.02

105.18

1.70

2.22

2.60

3.00

0.59

0.56

0.59

0.57

0.95

1.24

1.45

1.66

1.42

1.86

2.17

2.49

2.29

3.15

3.85

4.38

2.99

3.48

3.88

4.25 395. 524. 685.

806 .

37.

60.

76.

94.

0.27

0.21

0.10

0.16 $2,441.9 \quad 1,480.9 \quad 1,147.4$

977.7

109.4

67.0

52.2 
capacity, neutron wall loading, and mass power density can vary widely among concepts. These differences are illustrated in Fig. 5.1.-1, which gives the dependence of $\operatorname{COE}$ on $I_{W}, P_{E}$, and MPD for the CRFPR, CSR, and ATR/ST. A comparison of the COE versus net-electric-power economy-of-scale is shown in Fig. 5.1.-2 for both the CSR and a recent re-evaluation 92,93 of the RFP reactor that would use either superconducting (SC/RFP) or resistive (NC/RFP) EFCs. Shown also in Fig. 5.1.-2., are economy-of-scale curves for medium-experience (ME) and best-experience pressurized-water (fission) reactors (PWR), as well as fossil-fuelled power plants and a range of new-era modular fission reactor designs.94 Although subject to large uncertainty and extrapolation, the position of these compact, high-power-density fusion concepts relative to the bestexperience PWR, fossil fuel based on cheap coal or oil (10 \$/bbl $\approx 1.77$ \$/MBTU), and advanced fission systems is encouraging.

\subsection{Conclusions}

Table 5.2.-I summarizes key conclusions and provides a synoptic assessment derived from this preliminary scoping study. The spherical-like reactor geometry presents both advantages and disadvantages. Because of the higher ratio of plasma volume to surface, higher FPC mass power density occurs for a lower FPC power density, but the combination of high power density and high total power can be achieved only for higher first-wall neutron loadings. Equilibrium in a low-aspect-ratio, spherical-like geometry also requires large plasma currents, but both the plasma current density (and ohmic heating rates) as well as stored energy remain low.

The physics issues and the impact on reactor performance can be divided between the spheromak and the gun/electrode divertor. The sustained spheromak may require a constant $\mu=\mu_{0} \vec{j} \cdot \vec{B} / B^{2}$ profile, inferring that large Taylor currar.- must flow in the cold plasma edge. High edge-plasma temperatures are needed co minimize Joule losses ircurred therein, which in turn can impact the power consumed by the gun-electrode/divertor.

The divertor appears to be a key system in determining the viability of this concept as a steady-siate, stationary reactor. The exo-reactor location of the divertor gives greater design and maintenance flexibility to this high-heatflux unit. The impact of the divertor on blanket efficiency remains to be quantified. The gun scaling relationship [Eq. (3.3.-16)] represents a large uncertainty and totermines the fraction of poloidal flux diverted, wisich should 


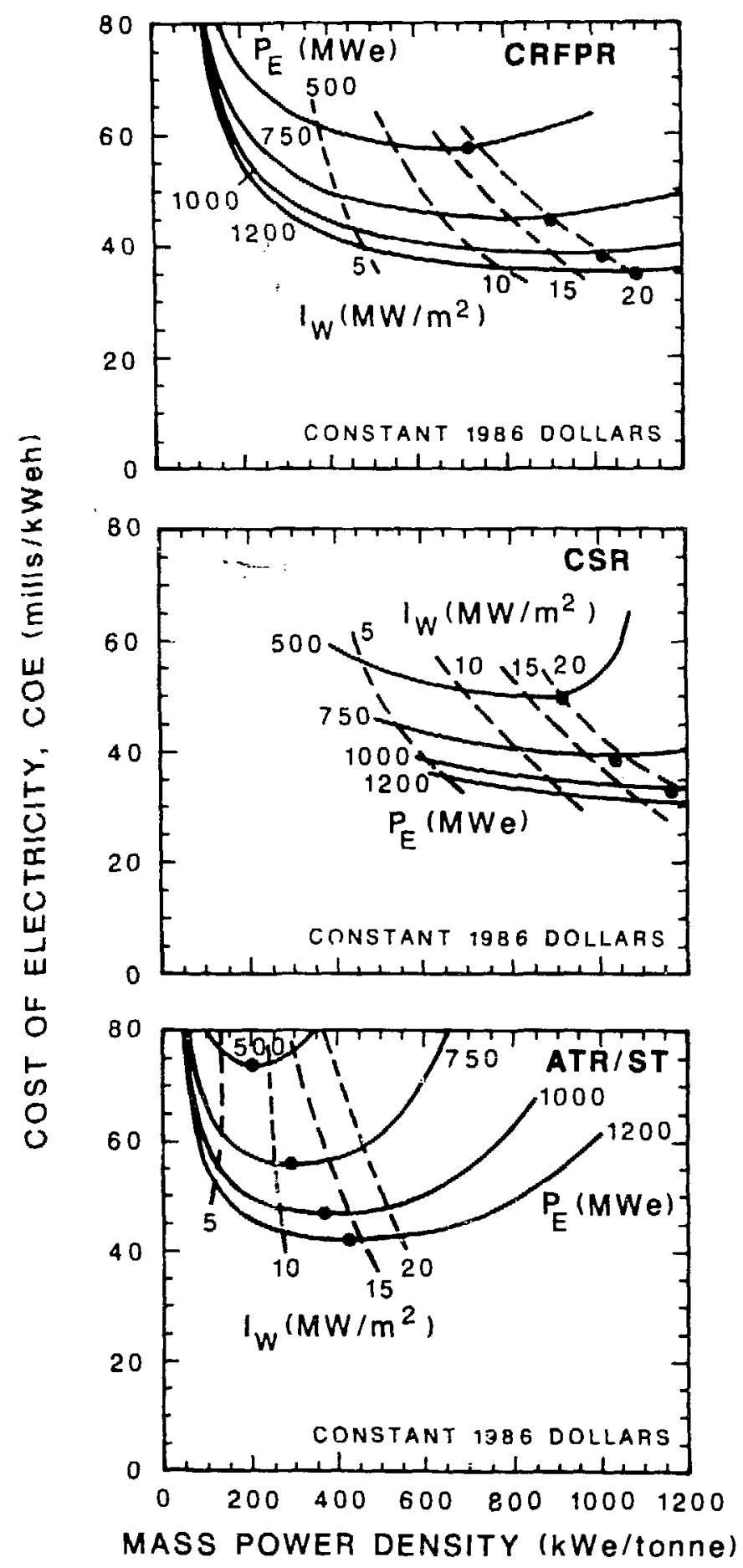

Fig. 5.1.-1. Dependence of $\mathrm{COE}$ on mass power density for a range of resistivecoil fusion concepts as a function of net-electric power and neutron wall loading. 


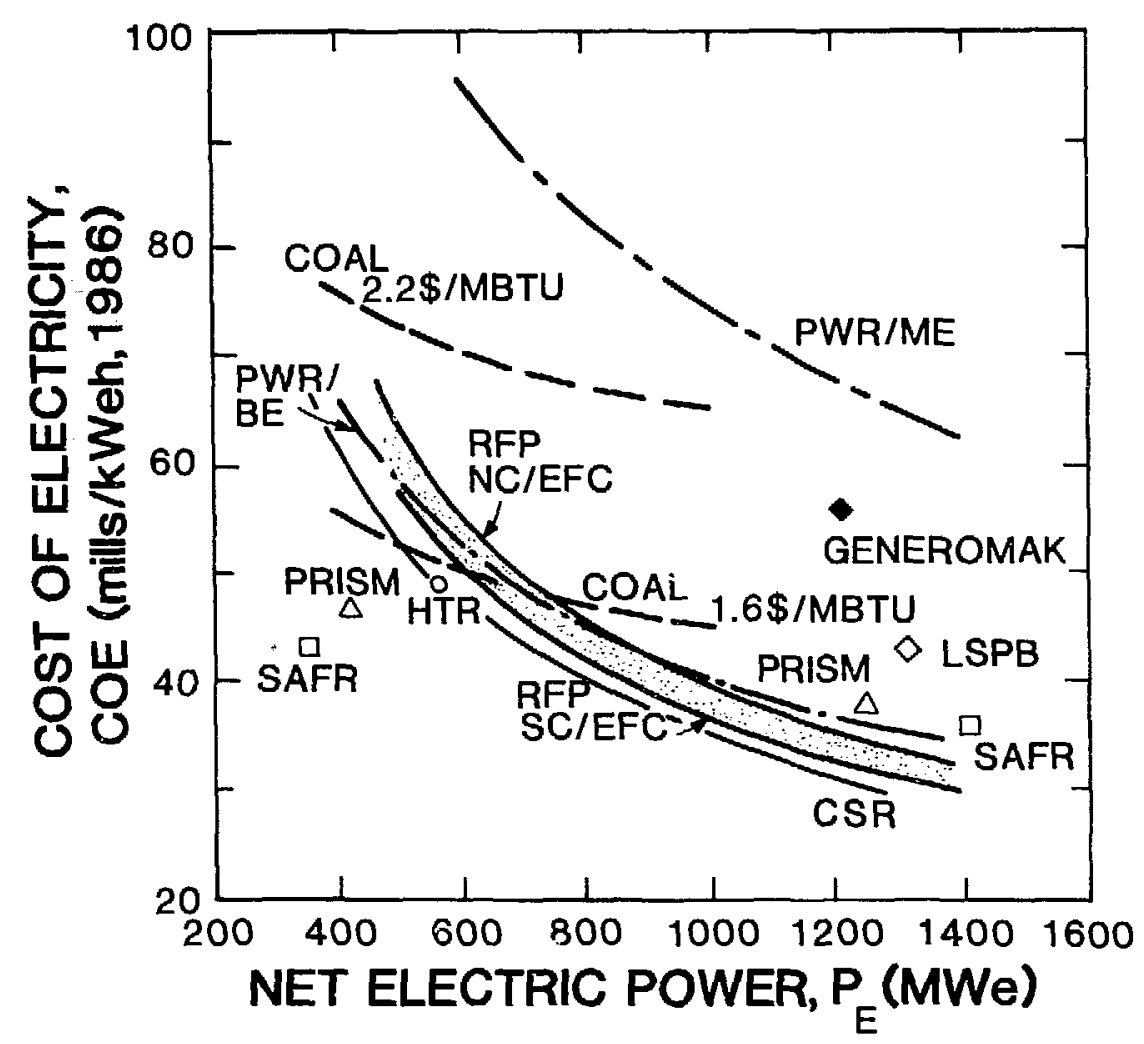

Fig. 5.1.-2. Economy-of-scale curves for the CSR (this study) compared to the reversed-field pinch (RFP), 25 medium- and best-experience pressurized-water reactors (PWR),94 fossil-fuelled (coal) plants, 94 and a range of new-era modular fission reactors ${ }^{24}$ (PRISM, SAFR, LSPB). Also shown is a projection made by a recent generic fission reactor study ${ }^{15}$ (GENEROMAK).

remain below -0.01 in order to minimize power requirements. The scaling of the flux term in Eq. (3.3.-16) with geometry, as parametrically modelled in this study by $f_{R}$, represents a particularly important issue, with $f_{R} \leq 0.5$ being required to access most designs of interest, and $f_{R} \geq 0.8$ leading to difficulties for all but the low-net-power, high-COE designs. Lastly, the plasma processes active in the transition region between the divertor and the spheromak involve magnetic helicity generation, transport, and absorption, and therefore, are characterized by related unknown processes.

The results presented herein appear to be promising from the viewpoint of simplified FPC, smaller nuclear system, and the possibility to shrink the nuclear envelope to an extent where fusion assumes an economy-of-scale that is more like fossil-fuel systems. In this case, smaller power plants would become 


\section{SYNOPTIC INTERIM ASSESSMENT}

- Spherical-like geometry infers

- lower FPC power density but with a lower FPC mass utilization (tonne/MWt) and higher mass power density (kWe/tonne)

- equilibrium requires higher total plasma current, but current density is low, as is stored energy $\left(\mathrm{L}_{\mathrm{p}} \mathrm{I}_{\phi}^{2} / 2\right)$

- Constant $\mu=\mu_{0} \vec{j} / \vec{B}$ plasma requires high current density at the plasma edge unlike the nodified Bessel-function models that describe RFPs

- high-temperature closed-field edge-plasma desirable (compatible with divertor operation)

- depending on helicity-injection efficiency and edge-plasma temperature, electrode/divertor power consumption can exceed plasma Joule losses, but both generally have small impact on overall power balance

- one-dimensional effects on efficiency of ohmic heating to ignition remain to be better understood and quantified

- Electrode/divertor system is a key to steady-state operation

- possible to reduce considerably the first-wall heat load

- exo-reactor position gives design flexibility to the high-heat-flux electrode/divertor system

- size dictated by heat load, determines blanket coverage (heat leakage, tritium-breeding ratio, etc.)

- combined current-drive (low-current, low-voltage), high-heat-flux, and ash/impurity-control functions; requires further understanding

- diversion of small fraction of poloidal flux $(\leq 1 \%)$ crucial to energy balance

- low arc voltage drop is important to energy balance

- uniformity of heat load and current density at electrode/divertor surface not known, but could strongly impact gun/electrode size

- Taylor-like plasma transition between spheromak and electrode/divertor system needs better characterization

- scaling of electrode/divertor current, flux, and voltage crucial to reactor, large extrapolation from present-day experiment, but a reduction in the flux scaling term by a factor of -2 most desirable for reactor, indicating need for geometry scaling results

- Low-field EFCs required

- efficient resistive coils

- reduced blanket/shield thicknesses (and total coil mass) possible

- low EFC fields (2.5-3.0 T) with high plasma fields (5-6 T) allow use of self-cooled liquid-metal high-power-density blankets

- low mass (400-700 tonne) eases maintenance and lowers FPC cost (RPE/TDC $\leq 0.3$ similar to fission power)

- Minimal COE penalty for lower FPC power density and neutron first-wall loading for same net power, but usual economy-of-scale penalties exist for lower total power output

- larger spheromaks than for RFP result

- may be possible to put CSR system on a fossil-plant-like economy-of-scale if nuclear envelope can be reduced (e.g., intermediate heat exchanger as steam generator coupled directly to small FPC) 
more attractive economically, multiplexing a number of small low-cost FPCs to drive a large total plant may be possible, and off-site factory construction of a greater part of the nuclear system may be possible. Lastly, "single-piece" maintenance of the FPC is also possible, opening the following innovative options and merits:

- Factory fabrication of almost fully operating unit

- Full operational, non-nuclear testing

- Minimize making and breaking connections in the nuclear environment

- Shortened schedule maintenance period

- reduced replacement time

- reduced restart period with increased confidence

- More standard or rapid recovery frcm unscheduled events

- Increased plant availability

- Accommodate fusion core improvement throughout the plant life

In summary, the CSR is one of a number of options and opportunities for significant improvement in the prospects for commercial fusion power based on the principal tokamak as well as othei concepts. The inter-relationship among these options (RFPs, spheromaks, FPCs, advanced tokamaks) is becoming clearer as physics understanding develops. One important direction for significant improvement is towards systems that assume more of the task of plasma confinement, heating, and sustainment through self-generated fields rather than by imposing these functions exclusively on complex and costly engineering systems that surround a low-power-density plasma. Systems that are dominated by poloidal fiels offer unique promise to reduce coil and, hence, FPC size, and to some degree may include tokamak varjants. Although the tokamak physics database is better developed than that for PFD systems, the degree to which these advanced tokamaks must be extrapolated from that database is not unlike that for the other approaches. Recent advances in these other concepts have been impressive, and the promise is great for development paths that alter considerably the previously assumed trend of ever-escalating device size and cost. A less costly but bolder and more flexible development patii to commercial fusion is anticipated for both these PFD systems as well as appropriately tailored variants of the tokamak. 
More detailed analyses of physics and technology constraints and the associated tradeoffs related to development cost and $t$ ime, end-product operational and cost issues, and general safety and resource concerns are required to define both the attractiveness and competitiveness of fusion power. Hence, in addition to stressing increased MPD and reduced COE; as was done in this study, the pursuit of improved fusion reactors to varying degrees must:

- Consider the potential for reduced total power output and associated capital investment, with the possibility of multiplexing a number of smaller FPCs to drive a larger total site electrical capacity.

- Emphasize and/or enhance passive safety (aga'ust a loss of coolant) through inherent FPC design characteristics, while maintaining an MPD of economic interest.

- Stress long-pulsed or steady-state plasma operation while addressing related issues of plasma current drive, heating, fueling, and inpurity/ash control.

- Simplify the FPC design in terms of reduced fields, stresses, and stored (magnetic) energy while using advanced materials and/or fabrication techniques only where clear-cut advantages are perceived.

- Maintain a high overall plant efficiency by utilizing direct energy conversicn (when possible), high coolant fluid temperatures, and minimum power recirculated to the FPC and associated support systems (i.e., coils, current drive, plasma heaters, coolant pumps).

- Emphasize physically small modular FPCs that assure a flexible develcpment path and ultimately factory (off-site) fabrication, full non-nuclear FPC pre-testing, and single- or few-piece FPC maintenance and repair.

The direction for improved fusion systems is multi-faceted, with increased MPD being one of a number of important approaches. The progress represented on the design evaluation depicted in Table 1.1.-I, as well as the developing options shown on Table 1.1.-III combine to indicate that fusion is on the right track, ultimately leading to an economic and environmentally attractive source of long-term energy.

\section{ACKNOHLEDGMENTS}

The authors express their gratitude to Tom Jarboe and Chris Earnes of Los Alamos for many long enlightening discussions of experimental and theoretical spheromak results. 


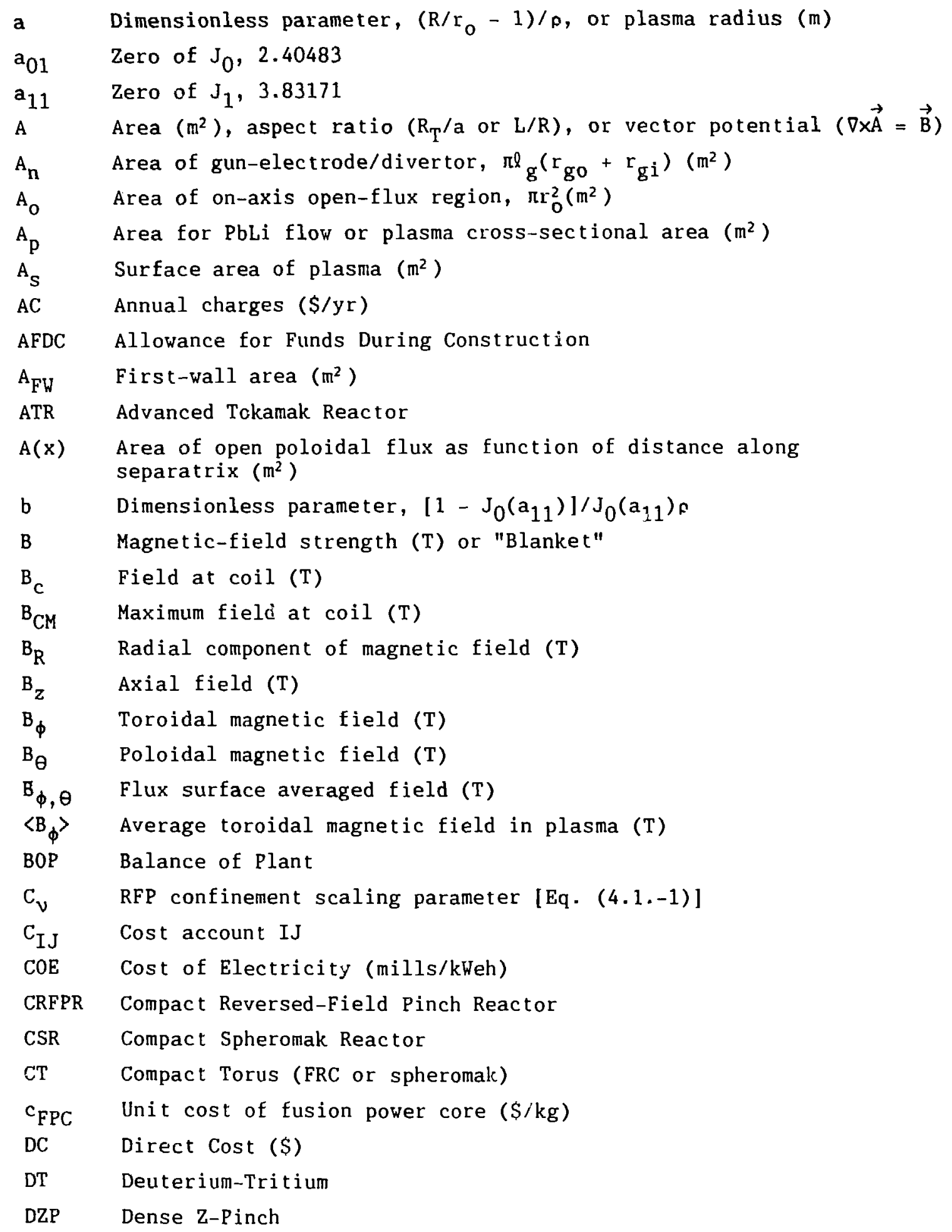




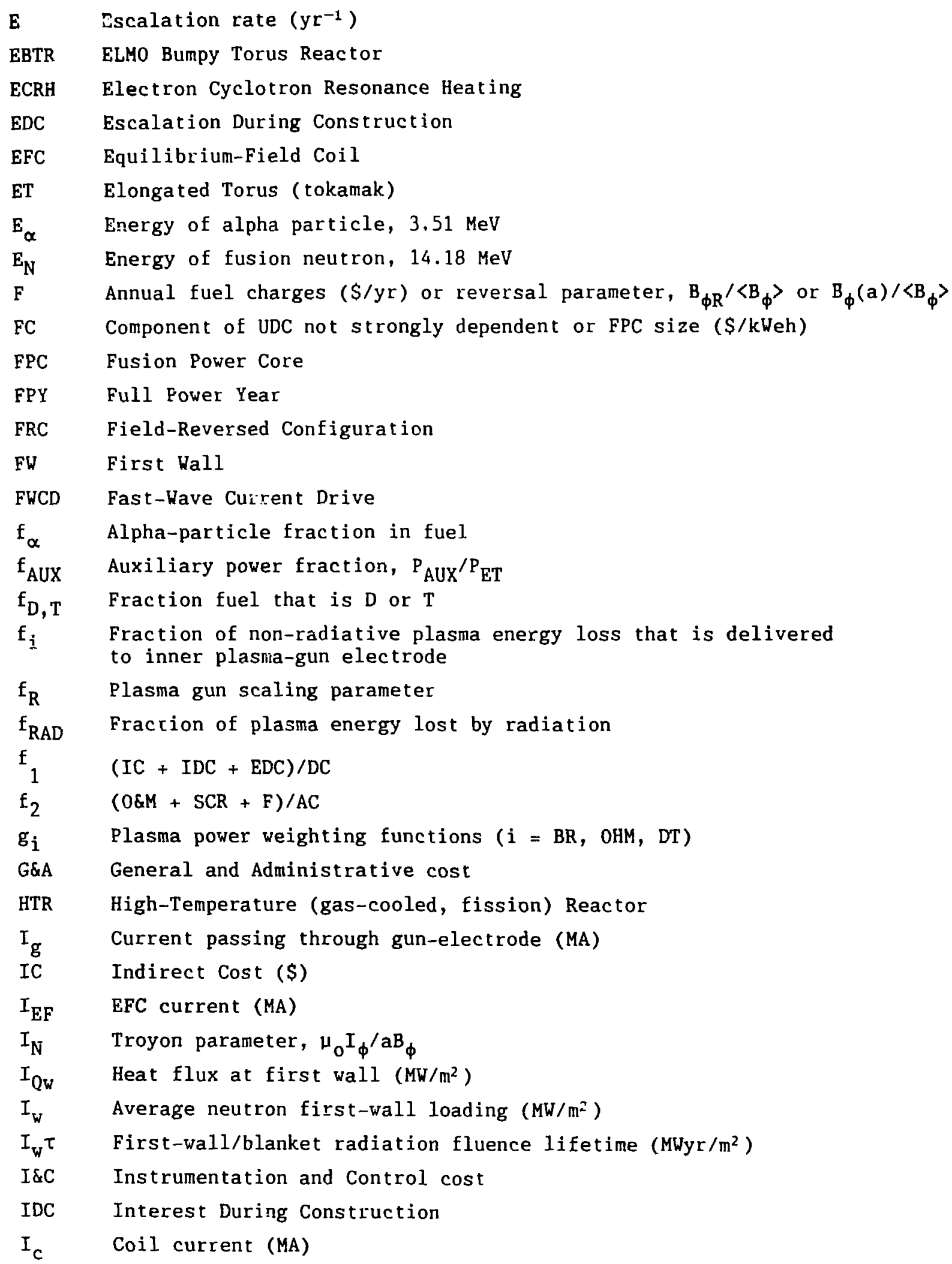




\begin{tabular}{|c|c|}
\hline $\mathrm{I}_{\boldsymbol{\phi}}$ & Plasma toroidal current (MA) \\
\hline $\mathrm{I}_{\theta}$ & Plasma poloidal current (MA) \\
\hline j & Current density $\left(\mathrm{MA} / \mathrm{m}^{2}\right)$ \\
\hline$j_{c}$ & Coil current density $\left(M A / m^{i}\right)$ \\
\hline$j_{\theta}$ & Plasma poloidal current density $\left(M \Lambda / m^{2}\right)$ \\
\hline $\mathbf{j}_{\phi}$ & Plasma toroidal current density $\left(\mathrm{MA} / \mathrm{m}^{2}\right)$ \\
\hline $\mathrm{k}_{\mathrm{B}}$ & Boltzmann constant $\left[1.602(10)^{-16} \mathrm{~J} / \mathrm{keV}\right]$ \\
\hline $\begin{array}{l}J_{0,1} \\
k\end{array}$ & $\begin{array}{l}\text { Zeroth- and first-order Bessel functions of the first kind } \\
\left(k_{x}^{2}+k_{z}^{2}\right)^{1 / 2}\end{array}$ \\
\hline K & Magnetic helicity $\left(\mathrm{Wb}^{2}\right)$ \\
\hline$k_{r}$ & Radial wave number, $a_{11} / R\left(m^{-1}\right)$ \\
\hline$k_{z}$ & Axial wave number, $\pi / L$ \\
\hline ! & Poloidal mode number, divertor chamber length \\
\hline $\lg$ & Length of gun-electrode/divertor $(\mathrm{m})$ \\
\hline${ }^{l} \mathrm{~L}$ & Length of PbLi coolant header to inner electrode (m) \\
\hline $\ln \Lambda$ & Coulomb logarithm \\
\hline $\mathrm{L}$ & Height of spheromak (m) \\
\hline LSPB & Large-Scale Power Breeder (fission) \\
\hline $\mathrm{L}_{\mathrm{p}}$ & Plasma inductance $(\mathrm{H})$ \\
\hline $\mathrm{L}_{E F}$ & EFC inductance ( $\mathrm{H})$ \\
\hline LWR & Light-Water Reactor \\
\hline $\mathrm{m}$ & Poloidal mode number \\
\hline $\mathrm{m}_{\mathrm{i}, \mathrm{e}}$ & Ion, electron mass $(\mathrm{kg})$ \\
\hline MFE & Magnetic Fusion Energy \\
\hline${ }_{E F, P}$ & Plasma/EFC mutual inductance $(\mathrm{H})$ \\
\hline MU & FPC Mass Utilization (tonne/MWt) \\
\hline${ }^{\mathrm{EFC}}$ & Mass of EFC (tonne) \\
\hline $\mathrm{M}_{\mathrm{N}}$ & FW/B/S neutron energy multiplication \\
\hline $\mathrm{M}_{\mathrm{N}} \mathrm{P}_{\mathrm{N}}$ & Recoverable nuclear heating (MW) \\
\hline${ }^{M}$ FPC & FPC mass (tonne) \\
\hline MHD & Magne tohydrodynamic \\
\hline MPD & Mass Power Density (kWe/tonne) \\
\hline MSR & Modular Stellarator Reactor \\
\hline $\mathbf{n}$ & Toroidal mode number, scrapeoff or average plasma density $\left(\mathrm{m}^{-3}\right)$ \\
\hline $\mathrm{n}_{\mathrm{i}}$ & Average plasma ion density $\left(\mathrm{m}^{-3}\right)$ \\
\hline $\mathrm{n}_{\mathrm{o}}$ & Separatrix density or initial or final density $\left(\mathrm{m}^{-3}\right)$ \\
\hline
\end{tabular}




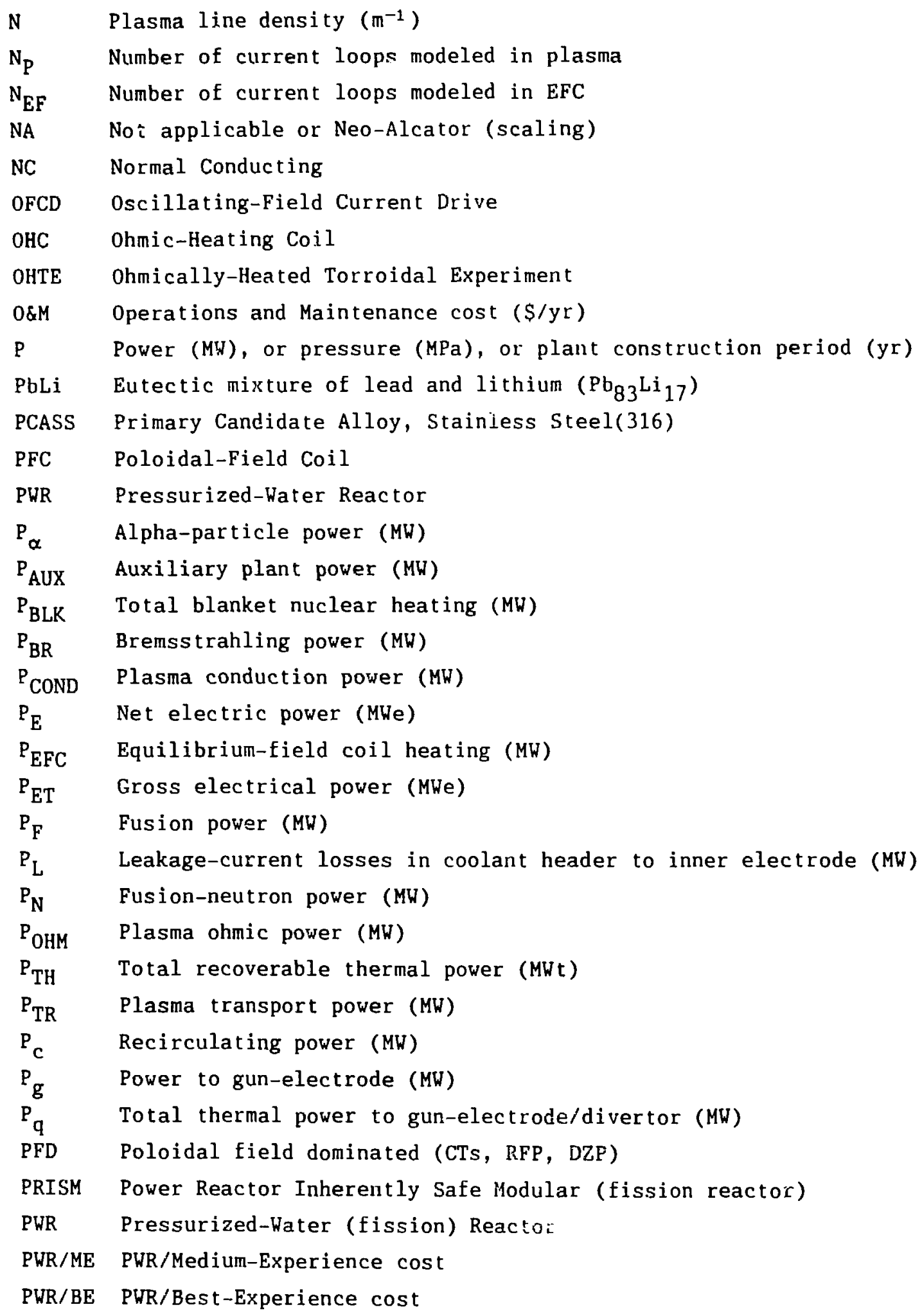




\begin{tabular}{|c|c|}
\hline $\mathrm{P}$ & Plasma pressure $(\mathrm{Pa})$ \\
\hline $\mathrm{P}_{\mathrm{f}}$ & Plant availability factor \\
\hline $\mathrm{q}$ & Plasma safety factor, $\left(B_{\phi} / B_{\theta}\right) /\left(r R_{T}\right)$ \\
\hline $\mathrm{q}_{\mathrm{a}}$ & Plasma safety factor at plasma edge \\
\hline $\mathrm{q}_{\mathrm{s}}$ & Surface heat flux $\left(\mathrm{MW} / \mathrm{m}^{2}\right)$ \\
\hline$q_{D I V}$ & Divertor channel heat flux $\left(\mathrm{MW} / \mathrm{m}^{2}\right)$ \\
\hline$q_{w}$ & First-wall heat flux $\left(\mathrm{MW} / \mathrm{m}^{2}\right)$ \\
\hline$Q_{E}$ & Engineering 0 -value, $\mathrm{P}_{\mathrm{ET}} / \mathrm{P}_{\mathrm{C}}=1 / \varepsilon$ \\
\hline$Q_{p}$ & Plasma 0 -value, $\mathrm{P}_{\mathrm{f}} / \mathrm{P}_{\Omega}$ \\
\hline$Q(x)$ & $F(x), A(x)$ Separatrix temperature-profile functions [Eq. (A-9)] \\
\hline $\mathrm{r}$ & Minor radius $(m)$ \\
\hline$r_{g i}$ & Inner electrode radius (m) \\
\hline $\mathrm{r}_{\text {go }}$ & Outer electrode radius (m) \\
\hline$r_{0}$ & Minor radius of inner separatrix (m) \\
\hline$r_{p}$ & Circularized plasma minor radius $(\mathrm{m})$ \\
\hline$r_{w}$ & First-wall minor radius (m) \\
\hline $\mathrm{R}$ & Major radius of separatrix $(\mathrm{m})$ \\
\hline RPE & Reactor Plant Equipment (Account 22.) \\
\hline$R_{p}$ & Plasma resistance (ohm) \\
\hline $\mathrm{R}_{\mathrm{T}}$ & Major toroidal radius of plasma (m) \\
\hline $\mathrm{RF}$ & Radio Frequency \\
\hline RFP & Reversed-Field Pinch \\
\hline$R \& D$ & Research and Development \\
\hline RPE & Reactor Plant Equipment \\
\hline SAFR & Sodium Advanced Fast (fission) Reactor \\
\hline SC & Superconducting \\
\hline $\mathrm{SCR}$ & Spare Component Replacement cost $(\$ / y r)$ \\
\hline SI & International System of Units (mks) \\
\hline SSR & Second Stability Region \\
\hline ST & Spherical Torus (tokamak) \\
\hline$t$ & Time (s) \\
\hline $\mathbf{T}$ & Temperature of coolant or average plasma $\left({ }^{\circ}, \mathrm{K}, \mathrm{keV}\right)$ \\
\hline $\mathrm{T}_{\mathrm{E}}$ & Edge-plasma temperature (kev) \\
\hline $\mathrm{T}_{\mathrm{i}, \mathrm{e}}$ & Ion, electron temperature $(\mathrm{keV})$ \\
\hline $\mathrm{T}_{\mathrm{o}}$ & Peak separatrix temperature $(\mathrm{keV})$ \\
\hline $\mathrm{T}_{\mathrm{d}}$ & Surface electrode/gun temperature (eV) \\
\hline
\end{tabular}




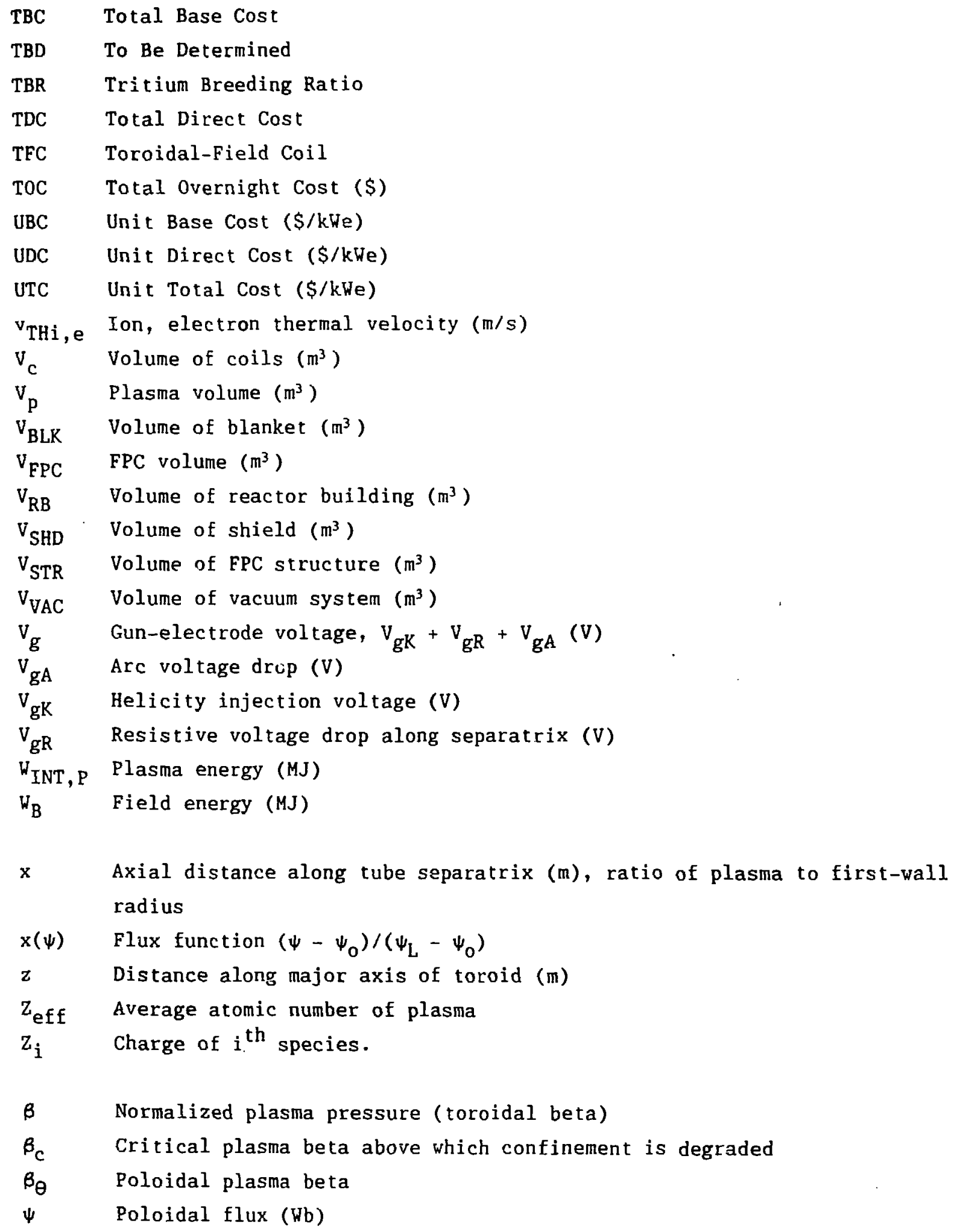




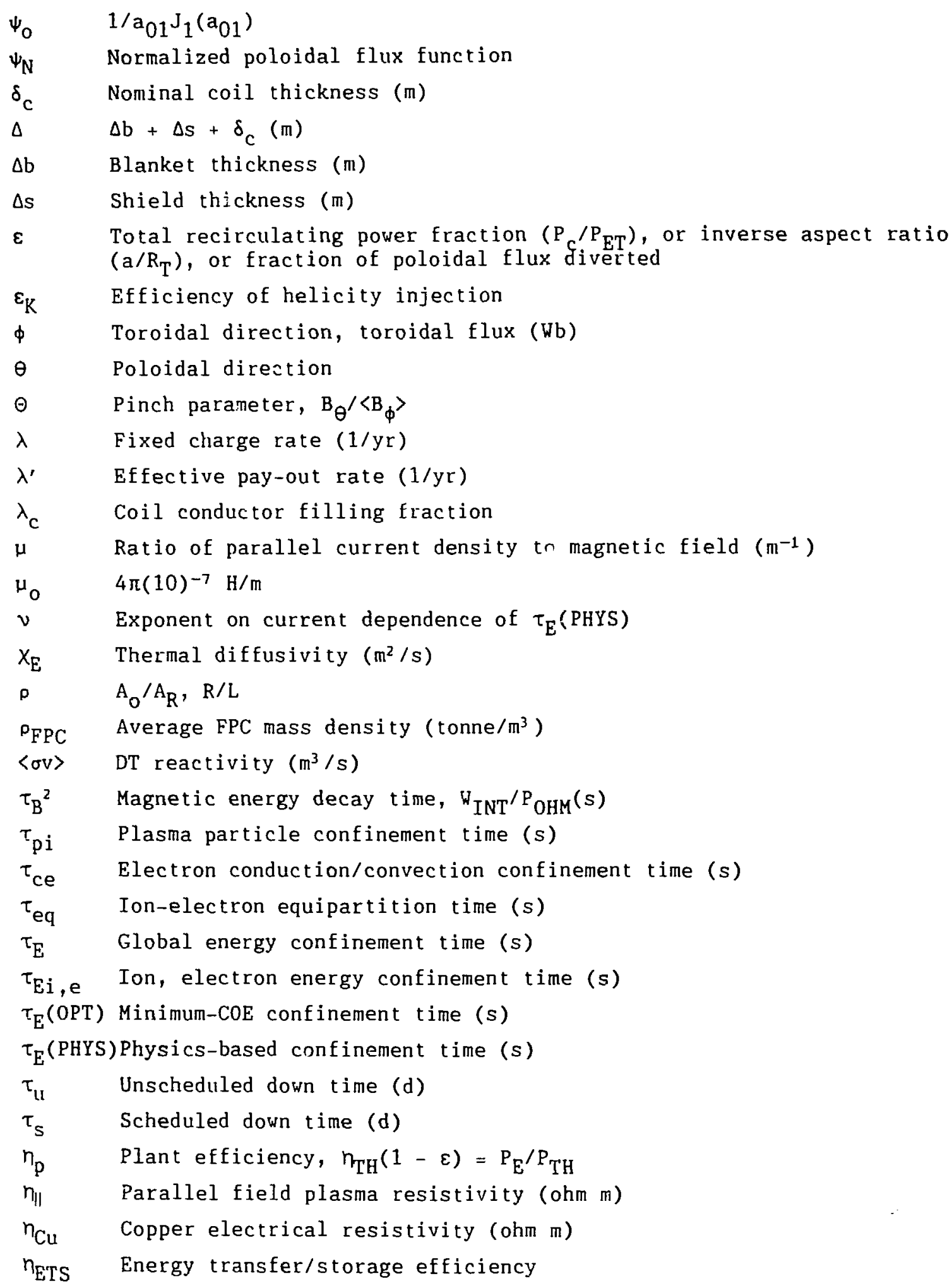


1. B. Badger, M. A. Abdou, R. W. Boom, R. G. Brown, T. E. Chang, R. W. Conn, et al., "UWMAK-I, A Wisconsin Toroidal Fusion Reactor Design," University of Wisconsin report UWFDM-68 (March 1974).

2. R. G. Mills, (Ed.), "A Fusion Power Plant," Princeton Plasma Physics Laboratory report MATT-1050 (August 1974).

3. F. F. Casali (Ed.), "FINTOR 1, a Minimum Size Tokamak Experimental Reactor," Euratom Ispra (October 1976).

4. C. C. Baker, M. A. Abdou, R. M. Arons, A. E. Bolon, C. D. Boley, J. N. Brooks, et al., "STARFIRE - A Commercial Tokamak Fusion Power Plant Study," Argonne National Laboratory report ANL/FPP-B0-1 (September 1980).

5. A. A. Hollis, "An Analysis of the Estimated Capital Cost of a Fusion Reactor," URAEA Harwell report AERE-R9933 (June 1981).

6. R. Hancox, R. A. Krakowski, and W. R. Spears, "The Reversed-Field Pinch Reactor," Nucl. Eng. and Design 63(2), 251 (1981).

7. R. L. Miller, C. G. Bathke, R. A. Krakowski, F. M. Heck, L. Green, J. S. Karbowski, et al., "The Modular Stellarator Reactor: A Fusion Power Plant," Los Alamos National Laboratory report LA-9737-MS (July 1983).

8. B. Badger, I. N. Sviatoslavsky, S. W. Vansciver, G. L. Kulcinski, G. A. Emmert, D. T. Anderson, et al., "UWTOR-M: A Conceptual Modular Stellarator Power Reactor," University of Wisconsin report UWFDM-550 (October 1982).

9. C. G. Bathke, D. J. Dudziak, R. A. Krakowski, W. R. Ard, A. A. Bowers, J. W. Davis, et al., "ELMO Bumpy Torus Reactor and Power Plant Conceptual Design Study," Los Alamos National Laboratory report LA-8882-MS (August $1981)$.

10. B. G. Logan, C. D. Henning, G. A. Carlson, R. W. Werner, D. E. Baldwin, W. L. Barr, et al., "MARS: Mirror Advanced Reactor Study," Lawrence Livermore National Laboratory report UCRL-53480 (July 1984).

11. R. Carruthers, "Criteria for the Assessment of Reactor Potential," Unconventional Approaches to Fusion, B. Brunelli and G. G. Leatton (eds.), Pp. 39-45, Plenum Press, NY (1982).

12. L. M. Lidsky, "The Trouble With Fusion," Technol. Rev. 32 (October 1983).

13. D. Pfirsch and K. H. Schmitter, "Some Critical Observations on the Prospects of Fusion Power," 4 th Inter. Conf. on Energy Options--The Role of Alternatives in the World Energy Scene, London, April 3-6, 1984 (IEE Conf. Publ. No. 233).

14. R. A. Krakowski, R. L. Hagenson, and R. L. Miller, "Small Fusion Reactors: Problems, Promise, and Pathways," Proc. 13th Symp. on Fusion Technology (SOFT), Varese, Italy, 1, 45 (September 24-28, 1984). 
15. J. Sheffield, R. A. Dory, S. M. Cohn, J. G. Delene, L. Parsly, D. E. T.F. Ashby, and W. T. Reirsen, "Cost Assessment of a Generic Magnetic Fusion Reactor," Fus. Technol. 9, 199 (1986).

16. R. A. Krakowski, "Fusion Concepts with Improved Prospects for Reactor," Proc. 20th Intersoc. Eil. Conv. Eng. Conf. (IECEC), 3, 3.6, Miami Beach, Florida (August 18-23, 1985).

17. C. C. Baker, "New Directions in Tokamak Reactors," Fus. Technol., $\underline{8}(1)$, 707 (1985).

18. R. K. Linford, "New Directions in Fusion Machine: Report on the MFAC Panel X High-Power-Density Options," Proc. 11 th Symp. on Fusion Eng., Austin, TX (November 18-22, 1985).

19. R. Buende, "Cost Sensitivity, Analysis of Possible Fusion Power Plants," Atomkernenergie (ATKE) 30, 183 (1977).

20. "Guide for Economic Evaluation of Nuclear Reactor Designs," NIS Corporation report NUS-531 (January 1969).

21. S. C. Schulte, W. E. Bickford, C. E. Willinghan, S. K. Ghose, and M. G. Walker, "Fusion Reactor Design Studies--Standard Unit Costs and Cost Scaling Rules," Pacific Northwest Laboratory report PNL-4987 (September 1979).

22. W. R. Hamilton, D. C. Keeton, and S. L. Thompson, "Cost Accounting Systems for Fusion Studies," Gak Ridge National Laboratory report ORNL/FEDC-85/7 (December 1985).

23. J. H. Crowley and R. E. Allan, "Phase VII Update (1984) Report for the Energy Economic Data Base Program: EEDB-VII," Department of Energy report DOE/NE-0051/2 (August 1985).

24. R. L. Miller, R. A. Krakowski, C. G. Bathke, C. Copenhaver, A. Engelhardt, T. J. Seed, R. M. Zubrin, and N. M. Schnurr, "Advanced Tokamak Reactors Based on the Spherical Torus (ATR/ST): Preliminary Design Considerations," Los Alamos National Laboratory report LA-10740-MS (May 1986).

25. R. A. Krakowski, R. L. Hagenson, N. M. Schnurr, C. Copenhaver, C. G. Bathke, and R. L. Miller, "Fusion-Power-Core Integration Study for the Compact Reversed-Field Pinch Reactor (CRFPR): A Follow-On Study," Nucl. Eng. and Design $\underline{4}, 75$ (1986).

26. R. L. Hagenson, R. A. Krakowski, C. G. Bathke, R. L. Miller, M. J. Embrechts, N. M. Schnurr, "Compact Reversed-Field Pinch Reactors (CRFPR): Preliminary Engineering Considerations," Los Alamos National Laboratory report LA-10200-MS (August 1984).

27. R. L. Hagenson and R. A. Krakowski, "Steady-State Spheromak Reactor Studies," Fus. Technol. $\underline{8}$ (1), 1606 (1985).

28. Monthly Energy Review, Energy Information Administration (August 1985). 
29. B. G. Logan, "A Rationale for Fusion Economics Based on Inherent Safety," J. Fus. En. 4(4), 245 (1985).

30. R. J. Sutherland, A. Ford, S. V. Jackson, C. A. Mangeng, R. W. Hardie, R. E. Malenfant, "The Future Market for Electric Generating Capacity: Technical Documentation," Los Alamos National Laboratory report LA-10285-MS (March 1985).

31. R. K. Lester, "Rethinking Nuclear Power," Sci. Amer., 254(3), 31 (1986).

32. Y-K. M. Peng, "Spherical Torus Compact Fusion at Low Field," Oak Ridge National Laboratory report ORNL/FEPC-84/7 (February 1985).

33. S. C. Jardin, "Beta Limits for Large Noncircular Tokamaks and the Implications for Reactor Design," Proc. 10th Inter. Conf. on Plas. Phys. and Cont. Nucl. Fus. Res., paper IAEA-CN-44/A-IV-3, London (1984).

34. R. C. Grimm, "MHD Stability of Bean-Shaped Tokamaks," Princeton Plasma Physics Laboratory report PPPL-2090 (March 1984).

35. J. L. Johnson, "Recent Developments in Stellarator Physics," Nucl. Technol./ Fusion $4(2), 1275$ (1983).

36. R. L. Miller, "Recent Progress in Stellarator Reactor Conceptual Design," Fus. Technol. $\underline{8}(1), 1581$ (1985).

37. N. A. Uckan, "ELMO Bumpy Square," Oak Ridge National Laboratory report ORNL/TM-9110 (October 1984).

38. R. L. Hagenson, A. S. Tai, R. A. Krakowski, and R. W. Moses, "The Dense Z-Pinch (DZP) as a Fusion Power Reactor: Preliminary Scaling Calculations and System Energy Balance," Nucl. Fus. 21(11), 1351 (1981).

39. R. L. Hagenson and R. A. Krakowski, "A Compact-Toroid Fusion Reactor Based on the Field-Reversed Theta Pinch," Los Alamos National Laboratory report LA-8758-MS (March 1981).

40. L. J. Perkins, B. G. Logan, R. B. Campbell, R. S. Devoto, D. T. Blackfield, and B. H. Johnson, "Plasma Engineering for MINIMARS: A Small Commercial Tandem Mirror Reactor with 0ctopole Plugs," Fus. Technol. $\underline{8}(1), 685$ (1985).

41. R. S. Massey, R. G. Watt, P. G. Weber, G. A. Wurden, D. A. Baker, C. J. Buchenauer, et al., "Status of the ZT-40M RFP Experimental Program," Fus. Technol. $\underline{8}(1), 1571$ (1985).

42. H. Bodin, R. A. Krakowski, S. Ortolani, "The Reversed-Field Pinch: From Experiment to Reactor," Fus. Technol. 10(3), 307 (1986).

43. J. B. Taylor, "Relaxation of Toroidal Plasma and Generation of Reversed Magnetic Field," Phys. Letts. 33, 1139 (1974). 
44. J. B. Taylor, "Relaxation and Magnetic Reconnection in Plasma," Revs. Mod. Phys., 58(741) (1986).

45. T. R. Jarboe, "Spheromak Studies on CTX," Proc. 10th Inter. Conf. on Plas. Phys. and Cont. Nucl. Fus. Res., paper CN-44/DIII-1, London (September 12-19, 1984).

46. W. C. Turner, "Prcduction of a Field-Reversed Plasma With a Magnetized CoAxial Gun," Phys. Rev. Lett. 52(1), 175 (1981).

47. G. C. Goldenbaum, "Formation of a Spheromak Plasma Configuration," Phys. Rev. Lett. 44(1), 393 (1980).

48. M. Yamada, "Quasistatic Formation of a Spheromak Plasma Configuration," Phys. Rev. Lett. $46(1), 188$ (1981).

49. M. Katsurai and M. Yamada, "Studies of Conceptual Spheromak Fusion Reactors," Nucl. Fus. 22(11), 1407 (1982).

50. K. Uo, A. Iiyoshi, T. Obiki, 0. Motojima, S. Morimoto, M. Wakatani, et al., "NBI Heating of Currentless Plasma in Heliotron E," Tenth Int. Con $\bar{f}$. on Plasma Physics and Cont. Nuc. Fus. Research, London, UK (September 1984 ), paper IAEA-CN-44/D-I-2.

51. G. Cattanei, D. Dorst, A. Elsner, V. Erckmann, G. Grieger, P. Grigull, et al., "Plasma Confinement and the Effect of Rotational Transform in the Wendelstein VII-A Stellarator," ibid., paper IAEA-CN-44/D-I-1.

52. M. Greenwald, D. Gwinn, S. Milora, J. Parker, P. Parker, S. Wolfe, et al., "Pellet Fueling Experiments in Alcater C," ibid., paper IAEA-CN-44/A-I-I-

53. A. Kitsunezake, M. Abe, T. Hirayama, K. Hoshino, A. Kameari, K. Kodama, "High Pressure Plasma with High Power NBI Heating in Doublet III," ibid., paper IAEA-CN-44/A-I-4.

54. M. Abdou, E. Bertolini, R. Hancox, W. J. Hogan, A. I. Kosterbo, G. L. Kulcinski, et al., "Fusion Reactor Design-IV," Nucl. Fus. (October 1986).

55. P. C. Efthimion, N. Bretz, M. Bell, M. Bitter, W. R. Blanchard, F. Boody, "Confinement Studies of Ohmically-Heated Plasmas in TFTR," ibid, paper IAEA-CN-44/A-I-2.

56. T. Tamano, W. D. Bard, T. N. Carlstrom, C. Chu, B. Curwen, R. K. Fisher, et al., "High Current, High Beta Toroidal Pinch Experiments in OHTE," ibid., paper IAEA-CN-44/D-II-1.

57. J. N. DiMarco and the ZT-40 Team, "Experimental and Theoretical Studies of the ZT-40M Reversed-Field Pirch," ibid., paper IAEA-CN-44/D-II-2.

58. T. R. Jarboe, C. H. Barner, I. Henins, H. W. Hoida, S. 0. Know, A. G. Sgro, et al., "Spheromak Studies on CTX," ibid., paper IAEA-CN-44/D-III-1. 
59. M. Yamada, R. Ellis, Jr., H. P. Furth, G. Hart, D. Jassby, A. Janos, et al., "Initial Results from S-1 Spheromak," ibid., paper IAEA-CN-44/D-III- $\overline{3}$.

60. R. E. Siemon, W. T. Armstrong, R. E. Chrien, P. L. Klinger, R. K. Linford, K. F. Mckenna, et al., "Theoretical Studies of Field-Reversed Configurations $\overline{(F R C s)}$ and Experimental studies of the FRC During Translation," ibid., paper IAEA-CN-44/D-III-2-1.

61. A. L. Hoffman, J. T. Slough, R. D. Milroy, D. G. Harding, ' and L. C. Steinhauer, "Lifetime Scaling of Field Reversed Configurations," ibid., paper IAEA-CN-44/D-IV-1.

62. "Janes Fighting Ships: 1986-87," Ianes Publisuing re. Itd , I tandon (1900).

63. United Engineers and Constructors, Inc., "1000-MWe Central Power Plants Investment Cost Study," A.S. AEC report WASH-1230 (June 1972).

64. A. C. Smith, Jr., Project Leader, "Conceptual Design of a Moving-Ring Reactor," 9 th Int. Conf. on Piasma Physics and Cont. Nuclear Fusion Research, Baltimore, MD (1982).

65. A. Mohri, Y. Fugi-ie, K. Ikuta, H. Momota, H. Naitou, Y. Nomuro, et al., "Conceptual Design of a Moving Ring Reactor, KARIN-I," Fus. Technol. 8(1), 1596 (1985).

66. A. M. M. Todd, R. E. 0lson, J. G. Gilligan, G. H. Miley, "The Spheromak Fusion Reactor," Proc. 15th Inter. Soc. Energy Conv. Eng. Conf., p. 2229, Seattle, WA (1980).

67. C. Pferish, R. Gruber, and F. Troyon, "Free-Boundary MHD Stability of Pressureless 0blate Spheromaks - Dependence on Aspect Ratio and Elongation," Nuclear Fusion 23(9), 1127-1134 (1983).

68. T. R. Jarboe, "Steady State Spheromak," 5th Symp. on Physics and Tech. of Compact Toroids, Bellevue, WA (November 1982).

69. W. Watanabe, private communication, Osaka University, Japan (1985).

70. A. Janos, "Steady-State Operation of Spheromaks by Inductive Techniques," Princeton Plasma Physics Laboratory report PPPL-2095 (April 1984).

71. M. K. Bevir and J. W. Gray, "Relaxation, Flux Consumption and Quasi Steady State Pinches," Proc. RFP Theory Workshop, Los Alamos, NM (April 28 - May 2, 1980).

72. K. F. Schoenberg, C. J. Buckenauer, R. S. Massey, J. G. Melton, R. W. Moses, Jr., R. A. Nebel, and J. A. Phillips, "F- $\theta$ Pumping and Field Modulation Experiments on a Reversed Field Finch Discharge," Phys. Fluids 27(3), 548 (1984).

73. M. K. Bevir, C. G. Gimblott, and G. Miller, "Quasi Steady State Current Drive by Plasma Relaxations," Proc. IAEA Technical Commitlee Meeting on Non-Inductive Current Drive in Tokamaks, Culham Laboratory, UK (April 18-21, 1983). 
74. C. W. Barnes, J. C. Fernandez, I. Henins, H. W. Hoida, T. R. Jarboe, S. 0. Knox, G. J. Marklin, K. F. McKenna, "Experimental Determination of the Conservation of Magnetic Helicity from the Balance Between Source and Spheromak," Phys. Fluids 29(10), 3415, (1986).

75. T. R. Jarboe, C. W. Barnes, D. A. Platts, and B. L. Wright, "A Kinked Z-pinch as the Helicity Source for Spheromak Generatjon and Sustainment," Comments on Plasma Phys. $\underline{9}(4), 161$ (1985).

76. C. Copenhaver, R. A. Krakowski, N. M. Schnurr, R. L. Miller, C. G. Bathke, R. L. Hagenson, "Compact Reversed-Field Pinch Reactors (CRFPR): Fusion-Power-Core Integration Study." Los Alamos National Laburatory report LA-1500-MS (August 1985).

77. W. C. Turner, G. C. Goldenbaum, E. H. A. Grannenian, J. H. Hammer, C. W. Hartman, D. S. Prono, and J. Taska," Investigations of the Magnetic Structure and the Decay of a Plasma-Gun-Generated Compact Torus," Phys. Fluids 26(7), 1965-1986 (1983).

78. A. Bondeson, G. Marklin, Z. G. An, H. H. Chen, Y. C. Lee, and C. S. Lin, "Tilting Instability of a Cylindrical Spheronak," Phys. Fluids 24(9), $1682-1688$ (1981).

79. J. M. Finn, W. M. Manheimer, and E. Ott, "Spheromak Tilting Instability in Cylindrical Geometry," Naval Research Laboratory report NRL-4316 (1980).

80. M. N. Rosenbluth and M. N. Bussac, "MHD Stability of Spheromak," Nucl. Fus. $19(4), 489-498$ (1979).

81. E. K. Maschke, "Exact Solutions of the MHD Equilibrium Equation for a Toroidal Plasma," Plas. Phys. 15, 535-541 (1973).

82. G. K. Morikawa, "Double-Toroidal Hydromagnetic-Equilibrium Configurations Within a Perfectly Conducting Sphere," Phys. Fluids 12(8), 1648-1651 (1969).

83. H. L. Berk, J. H. Hammer, and H. Weitzner, "Analytic Field-Reversed Equilibria," Phys. Fluids 24(9), 1758-1759 (1981).

84. C. W. Barnes, T. R. Jarboe, I. Henins, A. R. Sherwood, S. 0. Knox, R. Gribble, et al., "Spheromak Formation and Operation with Background Filling Gas and a Solid Flux Conserver in CTX," Nucl. Fus. 24, 267 (1985).

85. T. R. Jarboe, C. W. Barnes, I. Henins, H. W. Hoida, S. 0. Knox, R. K. Linford, and A. R. Sherwood, "The ohmic Heating of a Spheromak to $100 \mathrm{eV}, "$ Phys. Fluids 27(1), 13-15 (1984).

86. P. J. Harbour and J. G. Morgan, "Models and Codes for the Plasma Edge Region," Culham Laboratory report CLM-R234, UK (1982).

87. V. D. Shafranov, Review of Plasma Physics, Vol. 2 p. 103. Consultants Bureau, NY, (1966). 
88. J. M. Greene, J. L. Johnson, and K. E. Weiner, "Tokamak Equilibrium," Phys. Fluids 14, 671 (1971).

89. V. S. Mukhavatov and V. D. Shafranov, "Plasma Equilibrium in a Tokamak," Nucl. Fus. 11, 605 (1971).

90. W. R. Smythe, "Static and Dynamic Electricity," Third Edition, McGraw-Hill Book Company, NY, (1968).

91. R. A. Krakowski, R. L. Miller, and J. G. Delene, "Directions for Improved Fusion Reactors," IAEA Tech. Comm. and Workshop on Fusion Reactor Design and Technology, Yal ta, USSR (May 26 - June 6, 1986).

92. R. L. Miller, R. A. Krakowski, C. G. Bathke, K. A. Werley, and R. L. Hagenson, "Fusion Reactor Options and Alternatives for the RFP," 7 th ANS Top. Mtg. on the Tech. of Fusion Energy, Reno, NV (June 15-19, 1986) (submit ted to Fus. Technol.).

93. R. V. Conn, "The Reversed-Field Pinch as a Compact Fusion Reactor: A Program in Conceptual Reactor Design, Technology Assessment, and Development Pathway Analysis," UCLA report PPG-969 (April 1986).

94. D. B. Tauger (ed.), "Nuclear Options Viability Study: Volume III, Nuclear Discipline Topics," Oak Ridge National Laboratory report ORNL/TM-9780/3 (May 1986). 
APPENDIX A: Estimate of Heat Loss From a "Cylindrical" Spheromak

The normalized poloidal flux for a cylindrical spheromak of radius $R$ and height $\mathrm{L}$ is given by

$$
\psi_{N}(r, z)=\psi_{0}\left(k_{r} r\right) J_{1}\left(k_{r} r\right) \sin k_{z} z
$$

with $\psi_{0} \equiv 1 / a_{01} J_{1}\left(a_{01}\right), k_{r}=a_{11} / R, k_{z}=\pi / L$, and the zeros for the zeroth- and first-order Bessel functions are $a_{01}=2.4048$ and $a_{11}=3.8317$, respectively, $\left[\mathrm{J}_{0}\left(\mathrm{a}_{11}\right)=-0.4028, \mathrm{~J}_{1}\left(\mathrm{a}_{01}\right)=0.5192\right.$, and $\psi_{\mathrm{N}}\left(\left(\mathrm{a}_{01} / \mathrm{a}_{11}\right) \mathrm{R}, \mathrm{L} / 2\right)=1$ ai the magnetic axisl. If the fraction $\varepsilon$ of this flux is diverted to a co-axial electrode, then the function

$$
\varepsilon=\psi_{0}\left(k_{r} r\right) J_{1}\left(k_{r} r\right) \sin k_{z} z
$$

defines a surface across which any heat and particle flux transported will strike the plasma-gun electrode system.

Both the heat flow and temperature distribution in the region bounded by the "cylindromak" ( $(\mathrm{L}, \mathrm{R})$ and the surface given by Eq. ( $\mathrm{A}-2)$ must be estimated to understand key features of a spheromak reactor that is sustained in steady state by a co-axial piasma-gun/divertor system. Specifically, the power partition between inner and outer electrodes determines the size of the respective heattransfer surfaces; this power equals the alpha-particle heating and is substantial. The temperature distribution along the surface of diverted flux is also needed to estimate ohmic losses in this region, which because of the large currents can also be substantial; these latter losses have been estimated in Eq. $(3.4 \cdot-5)$.

A simplified geometry for the diverted flux surface is used in conjunction with a one-dimensional steady-state heat-transport calculation to estimate both the energy partition between inner and outer divertor electrodes and the temperature distribution along this region of divertor flux. Figure A-1 illustrates the cylindrical geometry and an exaggerated view of the diverted 
flux. The thickness of these diverted-flux regions at the midplain and at the magnetic axis is used to approximate the heat-transport geometry given on Fig. A-2. Specifically, since $J_{1}\left(k_{r} r\right) \simeq k_{r} r / 2$ for small values of $k_{r} r$, it follows from Eq. (A-2) that

$$
r_{0}^{2}=\frac{2 \varepsilon a_{01} \mathrm{~K}^{2}}{a_{11}^{2}} J_{1}\left(a_{01}\right)
$$

Also, $B_{z}(R, L / 2) \simeq B_{0} J_{0}\left(a_{11}\right)$, where $B_{0}=B_{z}(0, L / 2)$ is the peak field along the geometric axis at the midplane. Conservation of flux gives

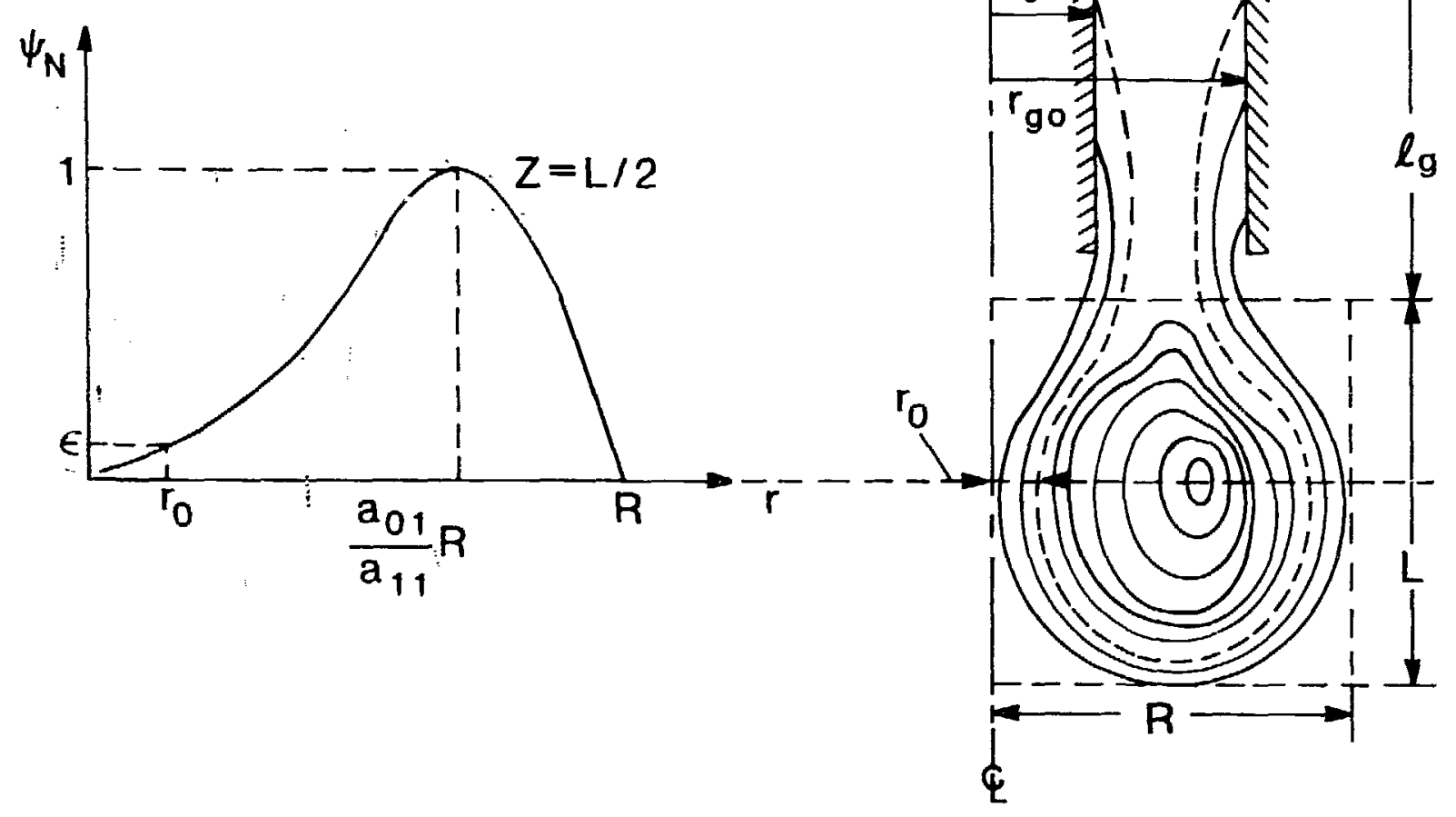

Fig. A-1. Geometry used to analyze the gun (electrode) properties, where the fraction of the poloidal flux, $\varepsilon$ is diverted inside the radius $r_{0}$ at the plasma surface. A cylindrical version shown in Fig. A-2 is used to estimate heat transport and Joule losses in the scrapeoff surrounding the spheromak. 


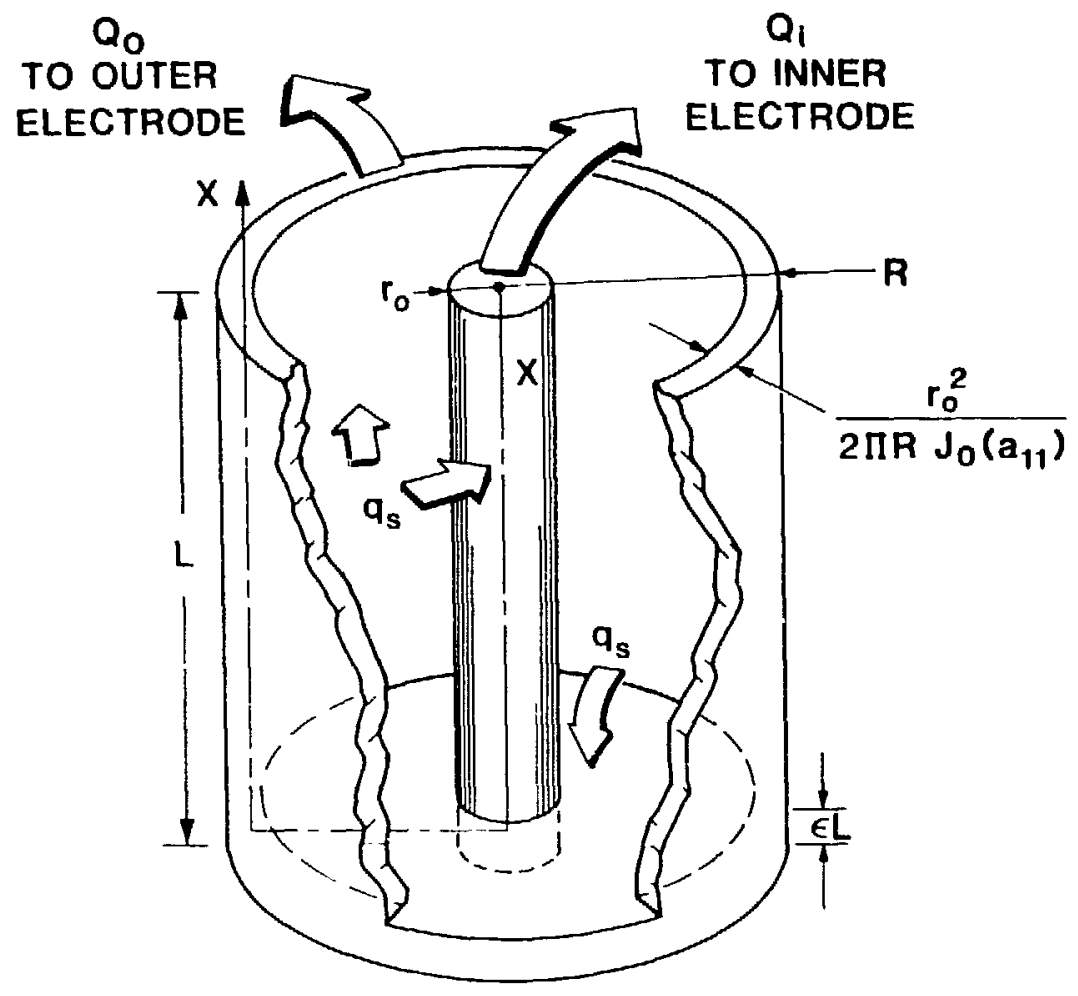

Fig. A-2. Cylindrical spheromak scrapeoff used to model heat transport from the spheromak to the electrodes.

$$
R-r_{0}^{\prime}=\frac{r_{0}^{2}}{2 R i J_{0}\left(a_{11}\right) \mid}
$$

which gives an area at the midplane and $r=R$ location that is available for heat transport to the plasma-gun electrode equal to $\pi r_{0}^{2} /\left|J_{0}\left(a_{11}\right)\right|$. Area variations in the z-directions are not taken into account by the model depicted in Fig. A-2. The conducting centerline cylinder of area $\pi r_{0}^{2}$ is connected to the thin outer cylindrical sheath of area $\pi r_{0}^{2} / J_{1}\left(a_{11}\right)$ by a bottom "plate" of thickness $\varepsilon \mathrm{L}$, which is the thickness of diverted flux evaluated at the magnetic axis $\left[\mathrm{r}=\left(\mathrm{a}_{01} / \mathrm{a}_{11}\right) \mathrm{R}, \quad z=0\right]$. The temperatures at $z=L$ and $\mathrm{r}=0$ or $\mathrm{R}$ are taken as constant and equal for the purposes of determining the inner/outer electrode power splits. 
The one-dimensional steady-state heat-transport equation given below is then solved for $T(x)$,

$$
\frac{\partial}{\partial x}\left[A(x) k \frac{d T}{d x}\right]=-q_{s}(x) P(x)
$$

where the $x$-axis originates at $(r=0, z=L), A(x)$ is the heat-transport area perpendicular to $x$, and $P(x)$ is the perimeter through which the heat flux $\mathrm{q}_{\mathrm{S}}\left(\mathrm{MW} / \mathrm{m}^{2}\right)$ flows. The trajectory for the $\mathrm{x}$-axis is indicated on Fig. A-2. For the purposes of this estimate, the surface heat flux, $q_{s}$ incident upon the diverted flux region is assumed independent of $x$, although it might be expected that $q_{s}$ would be less in the high-field $(x=0, L)$ region compared to the lowerfield $(x=L+R, 2 L+R)$ region. The model assumptions for the dependence of heat iransport area, $A(x)$, and perimeter, $P(x)$, on $x$ is depicted on Fig. A-3. Clearly, in addition to ignoring the $A(x)$ variations indicated by $E q \cdot(A-1)$, the linear connection between the $x=(0, L)$ rod-like region and the $x=(L+R$, $2 L+R)$ shell-like region ignores complex multi-dimensional effects, where the $x=(L, L+R)$ base region connects the rod to the shell.

The electron parallel-field thermal conductivity is given by

$$
k(W / k e V m)=\frac{9.8(10)^{14}}{\ln \Lambda} T^{5 / 2}
$$

The top of the spheromak is not considered in this analysis, with the boundaryvalue temperatures $T_{0} \equiv T(x=0,2 L+R)$ being assumed as representative of this region where the flux diverts strongly to the plasma-gun electrodes. Lastly, Eq. (A-5) admits no gradients across the sheath of diverted flux, with the surface heat $f l u x$ at any given value of $x$ appearing as effective volumetric heat source.

The heat flux $q_{s}$ is assumed uniform around the spheromak and dominated by fusion power rather than ohmic dissipation (i.e., a spheromak with reactor relevance). If $\mathrm{P}_{\mathrm{F}}\left(\mathrm{MW} / \mathrm{m}^{3}\right)$ is the total fusion power density within the plasma $\left(E_{N}=14.1-\mathrm{MeV}\right.$ neutrons and $E_{\alpha}=3.5-\mathrm{MeV}$ alpha particle), then 


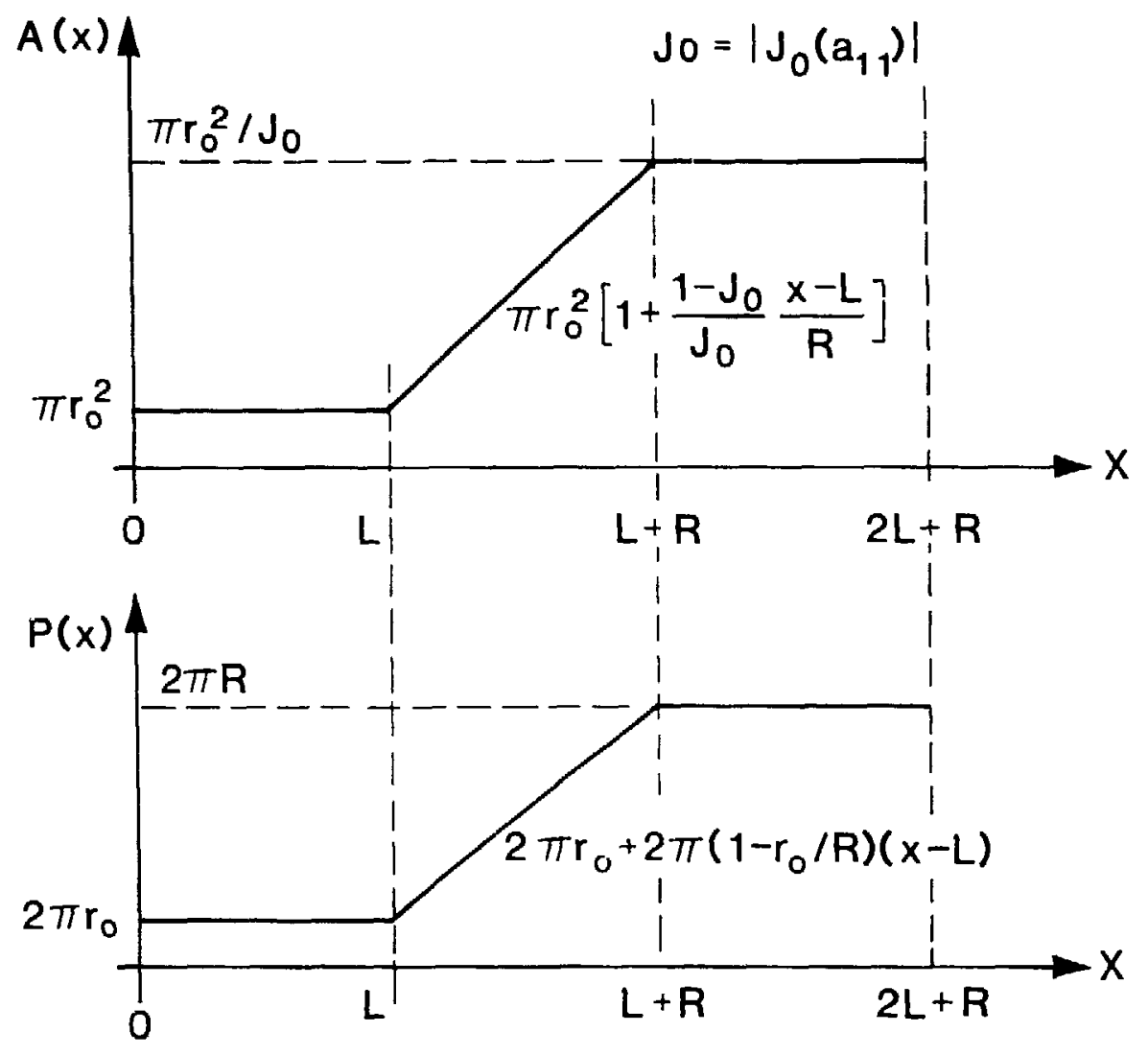

Fig. A-3. Model variation of heat-transport area, $A(x)$, as a function of the $x$-coordinate.

$$
q_{S}\left(M W / m^{2}\right)=\left(E_{\alpha} / E_{N}\right) P_{F} L \frac{R / L}{R / L+2 r_{0} / R+2} .
$$

Equation (A-5) is integrated to give for $\theta \equiv \mathrm{T} / \mathrm{T}_{\mathrm{o}}$ and $\rho \equiv \mathrm{R} / \mathrm{L}$

$$
\frac{2}{7} \frac{k_{0} T_{0}}{L q_{S}} \frac{p}{\left(R / r_{p}\right)}\left(\theta^{7 / 2}-1\right)=F(x)+20_{i} G(x),
$$

where $\rho$ is treated parametrically, $k_{0} \equiv k\left(T_{0}\right)$, and from Eq. (A-3), 
$R / r_{0}=2.4249 / \varepsilon^{1 / 2}$, with $\varepsilon$ also being treated parametrically. Given below are the functions $F(x), G(x)$, and $Q(x)$, with $J_{0} \equiv J_{0}\left(a_{11}\right)$.

$$
\begin{aligned}
& Q(x)=\int_{0}^{x} q_{s} P(x) d x \\
& G(x)=\frac{\pi r_{0}^{2}}{L} \int_{0}^{x} \frac{d x}{A(x)} \\
& F(x)=\frac{r_{0}}{L^{2} q_{S}} \int_{0}^{x} \frac{Q(x)}{A(x)} d x .
\end{aligned}
$$

The constant $Q_{i}$ is the total heat loss from the spheromak at $x=0$ and for $\theta(0,2 L+R)=1$ must equal $-F(2 L+R) / G(2 L+R)$. Hence, $Q_{i}=Q\left(x^{*}\right)$, where $x^{*}$ is the point along $x$ where $d T / d x=0$. With $y \equiv x / L, a \equiv\left(R / r_{0}-1\right) / \rho$, and $b \equiv\left(1-J_{0}\right) / J_{0} \rho=1.4829 / \rho$, the following expressions result for the functions $G(x), Q(x)$, and $F(x)$ :

$$
\begin{array}{rlrl}
G(x) & y & {[y=0 \rightarrow 1]} \\
=1+\rho \frac{J_{0}}{1-J_{0}} \log \left[1+\frac{1-J_{0}}{J_{0} \rho}(y-1)\right] & {[y=1 \rightarrow 1+\rho]} \\
=1+\frac{\rho J_{0}}{1-J_{0}} \log \left(1 / J_{0}\right)+J_{0}(y-1-\rho) & {[y=1+\rho \rightarrow 2+\rho]} \\
\frac{Q(x)}{2 \pi r_{0} L q_{S}}=y+\frac{1}{2} a(y-1)^{2} & {[y=0 \rightarrow 1]} \\
=1+\rho+\frac{1}{2} a \rho^{2}+\frac{R}{r_{0}}(y-1-\rho) & {[y=1+\rho \rightarrow 2+\rho]}
\end{array}
$$




$$
\begin{aligned}
=y^{2} & {[y=0 \rightarrow 1] } \\
=1 & +\frac{2\left(b^{2}-b+a / 2\right)}{b^{3}} \log [1+b(y-1)] \\
F(x) \quad & +(y-1) \frac{2 b-a}{b^{2}}+\frac{a}{2 b}(y-1)^{2} \quad[y=1 \rightarrow 1+\rho] \quad \text { (A-10C) } \\
=1 & +\frac{2\left(b^{2}-b+a / 2\right)}{b^{3}} \log [1+b \rho]+\frac{2 b-a}{b^{2}} \rho+\frac{1}{2} \frac{a}{b} \rho^{2} \\
& +J_{0}\left[2(1+\rho)\left(1-R / r_{0}\right)+a \rho^{2}\right][y-(1+\rho)] \\
& +\left(R / r_{0}\right) J_{0}\left[y^{2}-(1+\rho)^{2}\right] \quad[y=1+\rho \rightarrow 2+\rho] .
\end{aligned}
$$

The following expression results for the total heat delivered to the inner electrode.

$$
\begin{aligned}
& \frac{Q_{i}}{\pi r_{0} L q_{s}}\left[1+\frac{1}{b} \log \left(1 / J_{0}\right)+J_{0}\right]=1+\frac{2\left(b^{2}-b+a / 2\right)}{b^{3}} \log (1+b \rho) \\
& +\frac{(2 b-a) \rho}{b^{2}}+\frac{a}{2} \rho^{2}+J_{0}\left[2(1+\rho)\left(1-R / r_{o}\right)+a \rho^{2}+\left(R / r_{o}\right)(3+2 \rho)\right] .
\end{aligned}
$$

The total power deposited into the electrode/divertor system is given by

$$
\frac{Q_{T}}{\pi r_{0} L q_{S}}=2+(2+\rho) \frac{R}{r_{0}} \text {, }
$$

which allows the fraction, $f_{i}=Q_{i} / Q_{T}$, of the total power delivered to the inner electrode to be estimated as a function of $\varepsilon$ and $\rho=R / L$. Determinations of temperature profiles $[E q .(A-8)]$ and temperature peaking, $T\left(x^{*}\right) / T$, requires $P_{F}$, $L$, and $T_{0}$ to be specified. The following values are used to examine this variation: 


$$
\begin{aligned}
& \mathrm{p}_{\mathrm{F}}=100 \mathrm{MW} / \mathrm{m}^{3} \\
& \mathrm{~L}=2 \mathrm{~m} \\
& \mathrm{~T}_{\mathrm{O}}=0.05 \mathrm{keV} \\
& \mathrm{ln \Lambda}=10
\end{aligned}
$$

The parameters $1 / p$ and $\varepsilon$ are varied over the raiges $(0.5,2.0)$ and $(0.005$, $0.15)$, respectively.

The dependence of $\mathrm{F}_{i}$ gir $p$ and $\varepsilon$ is shown on Fig. A-4. Generally, less than $20 \%$ of the alpha-particle power is expected to impinge on the inner plasma-gun electrode, which, if $q_{S}$ is in fact less for the $x=0 \rightarrow L$ region because of the higher fields, may be even less. Hence, for nominally low radiation losses the outer plasma-gun electrode is expected to receive a majority of the fusion surface heat load. For the parameters assumed to obtain $T(x)$, modest temperature peaking in the scrape off is expected (Fig. A-4). This peaking occurs near the outer base of the spheromak and amounts to at most a factor of 2-3 when averaged around the plasmoid. The normalized temperature profiles (dependent only on $p$ and $\varepsilon$ ) are depicted on Fig. A-5. 


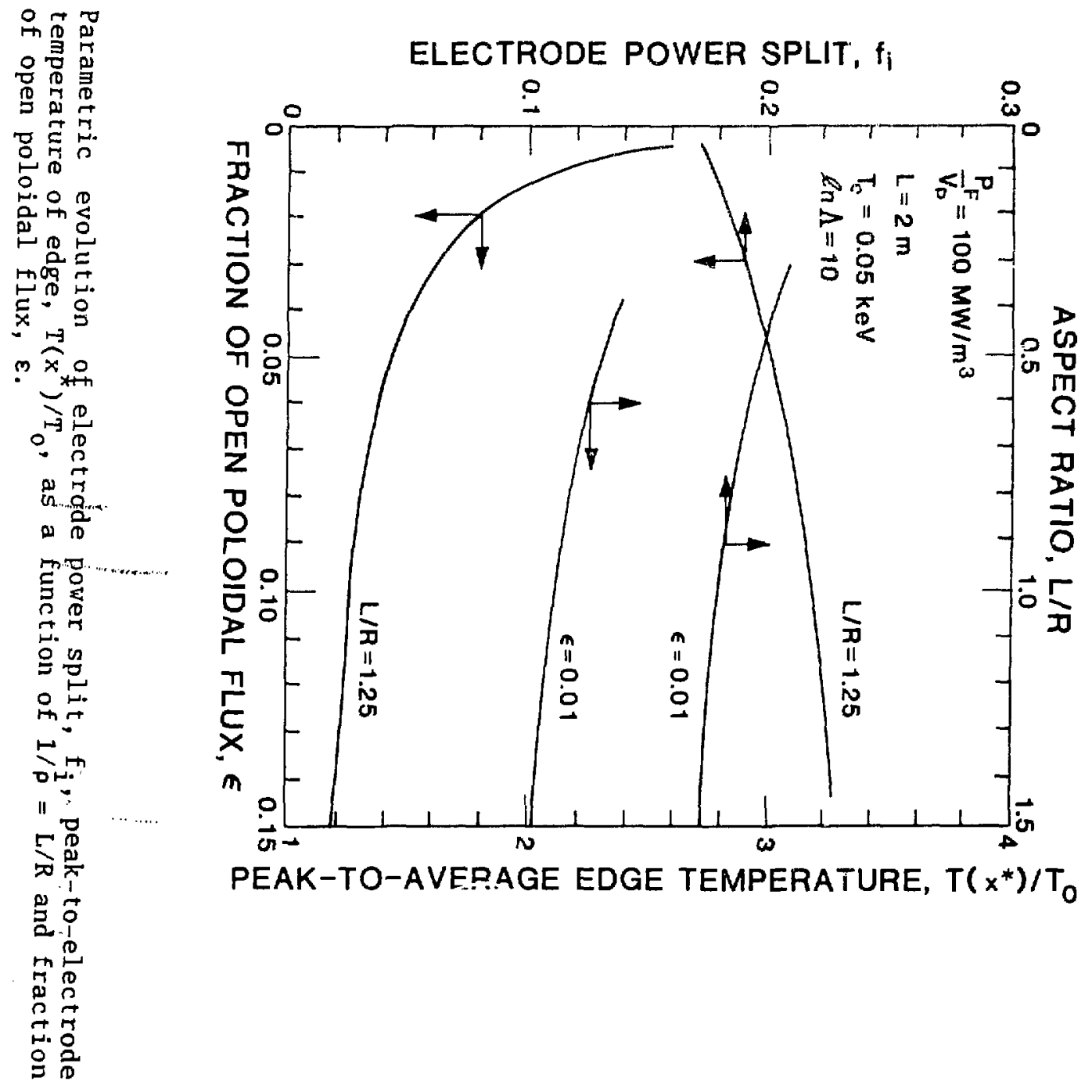




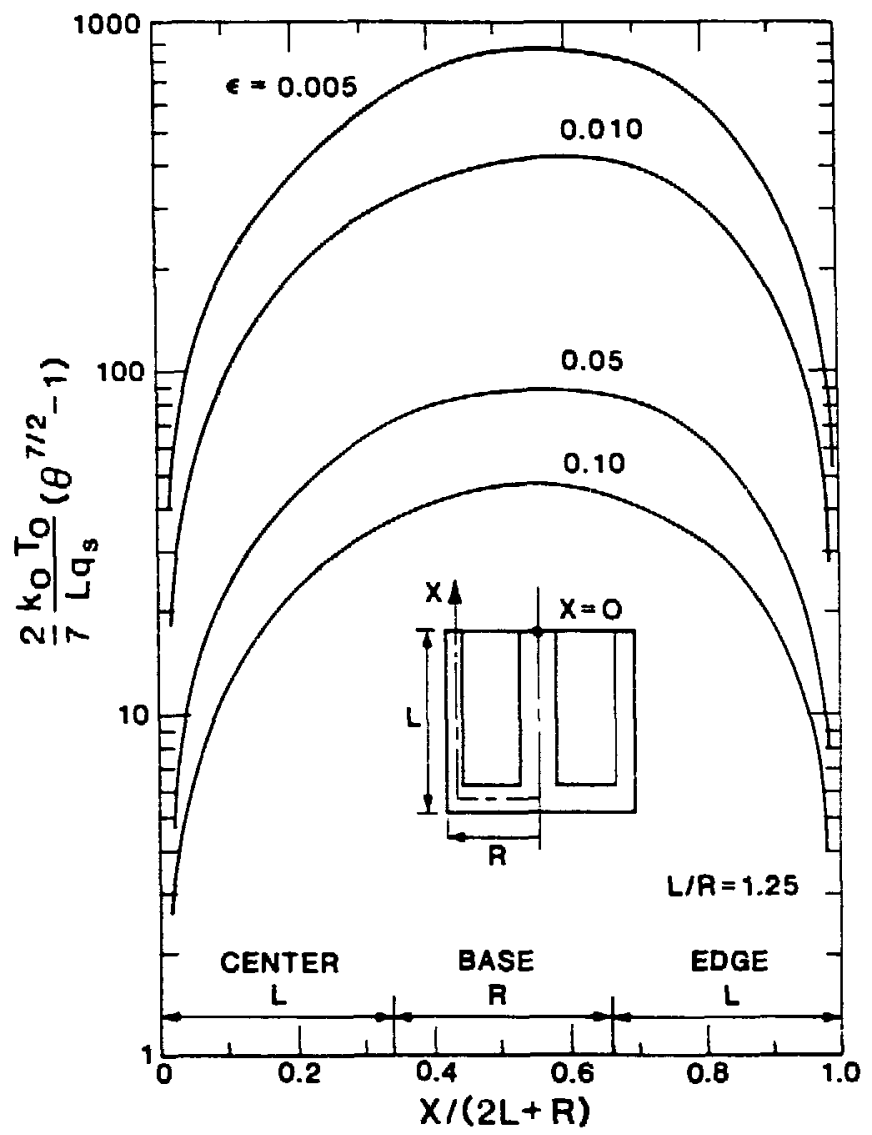

Fig. A-5. Normalized edge temperature profiles around the "cylindrical" spheromak, shows temperature peaking and the "water-shed" point occurring at a point near the outer "base" of the spheromak. 